\title{
Cellularity of free products of Boolean algebras (or topologies)
}

\author{
by
}

Saharon Shelah (Jerusalem and New Brunswick, NJ)

Abstract. The aim this paper is to present an answer to Problem 1 of Monk [10], [11]. We do this by proving in particular that if $\mu$ is a strong limit singular cardinal, $\theta=\left(2^{\operatorname{cf}(\mu)}\right)^{+}$and $2^{\mu}=\mu^{+}$then there are Boolean algebras $\mathbb{B}_{1}, \mathbb{B}_{2}$ such that

$$
\mathrm{c}\left(\mathbb{B}_{1}\right)=\mu, \quad \mathrm{c}\left(\mathbb{B}_{2}\right)<\theta \quad \text { but } \quad \mathrm{c}\left(\mathbb{B}_{1} * \mathbb{B}_{2}\right)=\mu^{+} .
$$

Further we improve this result, deal with the method and the necessity of the assumptions. In particular we prove that if $\mathbb{B}$ is a ccc Boolean algebra and $\mu^{\beth_{\omega}} \leq \lambda=\operatorname{cf}(\lambda) \leq 2^{\mu}$ then $\mathbb{B}$ satisfies the $\lambda$-Knaster condition (using the "revised GCH theorem").

\section{Introduction}

Notation 0.1. (1) In the present paper all cardinals are infinite so we will not repeat this additional demand. Cardinals will be denoted by $\lambda, \mu$, $\theta$ (with possible indices) while ordinal numbers will be called $\alpha, \beta, \zeta, \xi, \varepsilon$, $i, j$. Usually $\delta$ will stand for a limit ordinal (we may forget to repeat this assumption).

(2) Sequences of ordinals will be called $\eta, \nu, \varrho$ (with possible indices). For sequences $\eta_{1}, \eta_{2}$ their longest common initial segment is denoted by $\eta_{1} \wedge \eta_{2}$. The length of the sequence $\eta$ is $\lg (\eta)$.

(3) Ideals are supposed to be proper and contain all singletons. For a limit ordinal $\delta$ the ideal of bounded subsets of $\delta$ is denoted by $J_{\delta}^{\text {bd }}$. If $I$ is an ideal on a set $X$ then $I^{+}$is the family of $I$-large sets, i.e.

$$
a \in I^{+} \text {if and only if } a \subseteq X \& a \notin I,
$$

and $I^{\mathrm{c}}$ is the dual filter of sets with the complements in $I$.

2000 Mathematics Subject Classification: 54A25, 06E99, 03E04.

Key words and phrases: set theory, pcf, Boolean algebras, cellularity, product, colourings.

The research was partially supported by the Israel Science Foundation, founded by the Israel Academy of Sciences and Humanities. Publication 575. We thank Andrzej Rosłanowski for writing Sections 1-5 from lectures, and 6-7 from notes. 
Notation 0.2. (1) In a Boolean algebra we denote the Boolean operations by $\cap($ and $\bigcap), \cup$ (and $\cup$ ), - . The distinguished elements are $\mathbf{0}$ and $\mathbf{1}$. In the cases which may be confusing we will add indices to underline in which Boolean algebra the operation (or element) is considered, but generally we will not do it.

(2) For a Boolean algebra $\mathbb{B}$ and an element $x \in \mathbb{B}$ we write

$$
x^{0}=x \quad \text { and } \quad x^{1}=-x .
$$

(3) The free product of Boolean algebras $\mathbb{B}_{1}, \mathbb{B}_{2}$ is denoted by $\mathbb{B}_{1} * \mathbb{B}_{2}$. We will use $\star$ to denote the free product of a family of Boolean algebras.

Definition 0.3. (1) A Boolean algebra $\mathbb{B}$ satisfies the $\lambda$ - $c c$ if there is no family $\mathcal{F} \subseteq \mathbb{B}^{+}:=\mathbb{B} \backslash\{\mathbf{0}\}$ such that $|\mathcal{F}|=\lambda$ and any two members of $\mathcal{F}$ are disjoint (i.e., their meet in $\mathbb{B}$ is $\mathbf{0}$ ).

(2) The cellularity of the algebra $\mathbb{B}$ is

$$
\begin{aligned}
\mathrm{c}(\mathbb{B}) & =\sup \left\{|\mathcal{F}|: \mathcal{F} \subseteq \mathbb{B}^{+} \&(\forall x, y \in \mathcal{F})(x \neq y \Rightarrow x \cap y=\mathbf{0})\right\}, \\
\mathrm{c}^{+}(\mathbb{B}) & =\sup \left\{|\mathcal{F}|^{+}: \mathcal{F} \subseteq \mathbb{B}^{+} \&(\forall x, y \in \mathcal{F})(x \neq y \Rightarrow x \cap y=\mathbf{0})\right\} .
\end{aligned}
$$

(3) For a topological space $(X, \tau)$,

$\mathrm{c}(X, \tau)=\sup \{|\mathcal{U}|: \mathcal{U}$ is a family of pairwise disjoint

$$
\text { non-empty open sets\}. }
$$

The problem can be posed in each of the three ways $(\lambda-c c$ is the way of forcing, the cellularity of Boolean algebras is the approach of Boolean algebraists, and the cellularity of a topological space is the way of general topologists). It is well known that the three are equivalent, though (1) makes the attainment problem more explicit. We use the second approach.

A stronger property than $\lambda$-cc is the $\lambda$-Knaster property. This property behaves nicely in free products - it is productive. We will use it in our construction.

Definition 0.4. A Boolean algebra $\mathbb{B}$ has the $\lambda$-Knaster property if for every sequence $\left\langle z_{\varepsilon}: \varepsilon\langle\lambda\rangle \subseteq \mathbb{B}^{+}\right.$there is $A \in[\lambda]^{\lambda}$ such that

$$
\varepsilon_{1}, \varepsilon_{2} \in A \quad \Rightarrow \quad z_{\varepsilon_{1}} \cap z_{\varepsilon_{2}} \neq \mathbf{0} .
$$

We are interested in the behaviour of the cellularity of Boolean algebras when their free product is considered.

Thema 0.5. When, for Boolean algebras $\mathbb{B}_{1}, \mathbb{B}_{2}$,

$$
\mathrm{c}^{+}\left(\mathbb{B}_{1}\right) \leq \lambda_{1} \& \mathrm{c}^{+}\left(\mathbb{B}_{2}\right) \leq \lambda_{2} \quad \Rightarrow \quad \mathrm{c}^{+}\left(\mathbb{B}_{1} * \mathbb{B}_{2}\right) \leq \lambda_{1}+\lambda_{2} ?
$$

There are a lot of results about it, particularly if $\lambda_{1}=\lambda_{2}$ (see [22] or [10], more [24]). It is well known that if

$$
\left(\lambda_{1}^{+}+\lambda_{2}^{+}\right) \rightarrow\left(\lambda_{1}^{+}, \lambda_{2}^{+}\right)^{2}
$$


then the answer is "yes". These are exactly the cases for which the "yes" answer is known. Under GCH the only problem which remained open was the one presented below:

The Problem We Adddress 0.6 (posed by D. Monk as Problem 1 in [10], [11] under $\mathrm{GCH}$ ). Are there Boolean algebras $\mathbb{B}_{1}, \mathbb{B}_{2}$ and cardinals $\mu, \theta$ such that:

(1) $\lambda_{1}=\mu$ is singular, $\mu>\lambda_{2}=\theta>\operatorname{cf}(\mu)$ and

(2) $\mathrm{c}\left(\mathbb{B}_{1}\right)=\mu, \mathrm{c}\left(\mathbb{B}_{2}\right) \leq \theta$ but $\mathrm{c}\left(\mathbb{B}_{1} * \mathbb{B}_{2}\right)>\mu$ ?

We will answer this question proving in particular the following result (see 4.4):

- If $\mu$ is a strong limit singular cardinal, $\theta=\left(2^{\operatorname{cf}(\mu)}\right)^{+}$and $2^{\mu}=\mu^{+}$then there are Boolean algebras $\mathbb{B}_{1}, \mathbb{B}_{2}$ such that

$$
\mathrm{c}\left(\mathbb{B}_{1}\right)=\mu, \quad \mathrm{c}\left(\mathbb{B}_{2}\right)<\theta \quad \text { but } \quad \mathrm{c}\left(\mathbb{B}_{1} * \mathbb{B}_{2}\right)=\mu^{+} .
$$

Later we deal with better results by refining the method.

REMARK 0.7. On products of many Boolean algebras and square bracket arrows see $[17,1.2 \mathrm{~A}, 1.3 \mathrm{~B}]$.

If $\lambda \rightarrow[\mu]_{\theta}^{2}$, is the cardinal $\theta$ is possibly finite, $\mathbb{B}_{i}($ for $i<\theta)$ are Boolean algebras such that for each $j<\theta$ the free product $\star_{i \in \theta \backslash\{j\}} \mathbb{B}_{i}$ satisfies the $\mu$-cc then the algebra $\mathbb{B}=\star_{i<\theta} \mathbb{B}_{i}$ satisfies the $\lambda$-cc.

[Why? Assume $\left\langle a_{i}^{\zeta}: i<\theta\right\rangle \in \prod_{i<\theta} \mathbb{B}_{i}^{+}$(for $\zeta<\lambda$ ) such that for every $\zeta<\xi<\lambda$, for some $i=i(\zeta, \xi), \mathbb{B}_{i}=" a_{i}^{\zeta} \cap a_{i}^{\xi}=\mathbf{0}$ ". We can find $A \in[\lambda]^{\mu}$ and $i^{*}<\theta$ such that $i(\zeta, \xi) \neq i^{*}$ for $\zeta<\xi$ from $A$. Then $\left\langle a_{i}^{\zeta}: i<\theta, i \neq i^{*}\right\rangle$ for $\zeta \in A$ exemplifies that $\star_{i \in \theta \backslash\left\{i^{*}\right\}} \mathbb{B}_{i}$ fails the $\mu$-cc. We can also deal with ultraproducts and other products similarly.]

\section{Preliminaries: products of ideals}

Notation 1.1. For an ideal $J$ on $\delta$ the quantifier $\left(\forall^{J} i<\delta\right)$ means "for all $i<\delta$ except a set from the ideal $J$ ", i.e.,

$$
\left(\forall^{J} i<\delta\right) \varphi(i) \equiv\{i<\delta: \neg \varphi(i)\} \in J .
$$

The dual quantifier $\left(\exists^{J} i<\delta\right)$ means "for a $J$-positive set of $i<\delta^{\text {". }}$.

Proposition 1.2. Assume that $\lambda^{0}>\lambda^{1}>\ldots>\lambda^{n-1}$ are cardinals, $I^{l}$ are ideals on $\lambda^{l}($ for $l<n)$ and $B \subseteq \prod_{l<n} \lambda^{l}$. Further suppose that:

$(\alpha)\left(\exists^{0} \gamma_{0}\right) \ldots\left(\exists^{I^{n-1}} \gamma_{n-1}\right)\left(\left\langle\gamma_{l}: l<n\right\rangle \in B\right)$,

$(\beta)$ the ideal $I^{l}$ is $\left(2^{\lambda^{l+1}}\right)^{+}$-complete $($for $l+1<n)$.

Then there are sets $X_{l} \subseteq \lambda^{l}, X_{l} \notin I^{l}$ such that $\prod_{l<n} X_{l} \subseteq B$. 
[Note that this translates the situation to arity 1; it is a kind of polarized $(1, \ldots, 1)$-partition with ideals.]

Proof. We show it by induction on $n$. Define

$$
\begin{aligned}
E_{0}:=\left\{\left(\gamma^{\prime}, \gamma^{\prime \prime}\right):\right. & \gamma^{\prime}, \gamma^{\prime \prime}<\lambda^{0} \text { and for all } \gamma_{1}<\lambda^{1}, \ldots, \gamma_{n-1}<\lambda^{n-1}, \\
& \left.\left(\left\langle\gamma^{\prime}, \gamma_{1}, \ldots, \gamma_{n-1}\right\rangle \in B \Leftrightarrow\left\langle\gamma^{\prime \prime}, \gamma_{1}, \ldots, \gamma_{n-1}\right\rangle \in B\right)\right\} .
\end{aligned}
$$

Clearly $E_{0}$ is an equivalence relation on $\lambda^{0}$ with $\leq 2^{\prod_{0<m<n} \lambda^{m}}=2^{\lambda^{1}}$ equivalence classes. Hence the set

$$
A_{0}:=\bigcup\left\{A: A \text { is an } E_{0} \text {-equivalence class, } A \in I^{0}\right\}
$$

is in the ideal $I^{0}$. Let

$$
A_{0}^{*}:=\left\{\gamma_{0}<\lambda^{0}:\left(\exists^{I^{1}} \gamma_{1}\right) \ldots\left(\exists^{I^{n-1}} \gamma_{n-1}\right)\left(\left\langle\gamma_{0}, \gamma_{1}, \ldots, \gamma_{n-1}\right\rangle \in B\right)\right\} .
$$

The assumption $(\alpha)$ implies that $A_{0}^{*} \notin I^{0}$ and hence we may choose $\gamma_{0}^{*} \in$ $A_{0}^{*} \backslash A_{0}$. Let

$$
B_{1}:=\left\{\bar{\gamma} \in \prod_{k=1}^{n-1} \lambda^{k}:\left\langle\gamma_{0}^{*}\right\rangle\ulcorner\bar{\gamma} \in B\} .\right.
$$

Since $\gamma_{0}^{*} \in A_{0}^{*}$ we are sure that

$$
\left(\exists^{I^{1}} \gamma_{1}\right) \ldots\left(\exists^{I^{n-1}} \gamma_{n-1}\right)\left(\left\langle\gamma_{1}, \ldots, \gamma_{n-1}\right\rangle \in B_{1}\right) .
$$

Hence we may apply the inductive hypothesis for $n-1$ and $B_{1}$ to find sets $X_{1} \in\left(I^{1}\right)^{+}, \ldots, X_{n-1} \in\left(I^{n-1}\right)^{+}$such that $\prod_{l=1}^{n-1} X_{l} \subseteq B_{1}$, so then

$$
\left(\forall \gamma_{1} \in X_{1}\right) \ldots\left(\forall \gamma_{n-1} \in X_{n-1}\right)\left(\left\langle\gamma_{0}^{*}, \gamma_{1}, \ldots, \gamma_{n-1}\right\rangle \in B\right) .
$$

Take $X_{0}$ to be the $E_{0}$-equivalence class of $\gamma_{0}^{*}$ (so $X_{0} \in\left(I^{0}\right)^{+}$as $\gamma_{0}^{*} \notin A_{0}$ ). By the definition of the relation $E_{0}$ and the choice of the sets $X_{l}$ we see that for each $\gamma_{0} \in X_{0}$,

$$
\left(\forall \gamma_{1} \in X_{1}\right) \ldots\left(\forall \gamma_{n-1} \in X_{n-1}\right)\left(\left\langle\gamma_{0}, \gamma_{1}, \ldots, \gamma_{n-1}\right\rangle \in B\right),
$$

which means that $\prod_{l<n} X_{l} \subseteq B$.

Proposition 1.3. Assume that $\lambda_{0}>\lambda_{1}>\ldots>\lambda_{n-1} \geq \sigma$ are cardinals, $I_{l}$ are ideals on $\lambda_{l}($ for $l<n)$ and $B \subseteq \prod_{l<n} \lambda_{l}$. Further suppose that:

$(\alpha)\left(\exists^{I_{0}} \gamma_{0}\right) \ldots\left(\exists^{I_{n-1}} \gamma_{n-1}\right)\left(\left\langle\gamma_{l}: l<n\right\rangle \in B\right)$,

( $\beta$ ) $I_{l}$ is $\left(\left(\lambda_{l+1}\right)^{\sigma}\right)^{+}$-complete for each $l<n-1$, and $\left[\lambda_{n-1}\right]^{<\sigma} \subseteq I_{n-1}$.

Then there are sets $X_{l} \in\left[\lambda_{l}\right]^{\sigma}$ such that $\prod_{l<n} X_{l} \subseteq B$.

Proof. The proof is by induction on $n$. If $n=1$ then there is nothing to do as $I_{n-1}$ contains all subsets of $\lambda_{n-1}$ of size $<\sigma$ and $\lambda_{n_{1}} \geq \sigma$ so every $A \in I_{n_{1}}^{+}$has cardinality $\geq \sigma$.

Let $n>1$ and let

$$
a_{0}:=\left\{\gamma \in \lambda_{0}:\left(\exists^{I_{1}} \gamma_{1}\right) \ldots\left(\exists^{I_{n-1}} \gamma_{n-1}\right)\left(\left\langle\gamma, \gamma_{1}, \ldots, \gamma_{n-1}\right\rangle \in B\right)\right\}
$$


By our assumptions we know that $a_{0} \in\left(I_{0}\right)^{+}$. For each $\gamma \in a_{0}$ we may apply the inductive hypothesis to the set

$$
B_{\gamma}:=\left\{\left\langle\gamma_{1}, \ldots, \gamma_{n-1}\right\rangle \in \prod_{0<l<n} \lambda_{l}:\left\langle\gamma, \gamma_{1}, \ldots, \gamma_{n-1}\right\rangle \in B\right\}
$$

and get sets $X_{1}^{\gamma} \in\left[\lambda_{1}\right]^{\sigma}, \ldots, X_{n-1}^{\gamma} \in\left[\lambda_{n-1}\right]^{\sigma}$ such that

$$
\prod_{0<l<n} X_{l}^{\gamma} \subseteq B_{\gamma}
$$

There are at most $\left(\lambda_{1}\right)^{\sigma}$ possible sequences $\left\langle X_{1}^{\gamma}, \ldots, X_{n-1}^{\gamma}\right\rangle$, and the ideal $I_{0}$ is $\left(\left(\lambda_{1}\right)^{\sigma}\right)^{+}$-complete, so for some sequence $\left\langle X_{1}, \ldots, X_{n-1}\right\rangle$ and a set $a^{*} \subseteq a_{0}, a^{*} \in\left(I_{0}\right)^{+}$we have

$$
\left(\forall \gamma \in a^{*}\right)\left(X_{1}^{\gamma}=X_{1} \& \ldots \& X_{n-1}^{\gamma}=X_{n-1}\right) .
$$

Choose $X_{0} \in\left[a^{*}\right]^{\sigma}$ (remember that $I_{0}$ contains singletons and it is complete enough to make sure that $\left.\sigma \leq\left|a^{*}\right|\right)$. Clearly $\prod_{l<n} X_{l} \subseteq B$.

REMARK 1.4. We can use $\sigma_{0} \geq \sigma_{1} \geq \ldots \geq \sigma_{n-1}, I_{l}$ is $\left(\lambda_{l+1}^{\sigma_{l+1}}\right)^{+}$complete, $\left[\lambda_{l}\right]^{<\sigma_{l}} \subseteq I_{l}$.

Proposition 1.5. Assume that $n<\omega$ and $\lambda_{l}^{m}, \chi_{l}^{m}, P_{l}^{m}, I_{l}^{m}, I^{m}$ and $B$ are such that for $l, m \leq n$ :

( $\alpha$ ) $I_{l}^{m}$ is a $\chi_{l}^{m}$-complete ideal on $\lambda_{l}^{m}($ for $l, m \leq n)$,

( $\beta) P_{l}^{m} \subseteq \mathcal{P}\left(\lambda_{l}^{m}\right)$ is a family dense in $\left(I_{l}^{m}\right)^{+}$in the sense that

$$
\left(\forall X \in\left(I_{l}^{m}\right)^{+}\right)\left(\exists a \in P_{l}^{m}\right)(a \subseteq X),
$$

$(\gamma) I^{m}=\left\{X \subseteq \prod_{l<n} \lambda_{l}^{m}: \neg\left(\exists^{I_{0}^{m}} \gamma_{0}\right) \ldots\left(\exists^{I_{n}^{m}} \gamma_{n}\right)\left(\left\langle\gamma_{0}, \ldots, \gamma_{n}\right\rangle \in X\right)\right\}[$ thus $I^{m}$ is the ideal on $\prod_{l \leq n} \lambda_{l}^{m}$ such that the dual filter $\left(I^{m}\right)^{\mathrm{c}}$ is the Fubini product of the filters $\left.\left(I_{0}^{m}\right)^{\mathrm{c}}, \ldots,\left(I_{n}^{m}\right)^{\mathrm{c}}\right]$,

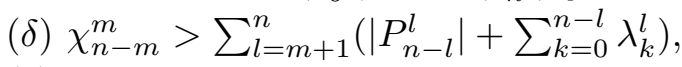

( $\varepsilon) B \subseteq \prod_{m \leq n} \prod_{l \leq n} \lambda_{l}^{m}$ is a set satisfying

$$
\left(\exists^{I^{0}} \eta_{0}\right)\left(\exists^{I^{1}} \eta_{1}\right) \ldots\left(\exists^{I^{n}} \eta_{n}\right)\left(\left\langle\eta_{0}, \eta_{1}, \ldots, \eta_{n}\right\rangle \in B\right) .
$$

Then there are sets $X_{0}, \ldots, X_{n}$ such that for $m \leq n$ :

(a) $X_{m} \subseteq \prod_{l \leq n-m} \lambda_{l}^{m}$

(b) if $\eta, \nu \in X_{m}, \eta \neq \nu$ then

(i) $\eta \uparrow(n-m)=\nu \uparrow(n-m)$,

(ii) $\eta(n-m) \neq \nu(n-m)$

(c) $\left\{\eta(n-m): \eta \in X_{m}\right\} \in P_{n-m}^{m}$,

(d) for each $\left\langle\eta_{0}, \ldots, \eta_{n}\right\rangle \in \prod_{m \leq n} X_{m}$ there is $\left\langle\eta_{0}^{*}, \ldots, \eta_{n}^{*}\right\rangle \in B$ such that $(\forall m \leq n)\left(\eta_{m} \unlhd \eta_{m}^{*}\right)$. 
REMARK 1.5.A. (1) Note that the sets $X_{m}$ in the assertion of 1.5 may be thought of as sets of the form $X_{m}=\left\{\nu_{m} \frown\langle\alpha\rangle: \alpha \in a_{m}\right\}$ for some $\nu_{m} \in \prod_{l<n-m} \lambda_{l}^{m}$ and $a_{m} \in P_{n-m}^{m}$.

(2) We will apply this proposition with $\lambda_{l}^{m}=\lambda_{l}, I_{l}^{m}=I_{l}$ and $\lambda_{l}>\chi_{l}>$ $\sum_{k<l} \lambda_{k}$.

(3) In the assumption $(\delta)$ of 1.5 we may assume that the last sum on the right hand side ranges from $k=0$ to $n-l-1$. We did not formulate that assumption in this way as with $n-l$ it is easier to handle the induction step and this change is not important for our applications.

(4) In the assertion (d) of 1.5 we can have $\eta_{l}^{*}$ depending on $\left\langle\eta_{0}, \ldots, \eta_{l}\right\rangle$ only.

Proof (of Proposition 1.5). The proof is by induction on $n$. For $n=0$ there is nothing to do. Let us describe the induction step.

Suppose $0<n<\omega$ and $\lambda_{l}^{m}, \chi_{l}^{m}, P_{l}^{m}, I_{l}^{m}, I^{m}$ (for $l, m \leq n$ ) and $B$ satisfy the assumptions $(\alpha)-(\varepsilon)$. Let

$$
\begin{aligned}
B^{*}:=\left\{\left\langle\eta_{0}, \eta_{1}\left\lceil n, \ldots, \eta_{n}\lceil n\rangle: \eta_{m} \in \prod_{l \leq n} \lambda_{l}^{m}(\text { for } m \leq n)\right. \text { and }\right.\right. \\
\\
\left.\qquad\left\langle\eta_{0}, \eta_{1}, \ldots, \eta_{n}\right\rangle \in B\right\},
\end{aligned}
$$

and for $\eta_{0} \in \prod_{l \leq n} \lambda_{l}^{0}$ let

$$
B_{\eta_{0}}^{*}:=\left\{\left\langle\nu_{1}, \ldots, \nu_{n}\right\rangle \in \prod_{m=1}^{n} \prod_{l=0}^{n-1} \lambda_{l}^{m}:\left\langle\eta_{0}, \nu_{1}, \ldots, \nu_{n}\right\rangle \in B^{*}\right\} .
$$

Let $J^{m}$ (for $1 \leq m \leq n$ ) be the ideal on $\prod_{l=0}^{n-1} \lambda_{l}^{m}$ coming from the ideals $I_{l}^{m}$, i.e., a set $X \subseteq \prod_{l<n} \lambda_{l}^{m}$ is in $J^{m}$ if and only if

$$
\neg\left(\exists^{I_{0}^{m}} \gamma_{0}\right) \ldots\left(\exists^{I_{n-1}^{m}} \gamma_{n-1}\right)\left(\left\langle\gamma_{0}, \ldots, \gamma_{n-1}\right\rangle \in X\right) .
$$

Let us call the set $B_{\eta_{0}}^{*}$ big if

$$
\left(\exists^{J^{1}} \nu_{1}\right) \ldots\left(\exists^{J^{n}} \nu_{n}\right)\left(\left\langle\nu_{1}, \ldots, \nu_{n}\right\rangle \in B_{\eta_{0}}^{*}\right) .
$$

We may write more explicitly what the bigness means: the above condition is equivalent to

$$
\begin{aligned}
&\left(\exists^{I_{0}^{1}} \gamma_{0}^{1}\right) \ldots\left(\exists^{I_{n-1}^{1}} \gamma_{n-1}^{1}\right) \ldots \\
& \ldots\left(\exists^{I_{0}^{n}} \gamma_{0}^{n}\right) \ldots\left(\exists^{I_{n-1}^{n}} \gamma_{n-1}^{n}\right)\left(\left\langle\left\langle\gamma_{0}^{1}, \ldots, \gamma_{n-1}^{1}\right\rangle, \ldots\left\langle\gamma_{0}^{n}, \ldots, \gamma_{n-1}^{n}\right\rangle\right\rangle \in B_{\eta_{0}}^{*}\right),
\end{aligned}
$$

which means

$$
\begin{aligned}
& \left(\exists I_{0}^{1} \gamma_{0}^{1}\right) \ldots \ldots\left(\exists^{I_{n-1}^{n}} \gamma_{n-1}^{n}\right) \\
& \left(\exists \gamma_{n}^{1}\right) \ldots\left(\exists \gamma_{n}^{n}\right)\left(\left\langle\eta_{0},\left\langle\gamma_{0}^{1}, \ldots, \gamma_{n}^{1}\right\rangle, \ldots,\left\langle\gamma_{0}^{n}, \ldots, \gamma_{n}^{n}\right\rangle\right\rangle \in B\right) .
\end{aligned}
$$

By the assumptions $(\gamma)$ and $(\varepsilon)$ we know that

$$
\begin{aligned}
&\left(\exists^{I_{0}^{0}} \gamma_{0}^{0}\right) \ldots\left(\exists^{I_{n}^{0}} \gamma_{n}^{0}\right)\left(\exists^{I_{0}^{1}} \gamma_{0}^{1}\right) \ldots\left(\exists^{I_{n}^{1}} \gamma_{n}^{1}\right) \ldots \\
& \ldots\left(\exists^{I_{0}^{n}} \gamma_{0}^{n}\right) \ldots\left(\exists^{I_{n}^{n}} \gamma_{n}^{n}\right)\left(\left\langle\left\langle\gamma_{0}^{0}, \ldots, \gamma_{n}^{0}\right\rangle,\left\langle\gamma_{0}^{1}, \ldots, \gamma_{n}^{1}\right\rangle, \ldots,\left\langle\gamma_{0}^{n}, \ldots, \gamma_{n}^{n}\right\rangle\right\rangle \in B\right) .
\end{aligned}
$$


Obviously any quantifier $\left(\exists_{l}^{m} \gamma_{l}^{m}\right)$ above may be replaced by $\left(\exists \gamma_{l}^{m}\right)$ and then "moved" right as for as we want. Consequently, we get

$$
\begin{aligned}
& \left(\exists \gamma_{0}^{0}\right) \ldots\left(\exists \gamma_{n-1}^{0}\right)\left(\exists^{I_{n}^{0}} \gamma_{n}^{0}\right)\left(\exists^{I_{0}^{1}} \gamma_{0}^{1}\right) \ldots\left(\exists^{I_{n-1}^{1}} \gamma_{n-1}^{1}\right) \ldots\left(\exists^{I_{0}^{n}} \gamma_{0}^{n}\right) \ldots\left(\exists^{I_{n-1}^{n}} \gamma_{n-1}^{n}\right) \\
& \left(\exists \gamma_{n}^{1}\right) \ldots\left(\exists \gamma_{n}^{n}\right)\left(\left\langle\left\langle\gamma_{0}^{0}, \ldots, \gamma_{n}^{0}\right\rangle,\left\langle\gamma_{0}^{1}, \ldots, \gamma_{n}^{1}\right\rangle, \ldots,\left\langle\gamma_{0}^{n}, \ldots, \gamma_{n}^{n}\right\rangle\right\rangle \in B\right),
\end{aligned}
$$

which means that

$$
\left(\exists \gamma_{0}^{0}\right) \ldots\left(\exists \gamma_{n-1}^{0}\right)\left(\exists^{I_{n}^{0}} \gamma_{n}^{0}\right)\left(B_{\left\langle\gamma_{0}^{0}, \ldots, \gamma_{n}^{n}\right\rangle}^{*} \text { is big }\right) .
$$

Hence we find $\gamma_{0}^{0}, \ldots, \gamma_{n-1}^{0}$ and a set $a \in\left(I_{n}^{0}\right)^{+}$such that

$$
(\forall \gamma \in a)\left(B_{\left\langle\gamma_{0}^{0}, \ldots, \gamma_{n}^{n}\right\rangle}^{*} \text { is big }\right) \text {. }
$$

Note that the assumptions of the proposition are such that if we know that $B_{\eta_{0}}^{*}$ is big then we may apply the inductive hypothesis to $\lambda_{l}^{m}, \chi_{l}^{m}, P_{l}^{m}$, $I_{l}^{m}, J^{m}$ (for $1 \leq m \leq n, l \leq n-1$ ) and $B_{\eta_{0}}^{*}$. Consequently, for each $\gamma \in a$ we find sets $X_{1}^{\gamma}, \ldots, X_{n}^{\gamma}$ such that for $1 \leq m \leq n$ :

(a) ${ }^{*} X_{m}^{\gamma} \subseteq \prod_{l \leq n-m} \lambda_{l}^{m}$,

(b)* if $\eta, \nu \in X_{m}^{\gamma}, \eta \neq \nu$ then

(i) $\eta \uparrow(n-m)=\nu \uparrow(n-m)$, and

(ii) $\eta(n-m) \neq \nu(n-m)$,

(c)* $\left\{\eta(n-m): \eta \in X_{m}^{\gamma}\right\} \in P_{n-m}^{m}$,

(d)* for all $\left\langle\eta_{0}, \ldots, \eta_{n}\right\rangle \in \prod_{m \leq n} X_{m}^{\gamma}$ we have

$$
\left(\exists\left\langle\eta_{0}^{*}, \ldots, \eta_{n}^{*}\right\rangle \in B_{\left\langle\gamma_{0}^{0}, \ldots, \gamma_{n-1}^{0}, \gamma\right\rangle}^{*}\right)(\forall 1 \leq m \leq n)\left(\nu_{m} \unlhd \nu_{m}^{*}\right) .
$$

Now we may ask how many possibilities for $X_{m}^{\gamma}$ we have: not too many. If we fix the common initial segment (see $(b)^{*}$ ) the only freedom we have is in choosing an element of $P_{n-m}^{m}$ (see $\left.(\mathrm{c})^{*}\right)$. Consequently, there are at most $\left|P_{n-m}^{m}\right|+\sum_{l \leq n-m} \lambda_{l}^{m}$ possible values for $X_{m}^{\gamma}$ and hence there are at most

$$
\sum_{m=1}^{n}\left(\left|P_{n-m}^{m}\right|+\sum_{l \leq n-m} \lambda_{l}^{m}\right)<\chi_{n}^{0}
$$

possible values for the sequence $\left\langle X_{1}^{\gamma}, \ldots, X_{n}^{\gamma}\right\rangle$. Since the ideal $I_{n}^{0}$ is $\chi_{n^{-}}^{0}$ complete we find $\left\langle X_{1}, \ldots, X_{n}\right\rangle$ and a set $b \subseteq a, b \in\left(I_{n}^{0}\right)^{+}$, such that

$$
(\forall \gamma \in b)\left(\left\langle X_{1}^{\gamma}, \ldots, X_{n}^{\gamma}\right\rangle=\left\langle X_{1}, \ldots, X_{n}\right\rangle\right) .
$$

Next choose $b_{n}^{0} \in P_{n}^{0}$ such that $b_{n}^{0} \subseteq b$ and put

$$
X_{0}=\left\{\left\langle\gamma_{0}^{0}, \ldots, \gamma_{n-1}^{0}, \gamma\right\rangle: \gamma \in b_{n}^{0}\right\} .
$$

Now it is a routine to check that the sets $X_{0}, X_{1}, \ldots, X_{n}$ are as required (i.e., they satisfy clauses $(\mathrm{a})-(\mathrm{d}))$. 


\section{Cofinal sequences in trees}

Notation 2.1. For a tree $T \subseteq{ }^{\delta>} \mu$ the set of $\delta$-branches through $T$ is

$$
\lim _{\delta}(T):=\left\{\eta \in{ }^{\delta} \mu:(\forall \alpha<\delta)(\eta\lceil\alpha \in T)\} .\right.
$$

The $i$ th level (for $i<\delta$ ) of the tree $T$ is

$$
T_{i}:=T \cap{ }^{i} \mu
$$

and $T_{<i}:=\bigcup_{j<i} T_{j}$.

If $\eta \in T$ then the set of immediate successors of $\eta$ in $T$ is

$$
\operatorname{succ}_{T}:=\{\nu \in T: \eta \triangleleft \nu \& \lg (\nu)=\lg (\eta)+1\} .
$$

We shall not distinguish strictly between $\operatorname{succ}_{T}(\eta)$ and $\{\alpha: \eta \curlyvee\langle\alpha\rangle \in T\}$.

Definition 2.2. (1) $\mathcal{K}_{\mu, \delta}$ is the family of all pairs $(T, \bar{\lambda})$ such that $T \subseteq{ }^{\delta>} \mu$ is a tree with $\delta$ levels and $\bar{\lambda}=\left\langle\lambda_{\eta}: \eta \in T\right\rangle$ is a sequence of cardinals such that for each $\eta \in T$ we have $\operatorname{succ}_{T}(\eta)=\lambda_{\eta}$ (compare the previous remark about not distinguishing $\operatorname{succ}_{T}(\eta)$ and $\{\alpha: \eta\ulcorner\langle\alpha\rangle \in T\})$.

(2) For a limit ordinal $\delta$ and a cardinal $\mu$ we let

$$
\begin{aligned}
\mathcal{K}_{\mu, \delta}^{\mathrm{id}}:=\left\{(T, \bar{\lambda}, \bar{I}):(T, \bar{\lambda}) \in \mathcal{K}_{\mu, \delta}, \bar{I}=\left\langle I_{\eta}: \eta \in T\right\rangle,\right. & \\
& \text { each } \left.I_{\eta} \text { is an ideal on } \lambda_{\eta}=\operatorname{succ}_{T}(\eta)\right\} .
\end{aligned}
$$

Let $(T, \bar{\lambda}, \bar{I}) \in \mathcal{K}_{\mu, \delta}^{\text {id }}$ and let $J$ be an ideal on $\delta$ (including $J_{\delta}^{\text {bd }}$ if we do not say otherwise). Further let $\bar{\eta}=\left\langle\eta_{\alpha}: \alpha<\lambda\right\rangle \subseteq \lim _{\delta}(T)$ be a sequence of $\delta$-branches through $T$.

(3) We say that $\bar{\eta}$ is $J$-cofinal in $(T, \bar{\lambda}, \bar{I})$ if

(a) $\eta_{\alpha} \neq \eta_{\beta}$ for distinct $\alpha, \beta<\lambda$,

(b) for every sequence $\bar{A}=\left\langle A_{\eta}: \eta \in T\right\rangle \in \prod_{\eta \in T} I_{\eta}$ there is $\alpha^{*}<\lambda$ such that

$$
\alpha^{*} \leq \alpha<\lambda \quad \Rightarrow \quad\left(\forall^{J} i<\delta\right)\left(\eta_{\alpha}\left\lceil(i+1) \notin A_{\eta_{\alpha}\lceil i}\right) .\right.
$$

(4) If $I$ is an ideal on $\lambda$ then we say that $(\bar{\eta}, I)$ is a $J$-cofinal pair for $(T, \bar{\lambda}, \bar{I})$ if

(a) $\eta_{\alpha} \neq \eta_{\beta}$ for distinct $\alpha, \beta<\lambda$,

(b) for every sequence $\bar{A}=\left\langle A_{\eta}: \eta \in T\right\rangle \in \prod_{\eta \in T} I_{\eta}$ there is $A \in I$ such that

$$
\alpha \in \lambda \backslash A \quad \Rightarrow \quad\left(\forall^{J} i<\delta\right)\left(\eta_{\alpha} \uparrow(i+1) \notin A_{\eta_{\alpha} \backslash i}\right) .
$$

(5) The sequence $\bar{\eta}$ is strongly $J$-cofinal in $(T, \bar{\lambda}, \bar{I})$ if

(a) $\eta_{\alpha} \neq \eta_{\beta}$ for distinct $\alpha, \beta<\lambda$,

(b) for every $n<\omega$ and functions $F_{0}, \ldots, F_{n}$ there is $\alpha^{*}<\lambda$ such that if $m \leq n, \alpha_{0}<\ldots<\alpha_{n}<\lambda, \alpha^{*} \leq \alpha_{m}$ then the set of $i<\delta$ such that: 
(i) $(\forall l<m)\left(\lambda_{\eta_{\alpha_{l}} \uparrow i}<\lambda_{\eta_{\alpha_{m}} \uparrow i}\right)$ and

(ii) $F_{m}\left(\eta_{\alpha_{0}} \uparrow(i+1), \ldots, \eta_{\alpha_{m-1}} \uparrow(i+1), \eta_{\alpha_{m}}\left\lceil i, \ldots, \eta_{\alpha_{n}} \uparrow i\right) \in I_{\eta_{\alpha_{m}} \uparrow i}\right.$ (and well defined) but

$\eta_{\alpha_{m}}\left\lceil(i+1) \in F_{m}\left(\eta_{\alpha_{0}} \uparrow(i+1), \ldots, \eta_{\alpha_{m-1}} \uparrow(i+1), \eta_{\alpha_{m}}\left\lceil i, \ldots, \eta_{\alpha_{n}} \uparrow i\right)\right.\right.$,

is in the ideal $J$.

[Note: in (b) above we may have $\alpha^{*}<\alpha_{0}$, this causes no real change.]

(6) The sequence $\bar{\eta}$ is stronger $J$-cofinal in $(T, \bar{\lambda}, \bar{I})$ if

(a) $\eta_{\alpha} \neq \eta_{\beta}$ for distinct $\alpha, \beta<\lambda$,

(b) for every $n<\omega$ and functions $F_{0}, \ldots, F_{n}$ there is $\alpha^{*}<\lambda$ such that if $m \leq n, \alpha_{0}<\ldots<\alpha_{n}<\lambda, \alpha^{*} \leq \alpha_{m}$ then the set of $i<\delta$ such that:

(ii) $F_{m}\left(\eta_{\alpha_{0}} \uparrow(i+1), \ldots, \eta_{\alpha_{m-1}} \uparrow(i+1), \eta_{\alpha_{m}}\left\lceil i, \ldots, \eta_{\alpha_{n}}\lceil i) \in I_{\eta_{\alpha_{m}} \uparrow i}\right.\right.$ (and well defined) but

$\eta_{\alpha_{m}} \uparrow(i+1) \in F_{m}\left(\eta_{\alpha_{0}} \uparrow(i+1), \ldots, \eta_{\alpha_{m-1}} \uparrow(i+1), \eta_{\alpha_{m}}\left\lceil i, \ldots, \eta_{\alpha_{n}} \uparrow i\right)\right.$,

is in the ideal $J$.

(7) The sequence $\bar{\eta}$ is strongest $J$-cofinal in $(T, \bar{\lambda}, \bar{I})$ if

(a) $\eta_{\alpha} \neq \eta_{\beta}$ for distinct $\alpha, \beta<\lambda$,

(b) for every $n<\omega$ and functions $F_{0}, \ldots, F_{n}$ there is $\alpha^{*}<\lambda$ such that if $m \leq n, \alpha_{0}<\ldots<\alpha_{n}<\lambda, \alpha^{*} \leq \alpha_{m}$ then the set of $i<\delta$ such that:

(i') $(\exists l<m)\left(\lambda_{\eta_{\alpha_{l}} \uparrow i} \geq \lambda_{\eta_{\alpha_{m}} \uparrow i}\right)$ or

(ii') $F_{m}\left(\eta_{\alpha_{0}}\left\lceil(i+1), \ldots, \eta_{\alpha_{m-1}} \uparrow(i+1), \eta_{\alpha_{m}}\left\lceil i, \ldots, \eta_{\alpha_{n}}\lceil i) \in I_{\eta_{\alpha_{m}} \uparrow i}\right.\right.\right.$ (and well defined) but

$\eta_{\alpha_{m}} \uparrow(i+1) \in F_{m}\left(\eta_{\alpha_{0}} \uparrow(i+1), \ldots, \eta_{\alpha_{m-1}} \uparrow(i+1), \eta_{\alpha_{m}}\left\lceil i, \ldots, \eta_{\alpha_{n}}\lceil i)\right.\right.$,

is in the ideal $J$.

(8) The sequence $\bar{\eta}$ is big $J$-cofinal in $(T, \bar{\lambda}, \bar{I})$ if

(a) $\eta_{\alpha} \neq \eta_{\beta}$ for distinct $\alpha, \beta<\lambda$,

(b) for every $n<\omega$ and functions $F_{0}, \ldots, F_{n}$ there is $\alpha^{*}$ such that if $\alpha_{0}<\ldots<\alpha_{n}$ and $\alpha^{*} \leq \alpha_{m}, m \leq n$ then the set

$$
\left\{i<\delta: \eta_{\alpha_{m}}(i) \in F_{m}\left(\nu_{l}\right)_{l \leq n} \in I_{\eta_{\alpha_{m}} \backslash i}\right\}
$$

is in the ideal $J$, where

$$
\nu_{l}= \begin{cases}\eta_{\alpha_{l}}\lceil(i+1) & \text { if } \lambda_{\eta_{\alpha_{l}} \uparrow i}<\lambda_{\eta_{\alpha_{m}} \uparrow i} \text { or } \\ & \lambda_{\eta_{\alpha_{l}} \uparrow i}=\lambda_{\eta_{\alpha_{m}} \uparrow i} \text { and } \eta_{\alpha_{l}}(i)<\eta_{\alpha_{m}}(i), \\ \eta_{\alpha_{l}}\lceil i & \text { if not. }\end{cases}
$$


(9) In almost the same way we define "strongly* $J$-cofinal", "stronger* $J$-cofinal" and "strongest* big $J$-cofinal", replacing the requirement that $\alpha^{*} \leq \alpha_{m}$ in 5(b), 6(b), 7(b) above (respectively) by $\alpha^{*} \leq \alpha_{0}$.

REMARK 2.3. (a) Note that "strongest $J$-cofinal" implies "stronger $J$ cofinal" and this implies "strongly $J$-cofinal". "Stronger $J$-cofinal" implies "J-cofinal". Also "bigger" $\Rightarrow$ "big" $\Rightarrow$ "cofinal", "big" $\Rightarrow$ "strongly".

(b) The different notions of "strong $J$-cofinality" (the conditions (i) and $\left(\mathrm{i}^{\prime}\right)$ ) are to allow us to carry some diagonalization arguments.

(c) The difference between "strongly $J$-cofinal" and "strongly* $J$-cofinal" etc. is, in our context, immaterial. We may in all places in this paper replace the relevant notion with its version with "*" and no harm will be done.

REMARK 2.4. (1) Recall pcf: An important case is when $\left\langle\lambda_{i}: i<\delta\right\rangle$ is an increasing sequence of regular cardinals, $\lambda_{i}>\prod_{j<i} \lambda_{j}, \lambda_{\eta}=\lambda_{\lg (\eta)}$, $I_{\eta}=J_{\lambda_{\eta}}^{\mathrm{bd}}$ and $\lambda=\operatorname{tcf}\left(\prod_{i<\delta} \lambda_{i} / J\right)$.

(2) Moreover we are interested in more complicated $I_{\eta}$ 's (as in $[23, \S 5]$ ), connected to our problem, so "the existence of the true cofinality" is less clear. But the assumption $2^{\mu}=\mu^{+}$will rescue us.

(3) There are natural stronger demands of cofinality since here we are not interested just in $x_{\alpha}$ 's but also in Boolean combinations. Thus naturally we are interested in behaviours of large sets of $n$-tuples (see 5.1).

Proposition 2.5. Suppose that $(T, \bar{\lambda}, \bar{I}) \in \mathcal{K}_{\mu, \delta}^{\mathrm{id}}, \bar{\eta}=\left\langle\eta_{\alpha}: \alpha<\lambda\right\rangle \subseteq$ $\lim _{\delta}(T)$ and $J$ is an ideal on $\delta, J \supseteq J_{\delta}^{\mathrm{bd}}$.

(1) Assume that

(๑) if $\alpha<\beta<\lambda$ then $\left(\forall^{J} i<\delta\right)\left(\lambda_{\eta_{\alpha} \backslash i}<\lambda_{\eta_{\beta} \backslash i}\right)$.

Then the following are equivalent:

- " $\bar{\eta}$ is strongly $J$-cofinal for $(T, \bar{\lambda}, \bar{I})$ ",

- " $\bar{\eta}$ is stronger $J$-cofinal for $(T, \bar{\lambda}, \bar{I})$ ",

- " $\bar{\eta}$ is strongest $J$-cofinal for $(T, \bar{\lambda}, \bar{I})$ ",

- " " $\bar{\eta}$ is big J-cofinal for $(T, \bar{\lambda}, \bar{I})$ ".

(2) If $I_{\nu} \supseteq J_{\lambda_{\nu}}^{\mathrm{bd}}$ and $\lambda_{\nu}=\lambda_{\lg (\nu)}$ for each $\nu \in T$ and the sequence $\bar{\eta}$ is stronger $J$-cofinal for $(T, \bar{\lambda}, \bar{I})$ then for some $\alpha^{*}<\lambda$ the sequence $\left\langle\eta_{\alpha}: \alpha^{*} \leq\right.$ $\alpha<\lambda\rangle$ is $<_{J}$-increasing.

(3) If $\eta \in T_{i} \Rightarrow \lambda_{\eta}=\lambda_{i}$ and $\bar{\eta}$ is $<_{J}$-increasing in $\prod_{i<\delta} \lambda_{i}$ then "big" is equivalent to "stronger".

Proposition 2.6. Suppose that:

(1) $\left\langle\lambda_{i}: i<\delta\right\rangle$ is an increasing sequence of regular cardinals, where $\delta<\lambda_{0}$ is a limit ordinal,

(2) $T=\bigcup_{i<\delta} \prod_{j<i} \lambda_{j}, I_{\eta}=I_{\lg (\eta)}=J_{\lambda_{\lg (\eta)}}^{\mathrm{bd}}$ and $\lambda_{\eta}=\lambda_{\lg (\eta)}$, 
(3) $J$ is an ideal on $\delta, \lambda=\operatorname{tcf}\left(\prod_{i<\delta} \lambda_{i} / J\right)$ and it is exemplified by a sequence $\bar{\eta}=\left\langle\eta_{\alpha}: \alpha<\lambda\right\rangle \subseteq \prod_{i<\delta} \lambda_{i}$,

(4) $\left|\left\{\eta_{\alpha} \mid i: \alpha<\lambda\right\}\right|<\lambda_{i}$ for each $i<\delta$ (so, e.g., $\lambda_{i}>\prod_{j<i} \lambda_{j}$ suffices).

Then the sequence $\bar{\eta}$ is $J$-cofinal in $(T, \bar{\lambda}, \bar{I})$.

P r o of. First note that our assumptions imply that each ideal $I_{\eta}=I_{\lg (\eta)}$ is $\left|\left\{\eta_{\alpha} \mid \lg (\eta): \alpha<\lambda\right\}\right|^{+}$-complete. Hence for each sequence $\bar{A}=\left\langle A_{\eta}\right.$ : $\eta \in T\rangle \in \prod_{\eta \in T} I_{\eta}$ and $i<\delta$ the set

$$
A_{i}:=\bigcup\left\{A_{\eta_{\alpha} \backslash i}: \alpha<\lambda\right\}
$$

is in the ideal $I_{i}$, i.e., it is bounded in $\lambda_{i}$ (for $i<\delta$ ). (We should remind here our convention that we do not distinguish $\lambda_{i}$ and $\operatorname{succ}_{T}(\eta)$ if $\lg (\eta)=i$, see 2.1.) Take $\eta^{*} \in \prod_{i<\delta} \lambda_{i}$ such that for each $i<\delta$ we have $A_{i} \subseteq \eta^{*}(i)$. As the sequence $\bar{\eta}$ realizes the true cofinality of $\prod_{i<\delta} \lambda_{i} / J$ we find $\alpha^{*}<\lambda$ such that

$$
\alpha^{*} \leq \alpha<\lambda \Rightarrow\left\{i<\delta: \eta_{\alpha}(i)<\eta^{*}(i)\right\} \in J,
$$

which allows us to finish the proof.

It follows from the above proposition that the notion of $J$-cofinal sequence is not empty. Of course, it is better to have "strongly (or even: stronger) $J$-cofinal" sequences $\bar{\eta}$. So it is nice to find that sometimes the weaker notion implies the stronger one.

Proposition 2.7. Assume that $\delta$ is a limit ordinal, $\mu$ is a cardinal, and $(T, \bar{\lambda}, \bar{I}) \in \mathcal{K}_{\mu, \delta}^{\mathrm{id}}$. Let $J$ be an ideal on $\delta$ such that $J \supseteq J_{\delta}^{\mathrm{bd}}$ (which is our standard hypothesis). Further suppose that

$(\circledast) \quad$ if $\eta \in T_{i}$ then the ideal $I_{\eta}$ is $\left(\left|T_{i}\right|+\sum\left\{\lambda_{\nu}: \nu \in T_{i} \& \lambda_{\nu}<\lambda_{\eta}\right\}\right)^{+}$. complete.

Then each $J$-cofinal sequence $\bar{\eta}$ for $(T, \bar{\lambda}, \bar{I})$ is strongly $J$-cofinal for $(T, \bar{\lambda}, \bar{I})$.

If , in addition, $\eta \neq \nu \in T_{i} \Rightarrow \lambda_{\eta} \neq \lambda_{\nu}$ then $\bar{\eta}$ is big J-cofinal for $(T, \bar{\lambda}, \bar{I})$. Also, if in addition

$\eta \in T_{i} \quad \Rightarrow \quad\left(\exists^{! 1} \nu \in T_{i}\right)\left(\lambda_{\nu}=\lambda_{\eta}\right) \vee\left[\left(\exists^{\leq \lambda_{\eta}} \nu \in T_{i}\right)\left(\lambda_{\nu}=\lambda_{\eta}\right) \& I_{\eta}\right.$ normal $]$ then $\bar{\eta}$ is big J-cofinal.

Proof. Let $n<\omega$ and $F_{0}, \ldots, F_{n}$ be $(n+1)$-place functions. First we define a sequence $\bar{A}=\left\langle A_{\eta}: \eta \in T\right\rangle$. For $m \leq n$ and a sequence $\left\langle\eta_{m}, \ldots, \eta_{n}\right\rangle \subseteq T_{i}$ we put

$$
\begin{aligned}
A_{\left\langle\eta_{m}, \ldots, \eta_{n}\right\rangle}^{m}=\bigcup\left\{F_{m}\right. & \left(\nu_{0}, \ldots, \nu_{m-1}, \eta_{m}, \ldots, \eta_{n}\right): \nu_{0}, \ldots, \nu_{m-1} \in T_{i+1}, \\
& \left(\nu_{0}, \ldots, \nu_{m-1}, \eta_{m}, \ldots, \eta_{n}\right) \in \operatorname{dom}(F), \\
& \lambda_{\nu_{0} \uparrow i}<\lambda_{\eta}, \ldots, \lambda_{\nu_{m-1} \uparrow i}<\lambda_{\eta_{m}} \\
& \text { and } \left.F\left(\nu_{0}, \ldots, \nu_{m-1}, \eta_{m}, \ldots, \eta_{n}\right) \in I_{\eta_{m}}\right\}
\end{aligned}
$$


and next for $\eta \in T_{i}$ let

$$
A_{\eta}=\bigcup\left\{A_{\left\langle\eta, \eta_{m+1}, \ldots, \eta_{n}\right\rangle}^{m}: m \leq n \& \eta_{m+1}, \ldots, \eta_{n} \in T_{i}\right\} .
$$

Note that the assumption $(\circledast)$ was set up so that $A_{\left\langle\eta_{m}, \ldots, \eta_{n}\right\rangle}^{m} \in I_{\eta_{m}}$ and the sets $A_{\eta}$ are in $I_{\eta}$ (for $\eta \in T$ ).

By the $J$-cofinality of $\bar{\eta}$, for some $\alpha^{*}<\lambda$ we have

$$
\alpha^{*} \leq \alpha<\lambda \Rightarrow\left(\forall^{J} i<\delta\right)\left(\eta_{\alpha}\left\lceil(i+1) \notin A_{\eta_{\alpha}\lceil i}\right) .\right.
$$

We are going to prove that this $\alpha^{*}$ is as required in the definition of strongly $J$-cofinal sequences. So suppose that $m \leq n, \alpha_{0}<\ldots<\alpha_{n}<\lambda$ and $\alpha^{*} \leq \alpha_{m}$. By the choice of $\alpha^{*}$ the set $A:=\left\{i<\delta: \eta_{\alpha_{m}} \uparrow(i+1) \in A_{\eta_{\alpha_{m}} \uparrow i}\right\}$ is in the ideal $J$. But if $i<\delta$ is such that

- $(\forall l<m)\left(\lambda_{\eta_{\alpha_{l}} \uparrow i}<\lambda_{\eta_{\alpha_{m}} \uparrow i}\right)$,

- $F\left(\eta_{\alpha_{0}} \uparrow(i+1), \ldots, \eta_{\alpha_{m-1}} \uparrow(i+1), \eta_{\alpha_{m}}\left\lceil i, \ldots, \eta_{\alpha_{n}}\lceil i) \in I_{\eta_{\alpha_{m}} \uparrow i}\right.\right.$, but

- $\eta_{\alpha_{m}} \uparrow(i+1) \in F\left(\eta_{\alpha_{0}} \uparrow(i+1), \ldots, \eta_{\alpha_{m-1}} \uparrow(i+1), \eta_{\alpha_{m}} \uparrow i, \ldots, \eta_{\alpha_{n}} \uparrow i\right)$

then clearly $\eta_{\alpha_{m}} \uparrow(i+1) \in A_{\left\langle\eta_{\alpha_{m}}\left\lceil i, \ldots, \eta_{\alpha_{n}}\lceil i\rangle\right.\right.}$ and so $i \in A$.

The "big" version should be clear too.

Proposition 2.8. Assume that $\mu$ is a strong limit uncountable cardinal and $\left\langle\mu_{i}: i<\delta\right\rangle$ is an increasing sequence of cardinals with limit $\mu$. Further suppose that $(T, \bar{\lambda}, \bar{I}) \in \mathcal{K}_{\mu, \delta}^{\mathrm{id}},\left|T_{i}\right| \leq \mu_{i}($ for $i<\delta), \lambda_{\eta}<\mu$ and each $I_{\eta}$ is $\mu_{\lg (\eta)}^{+}$-complete and contains all singletons $($for $\eta \in T)$. Finally assume $2^{\mu}=\mu^{+}$and let $J$ be an ideal on $\delta, J \supseteq J_{\delta}^{\mathrm{bd}}$. Then there exists a stronger $J$-cofinal sequence $\bar{\eta}$ for $(T, \bar{\lambda}, \bar{I})$ of length $\mu^{+}\left(\right.$even for $\left.J=J_{\delta}^{\mathrm{bd}}\right)$. We can get "big" if

$$
\varrho \neq \eta \in T_{i} \& \lambda_{\varrho}=\lambda_{\eta} \quad \Rightarrow \quad\left(\exists^{\leq \lambda_{\eta}} \nu \in T_{i}\right)\left(\lambda_{\nu}=\lambda_{\eta}\right) \& I_{\eta} \text { normal. }
$$

Proof. This is a straight diagonal argument. Put

$$
\begin{aligned}
Y:=\left\{\left\langle F_{0}, \ldots, F_{n}\right\rangle:\right. & n<\omega \text { and each } F_{l} \text { is a function with } \\
& \left.\operatorname{dom}(F) \subseteq T^{n+1}, \operatorname{rng}(F) \subseteq \bigcup_{\eta \in T} I_{\eta}\right\} .
\end{aligned}
$$

Since $|Y|=\mu^{\mu}=\mu^{+}$(remember that $\mu$ is strong limit and $\lambda_{\eta}<\mu$ for $\eta \in T)$ we may choose an enumeration $Y=\left\{\left\langle F_{0}^{\xi}, \ldots, F_{n_{\xi}}^{\xi}\right\rangle: \xi<\mu^{+}\right\}$. For each $\zeta<\mu^{+}$choose an increasing sequence $\left\langle\mathcal{A}_{i}^{\zeta}: i<\delta\right\rangle$ such that $\left|\mathcal{A}_{i}^{\zeta}\right| \leq \mu_{i}$ and $\zeta=\bigcup_{i<\delta} \mathcal{A}_{i}^{\zeta}$. Now we choose by induction on $\zeta<\mu^{+}$branches $\eta_{\zeta}$ such that for each $\zeta$ the restriction $\eta_{\zeta}\lceil i$ is defined by induction on $i$ as follows.

If $i=0$ or $i$ is limit then there is nothing to do.

Suppose now that we have defined $\eta_{\zeta} \uparrow i$ and $\eta_{\xi}$ for $\xi<\zeta$. We find $\eta_{\zeta}(i)$ such that: 
$(\alpha) \eta_{\zeta}(i) \in \lambda_{\eta_{\zeta} \backslash i}$

$(\beta)$ if $\varepsilon \in \mathcal{A}_{i}^{\zeta}, m \leq n_{\varepsilon}, \alpha_{0}, \ldots, \alpha_{m-1} \in \mathcal{A}_{i}^{\zeta}$ (hence $\alpha_{l}<\zeta$ so $\eta_{\alpha_{l}}$ are already defined), $\nu_{m+1}, \ldots, \nu_{n} \in T_{i}$ and

$$
F_{m}^{\varepsilon}\left(\eta_{\alpha_{0}} \uparrow(i+1), \ldots, \eta_{\alpha_{m-1}} \uparrow(i+1), \eta_{\zeta}\left\lceil i, \nu_{m+1}, \ldots, \nu_{n}\right) \in I_{\eta_{\zeta} \uparrow i}\right.
$$

and well defined, then

$$
\eta_{\zeta} \uparrow(i+1) \notin F_{m}^{\varepsilon}\left(\eta_{\alpha_{0}} \uparrow(i+1), \ldots, \eta_{\alpha_{m-1}} \uparrow(i+1), \eta_{\zeta}\left\lceil i, \nu_{m+1}, \ldots, \nu_{n}\right),\right.
$$

$(\gamma) \eta_{\zeta} \uparrow(i+1) \notin\left\{\eta_{\varepsilon} \uparrow(i+1): \varepsilon \in \mathcal{A}_{i}^{\zeta}\right\}$.

Why is it possible? Note that there are $\leq \aleph_{0}+\left|\mathcal{A}_{i}^{\zeta}\right|+\left|\mathcal{A}_{i}^{\zeta}\right|<\aleph_{0}+\left|T_{i}\right| \leq$ $\mu_{i}$ negative demands and each of them says that $\eta_{\zeta} \uparrow(i+1)$ is in no set from $I_{\eta_{\zeta} \backslash i}$ (remember that we have assumed that the ideals $I_{\eta_{\zeta} \uparrow i}$ contain singletons). Consequently, using the completeness of the ideal we may satisfy the requirements $(\alpha)-(\gamma)$ above.

Now of course $\eta_{\zeta} \in \lim _{\delta}(T)$. Moreover if $\varepsilon<\zeta<\mu^{+}$then $(\exists i<\delta)(\varepsilon \in$ $\left.\mathcal{A}_{i}^{\zeta}\right)$, which implies $(\exists i<\delta)\left(\eta_{\varepsilon}\left\lceil(i+1) \neq \eta_{\zeta}\lceil(i+1))\right.\right.$. Consequently,

$$
\varepsilon<\zeta<\mu^{+} \Rightarrow \eta_{\varepsilon} \neq \eta_{\zeta} .
$$

Checking the demand (b) of "stronger $J$-cofinal" is straightforward: for functions $F_{0}, \ldots, F_{n}$ (and $n \in \omega$ ) take $\varepsilon$ such that

$$
\left\langle F_{0}, \ldots, F_{n}\right\rangle=\left\langle F_{0}^{\varepsilon}, \ldots, F_{n_{\varepsilon}}^{\varepsilon}\right\rangle
$$

and put $\alpha^{*}=\varepsilon+1$. Suppose now that $m \leq n, \alpha_{0}<\ldots<\alpha_{n}<\lambda$, $\alpha^{*} \leq \alpha_{m}$. Let $i^{*}<\delta$ be such that for $i>i^{*}$ we have

$$
\varepsilon, \alpha_{0}, \ldots, \alpha_{m-1} \in \mathcal{A}_{i}^{\alpha_{m}} \text {. }
$$

Then by the choice of $\eta_{\alpha_{m}}\left\lceil(i+1)\right.$ we see that for each $i>i^{*}$, if

$$
F_{m}^{\varepsilon}\left(\eta_{\alpha_{0}} \uparrow(i+1), \ldots, \eta_{\alpha_{m-1}} \uparrow(i+1), \eta_{\zeta}\left\lceil i, \eta_{\alpha_{m+1}}\left\lceil i, \ldots, \eta_{\alpha_{n}}\lceil i) \in I_{\eta_{\alpha_{m}} \uparrow},\right.\right.\right.
$$

then

$$
\eta_{\alpha_{m}}\left\lceili \notin F _ { m } ^ { \varepsilon } \left(\eta_{\alpha_{0}} \uparrow(i+1), \ldots, \eta_{\alpha_{m-1}} \uparrow(i+1), \eta_{\zeta}\left\lceil i, \eta_{\alpha_{m+1}}\left\lceil i, \ldots, \eta_{\alpha_{n}}\lceil i) .\right.\right.\right.\right.
$$

REMARK 2.9. The proof above can be carried out for functions $F$ which depend on $\left(\eta_{\alpha_{0}}, \ldots, \eta_{\alpha_{m-1}}, \eta_{\alpha_{m}} \backslash i, \ldots, \eta_{\alpha_{n}} \backslash i\right)$. This will be natural later.

Let us note that if the ideals $I_{\eta}$ are sufficiently complete then $J$-cofinal sequences cannot be too short.

Proposition 2.10. Suppose that $(T, \bar{\lambda}, \bar{I}) \in \mathcal{K}_{\mu, \delta}^{\mathrm{id}}$ is such that for each $\eta \in T_{i}, i<\delta$, the ideal $I_{\eta}$ is $\left(\kappa_{i}\right)^{+}$-complete $\left(\left[\lambda_{\eta}\right]^{\kappa_{i}} \subseteq I_{\eta}\right.$ is enough $)$. Let $J \supseteq J_{\delta}^{\text {bd }}$ be an ideal on $\delta$ and let $\bar{\eta}=\left\langle\eta_{\alpha}: \alpha<\delta^{*}\right\rangle$ be a $J$-cofinal sequence for $(T, \bar{\lambda}, \bar{I})$. Then

$$
\delta^{*}>\limsup _{J} \kappa_{i} \text { and consequently } \quad \operatorname{cf}\left(\delta^{*}\right)>\limsup _{J} \kappa_{i} .
$$


Proof. Fix an enumeration $\delta^{*}=\left\{\alpha_{\varepsilon}: \varepsilon<\left|\delta^{*}\right|\right\}$ and for $\alpha<\delta^{*}$ let $\zeta(\alpha)$ be the unique $\zeta$ such that $\alpha=\alpha_{\zeta}$. For $\eta \in T_{i}, i<\delta$, put

$$
A_{\eta}:=\left\{\nu \in \operatorname{succ}_{T}(\eta):\left(\exists \varepsilon \leq \kappa_{i}\right)\left(\nu \triangleleft \eta_{\varepsilon}\right)\right\} .
$$

Clearly $\left|A_{\bar{\eta}}\right| \leq \kappa_{i}$ and hence $A_{\eta} \in I_{\eta}$. Apply the $J$-cofinality of $\bar{\eta}$ to the sequence $\bar{A}=\left\langle A_{\eta}: \eta \in T\right\rangle$. Thus there is $\alpha^{*}<\delta^{*}$ such that for each $\alpha \in\left[\alpha^{*}, \delta^{*}\right)$ we have

$$
\left(\forall^{J} i<\delta\right)\left(\eta_{\alpha}\left\lceil(i+1) \notin A_{\eta_{\alpha} \uparrow i}\right)\right.
$$

and hence $\left(\forall^{J} i<\delta\right)\left(\zeta(\alpha)>\kappa_{i}\right)$ and consequently

$$
\zeta(\alpha) \geq \lim \sup _{J} \kappa_{i} \text {. }
$$

Hence we conclude that $\left|\delta^{*}\right|>\lim \sup _{J} \kappa_{i}$.

For the "consequently" part of the proposition note that if $\left\langle\eta_{\alpha}: \alpha<\delta^{*}\right\rangle$ is $J$-cofinal (in $(T, \bar{\lambda}, \bar{I})$ ) and $A \subseteq \delta^{*}$ is cofinal in $\delta^{*}$ then $\left\langle\eta_{\alpha}: \alpha \in A\right\rangle$ is $J$-cofinal too.

REMARK 2.11. (1) So if we have a $J$-cofinal sequence of length $\delta^{*}$ then we also have one of length $\operatorname{cf}\left(\delta^{*}\right)$. Thus assuming regularity of the length is natural.

(2) Moreover the assumption that the length of the sequence is above $|\delta|+$ $|T|$ is very natural and in most cases it will follow from the $J$-cofinality (and completeness assumptions). However we will try to state this condition in the assumptions whenever it is used in the proof (even if it can be concluded from the other assumptions).

\section{Getting $(\kappa, \operatorname{not} \lambda)$-Knaster algebras}

Proposition 3.1. Let $\lambda, \sigma$ be cardinals such that $(\forall \alpha<\sigma)\left(2^{|\alpha|}<\lambda\right)$ and $\sigma$ is regular. Then there are a Boolean algebra $\mathbb{B}$, a sequence $\left\langle y_{\alpha}: \alpha<\lambda\right\rangle \subseteq$ $\mathbb{B}^{+}$and an ideal $I$ on $\lambda$ such that:

(a) if $X \subseteq \lambda, X \notin I$ then $(\exists \alpha, \beta \in X)\left(\mathbb{B} \models y_{\alpha} \cap y_{\beta}=\mathbf{0}\right)$,

(b) the ideal $I$ is $\sigma$-complete,

(c) the algebra $\mathbb{B}$ satisfies the $\mu$-Knaster condition for any regular uncountable $\mu$ (actually, $\mathbb{B}$ is free).

Proof. Let $\mathbb{B}$ be the Boolean algebra freely generated by $\left\{z_{\alpha}: \alpha<\lambda\right\}$ (so the demand (c) is satisfied). Let $A=\{(\alpha, \beta): \alpha<\beta<\lambda\}$ and $y_{(\alpha, \beta)}=z_{\alpha}-z_{\beta}(\neq \mathbf{0})$ (for $\left.(\alpha, \beta) \in A\right)$. The ideal $I$ of subsets of $A$ is defined by:

- a set $X \subseteq A$ is in $I$ if there are $\zeta<\sigma, X_{\varepsilon} \subseteq A$ (for $\varepsilon<\zeta$ ) such that $X \subseteq \bigcup_{\varepsilon<\zeta} X_{\varepsilon}$ and for every $\varepsilon<\zeta$ no two $y_{\left(\alpha_{1}, \beta_{1}\right)}, y_{\left(\alpha_{2}, \beta_{2}\right)} \in X_{\varepsilon}$ are disjoint in $\mathbb{B}$. 
First note that

Claim 3.1.1. $A \notin I$.

Proof. If not then we have witnesses $\zeta<\sigma$ and $X_{\varepsilon}$ (for $\varepsilon<\zeta$ ) for it. So $A=\bigcup_{\varepsilon<\zeta} X_{\varepsilon}$ and hence for $(\alpha, \beta) \in A$ we have $\varepsilon(\alpha, \beta)$ such that $y_{(\alpha, \beta)} \in X_{\varepsilon(\alpha, \beta)}$. So $\varepsilon(\cdot, \cdot)$ is actually a function from $[\lambda]^{2}$ to $\zeta<\sigma$. By the Erdös-Rado theorem we find $\alpha<\beta<\gamma<\lambda$ such that $\varepsilon(\alpha, \beta)=\varepsilon(\beta, \gamma)$. But

$$
y_{(\alpha, \beta)} \cap y_{(\beta, \gamma)}=\left(z_{\alpha}-z_{\beta}\right) \cap\left(z_{\beta}-z_{\gamma}\right)=\mathbf{0},
$$

so $(\alpha, \beta),(\beta, \gamma)$ cannot be in the same $X_{\varepsilon}$-a contradiction.

To finish the proof note that $I$ is $\sigma$-complete (as $\sigma$ is regular), and if $X \notin I$ then, by the definition of $I$, there are two disjoint elements in $\left\{y_{(\alpha, \beta)}:(\alpha, \beta) \in X\right\}$. Finally $|A|=\lambda$.

Definition 3.2. (a) A pair $(\mathbb{B}, \bar{y})$ is called a $\lambda$-marked Boolean algebra if $\mathbb{B}$ is a Boolean algebra and $\bar{y}=\left\langle y_{\alpha}: \alpha\langle\lambda\rangle\right.$ is a sequence of non-zero elements of $\mathbb{B}$.

(b) A triple $(\mathbb{B}, \bar{y}, I)$ is called a $(\lambda, \chi)$-well marked Boolean algebra if $(\mathbb{B}, \bar{y})$ is a $\lambda$-marked Boolean algebra, $\chi$ is a regular cardinal and $I$ is a (proper) $\chi$-complete ideal on $\lambda$ such that

$$
\left\{A \subseteq \lambda:(\forall \alpha, \beta \in A)\left(\mathbb{B} \models y_{\alpha} \cap y_{\beta} \neq \mathbf{0}\right)\right\} \subseteq I .
$$

By a $\lambda$-well marked Boolean algebra we will mean a $\left(\lambda, \aleph_{0}\right)$-well marked one. As in the above situation $\lambda$ can be read off from $\bar{y}($ as $\lambda=\lg (\bar{y})$ ) we may omit it and then we may speak just about well marked Boolean algebras.

REMARK 3.3. Thus Proposition 3.1 says that if $\lambda, \sigma$ are regular cardinals and

$$
(\forall \alpha<\sigma)\left(2^{|\alpha|}<\lambda\right)
$$

then there exists a $(\lambda, \sigma)$-well marked Boolean algebra $(\mathbb{B}, \bar{y}, I)$ such that $\mathbb{B}$ has the $\kappa$-Knaster property for every $\kappa$.

Definition 3.4. (1) For cardinals $\mu$ and $\lambda$ and a limit ordinal $\delta$, a $(\delta, \mu, \lambda)$-constructor is a system

$$
\mathcal{C}=\left(T, \bar{\lambda}, \bar{\eta},\left\langle\left(\mathbb{B}_{\eta}, \bar{y}_{\eta}\right): \eta \in T\right\rangle\right)
$$

such that:

(a) $(T, \bar{\lambda}) \in \mathcal{K}_{\mu, \delta}$,

(b) $\bar{\eta}=\left\langle\eta_{i}: i \in \lambda\right\rangle$ where $\eta_{i} \in \lim _{\delta}(T)$ (for $i<\lambda$ ) are distinct $\delta$-branches through $T$,

(c) for each $\eta \in T,\left(\mathbb{B}_{\eta}, \bar{y}_{\eta}\right)$ is a $\lambda_{\eta}$-marked Boolean algebra, i.e., $\bar{y}_{\eta}=\left\langle y_{\eta}-\langle\alpha\rangle: \alpha<\lambda_{\eta}\right\rangle \subseteq \mathbb{B}_{\eta}^{+}$(usually this will be an enumeration of $\left.\mathbb{B}_{\eta}^{+}\right)$. 
(2) Let $\mathcal{C}$ be a constructor (as above). We define Boolean algebras $\mathbb{B}_{2}=$ $\mathbb{B}^{\text {red }}=\mathbb{B}^{\text {red }}(\mathcal{C})$ and $\mathbb{B}_{1}=\mathbb{B}^{\text {green }}=\mathbb{B}^{\text {green }}(\mathcal{C})$ as follows.

$\mathbb{B}^{\text {red }}$ is the Boolean algebra freely generated by $\left\{x_{i}: i<\lambda\right\}$ except that if

$i_{0}, \ldots, i_{n-1}<\lambda, \quad \nu=\eta_{i_{0}}\left\lceil\zeta=\eta_{i_{1}}\left\lceil\zeta=\ldots=\eta_{i_{n-1}} \mid \zeta, \quad \mathbb{B}_{\nu} \models \bigcap_{l<n} y_{\eta_{i_{l}}} \uparrow(\zeta+1)=\mathbf{0}\right.\right.$

then $\bigcap_{l<n} x_{i_{l}}=\mathbf{0}$. [Note: we may demand that the sequence $\left\langle\eta_{i_{l}}(\zeta): l<n\right\rangle$ is strictly increasing, this will cause no difference.]

$\mathbb{B}^{\text {green }}$ is the Boolean algebra freely generated by $\left\{x_{i}: i<\lambda\right\}$ except that if

$$
\nu=\eta_{i}\left\lceil\zeta=\eta_{j} \uparrow \zeta, \quad \eta_{i}(\zeta) \neq \eta_{j}(\zeta), \quad \mathbb{B}_{\nu} \models y_{\eta_{i} \uparrow(\zeta+1)} \cap y_{\eta_{j}} \uparrow(\zeta+1) \neq \mathbf{0}\right.
$$

then $x_{i} \cap x_{j}=\mathbf{0}$.

REMARK 3.5. (1) The equations for the green case can look strange but they have to be dual to the ones of the red case.

(2) "Freely generated except ..." means that a Boolean combination is non-zero except when some (finitely many) conditions imply it. For this it is enough to look at elements of the form

$$
x_{i_{0}}^{\mathfrak{t}_{0}} \cap \ldots \cap x_{i_{n-1}}^{\mathfrak{t}_{n-1}}
$$

where $\mathfrak{t}_{l} \in\{0,1\}$.

(3) Working in the free product $\mathbb{B}^{\text {red }} * \mathbb{B}^{\text {green }}$ we will use the same notation for elements (e.g., generators) of $\mathbb{B}^{\text {red }}$ as for elements of $\mathbb{B}^{\text {green }}$. Thus $x_{i}$ may stand either for the corresponding generator in $\mathbb{B}^{\text {red }}$ or $\mathbb{B}^{\text {green }}$. We hope that this will not be confusing, as one can easily decide in which algebra the element is considered from the place of it (if $x \in \mathbb{B}^{\text {red }}, y \in \mathbb{B}^{\text {green }}$ then $(x, y)$

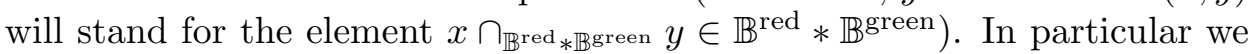
may write $\left(x_{i}, x_{i}\right)$ for an element which could be denoted by $x_{i}^{\text {red }} \cap x_{i}^{\text {green }}$.

REMARK 3.6. If the pair $\left(\mathbb{B}^{\text {red }}, \mathbb{B}^{\text {green }}\right)$ is a counterexample with the free product $\mathbb{B}^{\text {red }} * \mathbb{B}^{\text {green }}$ failing the $\lambda$-cc but each of the algebras satisfying that condition then each of the algebras fails the $\lambda$-Knaster condition. But $\mathbb{B}^{\text {red }}$ is supposed to have the $\kappa$-cc $(\kappa$ smaller than $\lambda)$. This is known to restrict $\lambda$.

Proposition 3.7. Assume that $\mathcal{C}=\left(T, \bar{\lambda}, \bar{\eta},\left\langle\left(\mathbb{B}_{\eta}, \bar{y}_{\eta}\right): \eta \in T\right\rangle\right)$ is a $(\delta, \mu, \lambda)$-constructor and $J \supseteq J_{\delta}^{\mathrm{bd}}$ is an ideal on $\delta$ such that:

(a) $\bar{\eta}=\left\langle\eta_{i}: i \in T\right\rangle$ is $J$-cofinal for $(T, \bar{\lambda}, \bar{I})$,

(b) if $X \in I_{\eta}^{+}$then

$$
(\exists \alpha, \beta \in X)\left(\mathbb{B}_{\eta}=y_{\eta}\langle\alpha\rangle \cap y_{\eta}\langle\beta\rangle=\mathbf{0}\right) .
$$

Then the sequence $\left\langle x_{\alpha}^{\mathrm{red}}: \alpha<\lambda\right\rangle$ exemplifies that $\mathbb{B}^{\mathrm{red}}(\mathcal{C})$ fails the $\lambda$-Knaster condition. 
Explanation. The above proposition is not just something in the direction of Problem 0.6. The tuple $\left(\mathbb{B}^{\text {red }}, \bar{x}, J_{\lambda}^{\text {bd }}\right)$ is like $\left(\mathbb{B}_{\eta}, \bar{y}_{\eta}, I_{\eta}\right)$, but $J_{\lambda}^{\text {bd }}$ is nicer than the ideals given by previous results. Using such objects makes building examples for Problem 0.6 much easier.

Pro of (of Proposition 3.7). It is enough to show that for each $Y \in[\lambda]^{\lambda}$ one can find $\varepsilon, \zeta \in Y$ such that

$$
\mathbb{B}_{\eta_{\varepsilon} \backslash i} \models y_{\eta_{\varepsilon} \uparrow(i+1)} \cap y_{\eta_{\zeta} \uparrow(i+1)}=\mathbf{0}
$$

where $i=\lg \left(\eta_{\varepsilon} \wedge \eta_{\zeta}\right)$. For this, for each $\nu \in T$ we put

$$
A_{\nu}:=\left\{\alpha<\lambda_{\nu}:(\exists \varepsilon \in Y)\left(\nu \curlyvee\langle\alpha\rangle \triangleleft \eta_{\varepsilon}\right)\right\} .
$$

Claim 3.7.1. There is $\nu \in T$ such that $A_{\nu} \notin I_{\nu}$.

Proof. First note that by the definition of $A_{\nu}$, for each $\varepsilon \in Y$ we have

$$
(\forall i<\delta)\left(\eta_{\varepsilon} \prec\langle i\rangle \in A_{\eta_{\varepsilon} \backslash i}\right) .
$$

Now, if we had $A_{\nu} \in I_{\nu}$ for all $\nu \in T$ then we could apply the assumption that $\bar{\eta}$ is $J$-cofinal for $(T, \bar{\lambda}, \bar{I})$ to the sequence $\left\langle A_{\nu}: \nu \in T\right\rangle$. Thus we would find $\alpha^{*}<\lambda$ such that

$$
\alpha^{*} \leq \alpha<\lambda \quad \Rightarrow \quad\left\{i<\delta: \eta_{\alpha}(i) \notin A_{\eta_{\alpha} \uparrow i}\right\} \in J,
$$

which contradicts our previous remark (remember $|Y|=\lambda$ ).

Due to the claim we find $\nu \in T$ such that $A_{\nu} \notin I_{\nu}$. By part (b) of our assumptions we find $\alpha, \beta \in A_{\nu}$ such that

$$
\mathbb{B}_{\nu}=y_{\nu}\langle\alpha\rangle \cap y_{\nu}-\langle\beta\rangle=\mathbf{0} .
$$

Choose $\varepsilon, \zeta \in Y$ such that $\nu \frown\langle\alpha\rangle \triangleleft \eta_{\varepsilon}, \nu \frown\langle\beta\rangle \triangleleft \eta_{\zeta}$ (see the definition of $\left.A_{\nu}\right)$. Then $\nu=\eta_{\varepsilon} \wedge \eta_{\zeta}$ and

$$
\mathbb{B}_{\nu} \models y_{\eta_{\varepsilon} \backslash(i+1)} \cap y_{\eta_{\zeta} \backslash(i+1)}=\mathbf{0}
$$

(where $i=\lg (\nu)$ ), finishing the proof of the proposition.

Lemma 3.8. Let $\mathcal{C}=\left(T, \bar{\lambda}, \bar{\eta},\left\langle\left(\mathbb{B}_{\eta}, \bar{y}_{\eta}\right): \eta \in T\right\rangle\right)$ be a $(\delta, \mu, \lambda)$-constructor such that

( $\star$ for $\eta \in T$, the Boolean algebras $\mathbb{B}_{\eta}$ satisfy the $\left(2^{|\delta|}\right)^{+}$-Knaster condition.

Then the Boolean algebra $\mathbb{B}^{\text {red }}(\mathcal{C})$ satisfies the $\left(2^{|\delta|}\right)^{+}-$Knaster condition. In fact we may replace $\left(2^{|\delta|}\right)^{+}$above by any regular cardinal $\theta$ such that

$$
(\forall \alpha<\theta)\left(|\alpha|^{|\delta|}<\theta\right) \text {. }
$$


To deduce that $\mathbb{B}^{\text {red }}(\mathcal{C})$ satisfies the $\left(2^{|\delta|}\right)^{+}-c c$ it is enough to assume, instead of $(\star)$,

$(\star \star)$ for $\eta \in T$, every free product of finitely many of the Boolean algebras $\mathbb{B}_{\eta}$ satisfies the $\left(2^{|\delta|}\right)^{+}$-cc.

REMARK. (1) Usually we will have $\delta=\operatorname{cf}(\mu)$.

(2) Later we will get more (e.g., $|\delta|^{+}$-Knaster if $(T, \bar{\eta})$ is hereditarily free, see $5.12,5.13)$.

Pro of (of Lemma 3.8). Let $\theta=\left(2^{|\delta|}\right)^{+}$and assume $(\star)$ (the other cases have the same proofs). Suppose that $z_{\varepsilon} \in \mathbb{B}^{\text {red }} \backslash\{\mathbf{0}\}$ (for $\varepsilon<\theta$ ). We start with a series of reductions which we describe fully here but later, in similar situations, we will state the result of the procedure only.

Standard cleaning. Each $z_{\varepsilon}$ is a Boolean combination of some generators $x_{i_{0}}, \ldots, x_{i_{n-1}}$. But, as we want to find a subsequence with non-zero intersections, we may replace $z_{\varepsilon}$ by any non-zero $z \leq z_{\varepsilon}$. Consequently, we may assume that each $z_{\varepsilon}$ is an intersection of some generators or their complements. Further, as $\operatorname{cf}(\theta)=\theta>\aleph_{0}$ we may assume that the number of generators needed for this representation does not depend on $\varepsilon$ and is equal to, say, $n^{*}$. Thus we have two functions

$$
i: \theta \times n^{*} \rightarrow \lambda \quad \text { and } \quad \mathfrak{t}: \theta \times n^{*} \rightarrow 2
$$

such that for each $\varepsilon<\theta$,

$$
z_{\varepsilon}=\bigcap_{l<n^{*}}\left(x_{i(\varepsilon, l)}\right)^{\mathfrak{t}(\varepsilon, l)}
$$

and there is no repetition in $\left\langle i(\varepsilon, l): l<n^{*}\right\rangle$. Moreover we may assume that $\mathfrak{t}(\varepsilon, l)$ does not depend on $\varepsilon$, i.e., $\mathfrak{t}(\varepsilon, l)=\mathfrak{t}(l)$. By the $\Delta$-system lemma for finite sets we may assume that $\left\langle\left\langle i(\varepsilon, l): l\left\langle n^{*}\right\rangle: \varepsilon\langle\theta\rangle\right.\right.$ is a $\Delta$-system of sequences, i.e.:

$(*)_{1} i\left(\varepsilon, l_{1}\right)=i\left(\varepsilon, l_{2}\right) \Rightarrow l_{1}=l_{2}$,

$(*)_{2}$ for some $w \subseteq n^{*}$ we have

$$
\begin{array}{rll}
\left(\exists \varepsilon_{1}<\varepsilon_{2}<\theta\right)\left(i\left(\varepsilon_{1}, l\right)=i\left(\varepsilon_{2}, l\right)\right) & \text { iff } \quad\left(\forall \varepsilon_{1}, \varepsilon_{2}<\theta\right)\left(i\left(\varepsilon_{1}, l\right)=i\left(\varepsilon_{2}, l\right)\right) \\
& \text { iff } l \in w .
\end{array}
$$

Now note that, by the definition of the algebra $\mathbb{B}^{\text {red }}$,

$(*)_{3} z_{\varepsilon_{1}} \cap z_{\varepsilon_{2}}=\mathbf{0}$ if and only if

$$
\bigcap\left\{x_{i\left(\varepsilon_{1}, l\right)}^{\mathfrak{t}(l)}: l<n^{*}, \mathfrak{t}(l)=0\right\} \cap \bigcap\left\{x_{i\left(\varepsilon_{2}, l\right)}^{\mathfrak{t}(l)}: l<n^{*}, \mathfrak{t}(l)=0\right\}=\mathbf{0} .
$$

Consequently, we may assume that

$$
\left(\forall l<n^{*}\right)(\forall \varepsilon<\theta)(\mathfrak{t}(l)=0) .
$$


Explanation of what we are going to do now. We want to replace the sequence $\left\langle z_{\varepsilon}: \varepsilon<\theta\right\rangle$ by a large subsequence such that the places of splitting between two branches used in two different $z_{\varepsilon}$ 's will be uniform. Then we will be able to translate our $\theta$-cc problem to one on the algebras $\mathbb{B}_{\eta}$.

Let

$$
A_{\varepsilon}:=\left\{\nu \in{ }^{\delta>} \mu:(\exists j<\varepsilon)\left(\exists l<n^{*}\right)\left(\nu \triangleleft \eta_{i(j, l)}\right)\right\}
$$

and let $B_{\varepsilon}$ be the closure of $A_{\varepsilon}$ :

$$
\begin{aligned}
B_{\varepsilon}:=\left\{\varrho \in \in^{\delta \geq} \mu:\right. & \varrho \in A_{\varepsilon} \text { or } \lg (\varrho) \text { is a limit ordinal and } \\
& (\forall \zeta<\lg (\varrho))\left(\varrho\left\lceil\zeta \in A_{\varepsilon}\right)\right\}
\end{aligned}
$$

Note that $\left|A_{\varepsilon}\right| \leq|\varepsilon| \cdot|\delta|$ and hence $\left|B_{\varepsilon}\right| \leq\left|A_{\varepsilon}\right| \leq|\delta|<\theta$. Next we define (for $\left.\varepsilon<\theta, l<n^{*}\right)$

$$
\zeta(\varepsilon, l):=\sup \left\{\zeta<\delta: \eta_{i(\varepsilon, l)}\left\lceil\zeta \in B_{\varepsilon}\right\} .\right.
$$

Thus $\zeta(\varepsilon, l) \leq \lg \left(\eta_{i(\varepsilon, l)}\right)=\delta$. Let $\mathcal{S}=\{\varepsilon<\theta: \operatorname{cf}(\varepsilon)>|\delta|\}$. For each $\varepsilon \in \mathcal{S}$ we necessarily have

$$
\eta_{i(\varepsilon, l)}\left\lceil\zeta(\varepsilon, l) \in B_{\varepsilon} \quad \text { and } \quad B_{\varepsilon}=\bigcup_{\xi<\varepsilon} B_{\xi}\right.
$$

(remember that $\operatorname{cf}(\varepsilon)>|\delta|$ and for limit $\varepsilon$ we have $A_{\varepsilon}=\bigcup_{\xi<\varepsilon} A_{\xi}$ ) and hence

$$
\eta_{i(\varepsilon, l)}\left\lceil\zeta(\varepsilon, l) \in B_{\xi(\varepsilon, l)} \quad \text { for some } \xi(\varepsilon, l)<\varepsilon\right. \text {. }
$$

Let $\xi(\varepsilon)=\max \left\{\xi(\varepsilon, l): l<n^{*}\right\}$. By the Fodor lemma we find $\xi^{*}<\theta$ such that the set

$$
\mathcal{S}_{1}:=\left\{\varepsilon \in \mathcal{S}: \xi(\varepsilon)=\xi^{*}\right\}
$$

is stationary. Thus $\eta_{i(\varepsilon, l)}\left\lceil\zeta(\varepsilon, l) \in B_{\xi^{*}}\right.$ for each $\varepsilon \in \mathcal{S}_{1}, l<n^{*}$. Since $\left|B_{\xi^{*}}\right|,|\delta|<\theta$ we find $\nu_{0}, \ldots, \nu_{n^{*}-1} \in B_{\xi^{*}}$ and $\alpha\left(l_{1}, l_{2}\right) \leq \delta\left(\right.$ for $\left.l_{1} \leq l_{2}<n^{*}\right)$ such that the set

$$
\begin{aligned}
\mathcal{S}_{2}:=\left\{\varepsilon \in \mathcal{S}_{1}:\right. & \left(\forall l<n^{*}\right)\left(\eta_{i(\varepsilon, l)} \mid \zeta(\varepsilon, l)=\nu_{l}\right) \\
& \left.\&\left(\forall l_{1} \leq l_{2}<n^{*}\right)\left(\lg \left(\eta_{i\left(\varepsilon, l_{1}\right)} \wedge \eta_{i\left(\varepsilon, l_{2}\right)}\right)=\alpha\left(l_{1}, l_{2}\right)\right)\right\}
\end{aligned}
$$

is stationary. Further, applying the $\Delta$-system lemma we find a set $\mathcal{S}_{3} \in\left[\mathcal{S}_{2}\right]^{\theta}$ such that

$$
\left\{\left\langle\eta_{i(\varepsilon, l)}\left(\lg \left(\nu_{l}\right)\right): l<n^{*}\right\rangle: \varepsilon \in \mathcal{S}_{3}\right\}
$$

forms a $\Delta$-system of sequences.

For $\varepsilon \in \mathcal{S}_{3}$ and $\nu \in T$ define

$$
b_{\nu}^{\varepsilon}:=\bigcap\left\{y_{\eta_{i(\varepsilon, l)} \Gamma(\lg (\nu)+1)}: l<n^{*}, \nu \triangleleft \eta_{i(\varepsilon, l)}\right\} \in \mathbb{B}_{\nu} .
$$

Claim 3.8.1. For each $\varepsilon \in \mathcal{S}_{3}, \nu \in T$ the element $b_{\nu}^{\varepsilon}$ (of the algebra $\mathbb{B}_{\nu}$ ) is non-zero. 
Proof. This follows from the definition of $\mathbb{B}^{\text {red }}$ and the fact that $z_{\varepsilon} \neq \mathbf{0}$, as

$$
b_{\nu}^{\varepsilon}=\mathbf{0} \Rightarrow \bigcap\left\{x_{\eta_{i(\varepsilon, l)}}: l<n^{*}, \nu \triangleleft \eta_{i(\varepsilon, l)}\right\}=\mathbf{0} \quad \Rightarrow \quad z_{\varepsilon}=\mathbf{0} .
$$

Since for each $l<n^{*}$ the algebra $\mathbb{B}_{\nu_{l}}$ has the $\theta$-Knaster property we find a set $\mathcal{S}_{4} \in\left[\mathcal{S}_{3}\right]^{\theta}$ such that for each $l<n^{*}$ and $\varepsilon_{1}, \varepsilon_{2} \in \mathcal{S}_{4}$ we have

$$
\varepsilon_{1} \neq \varepsilon_{2} \quad \Rightarrow \quad b_{\nu_{l}}^{\varepsilon_{1}} \cap b_{\nu_{l}}^{\varepsilon_{2}} \neq \mathbf{0} \text { in } \mathbb{B}_{\nu_{l}} \text {. }
$$

Now we may finish by proving the following claim.

Claim 3.8.2. For each $\varepsilon_{1}, \varepsilon_{2} \in \mathcal{S}_{4}$,

$$
\mathbb{B}^{\text {red }} \models z_{\varepsilon_{1}} \cap z_{\varepsilon_{2}} \neq \mathbf{0} \text {. }
$$

P r o of. Since $z_{\varepsilon_{1}} \cap z_{\varepsilon_{2}}$ is just the intersection of generators it is enough to show that (remember the definition of $\mathbb{B}^{\text {red }}$ ):

(ه) for each $\varepsilon_{1}, \varepsilon_{2} \in \mathcal{S}_{4}$ and for every $\nu \in T$,

$$
\mathbb{B}_{\nu}=\bigcap\left\{y_{\eta_{i} \uparrow(\lg (\nu)+1)}: i \in\left\{i\left(\varepsilon_{1}, l\right), i\left(\varepsilon_{2}, l\right): l<n^{*}\right\} \text { and } \nu \triangleleft \eta_{i}\right\} \neq \mathbf{0} \text {. }
$$

If $\nu=\nu_{l}, l<n^{*}$ then the intersection is $b_{\nu_{l}}^{\varepsilon_{1}} \cap b_{\nu_{l}}^{\varepsilon_{2}}$, which is not zero by the choice of $\mathcal{S}_{4}$. So suppose that $\nu \notin\left\{\nu_{l}: l<n^{*}\right\}$. Put

$u_{\nu}:=\left\{i: \nu \triangleleft \eta_{i}\right.$ and for some $l<n^{*}$ either $i=i\left(\varepsilon_{1}, l\right)$ or $\left.i=i\left(\varepsilon_{2}, l\right)\right\}$.

If

$$
\left\{\eta_{i}(\lg (\nu)): i \in u_{\nu}\right\} \subseteq\left\{\eta_{i\left(\varepsilon_{2}, l\right)}(\lg (\nu)): l<n^{*} \& \nu \triangleleft \eta_{i\left(\varepsilon_{2}, l\right)}\right\}
$$

then we are done as $b_{\nu}^{\varepsilon_{2}} \neq \mathbf{0}$. So there is $l_{1}<n^{*}$ such that $\nu \triangleleft \eta_{i\left(\varepsilon_{1}, l_{1}\right)}$ and

$$
\eta_{i\left(\varepsilon_{1}, l_{1}\right)} \uparrow(\lg (\nu)+1) \notin\left\{\eta_{i\left(\varepsilon_{2}, l\right)} \uparrow(\lg (\nu)+1): l<n^{*} \& \nu \triangleleft \eta_{i\left(\varepsilon_{2}, l\right)}\right\} .
$$

Similarly we may assume that there is $l_{2}<n^{*}$ such that $\nu \triangleleft \eta_{i\left(\varepsilon_{2}, l_{2}\right)}$ and

$$
\eta_{i\left(\varepsilon_{2}, l_{2}\right)} \uparrow(\lg (\nu)+1) \notin\left\{\eta_{i\left(\varepsilon_{1}, l\right)} \uparrow(\lg (\nu)+1): l<n^{*} \& \nu \triangleleft \eta_{i\left(\varepsilon_{1}, l\right)}\right\} .
$$

By symmetry we may assume that $\varepsilon_{1}<\varepsilon_{2}$. Then

$$
\nu=\eta_{i\left(\varepsilon_{2}, l_{2}\right)} \uparrow \lg (\nu) \in A_{\varepsilon_{1}+1} \subseteq B_{\varepsilon_{2}}
$$

and hence $\zeta\left(\varepsilon_{2}, l_{2}\right) \geq \lg (\nu)$. By the choice of $\mathcal{S}_{2}$ (remember $\left.\varepsilon_{1}, \varepsilon_{2} \in \mathcal{S}_{4} \subseteq \mathcal{S}_{2}\right)$, we get $\nu \unlhd \nu_{l_{2}}$. But we have assumed that $\nu \neq \nu_{l_{2}}$, so $\nu \triangleleft \nu_{l_{2}}$. Hence (once again due to $\varepsilon_{1}, \varepsilon_{2} \in \mathcal{S}_{2}$ )

$$
\eta_{i\left(\varepsilon_{2}, l_{2}\right)}\left\lceil(\lg (\nu)+1)=\eta_{i\left(\varepsilon_{1}, l_{2}\right)}\left\lceil(\lg (\nu)+1)=\nu_{l_{2}} \uparrow(\lg (\nu)+1),\right.\right.
$$

which contradicts the choice of $l_{2}$.

This completes the proof of Lemma 3.8.

REMARK 3.9. We can strengthen " $\theta$-Knaster" in the assumption and conclusion of 3.8 in various ways. For example we may have "intersection of any $n$ members of the final set is non-zero". 
Definition 3.10. Let $(\mathbb{B}, \bar{y})$ be a $\lambda$-marked Boolean algebra, $\kappa \leq \lambda$. We say that:

(1) $(\mathbb{B}, \bar{y})$ the $\kappa$-Knaster property if $\mathbb{B}$ satisfies the condition in the definition of the $\kappa$-Knaster property (see 0.4 ) with restriction to subsequences of $\bar{y}$.

(2) $(\mathbb{B}, \bar{y})$ is $(\kappa, \operatorname{not} \lambda)$-Knaster if

(a) the algebra $\mathbb{B}$ has the $\kappa$-Knaster property, but

(b) the sequence $\bar{y}$ witnesses that the $\lambda$-Knaster property fails for $\mathbb{B}$.

CONCLUSION 3.11. Assume that $\mu$ is a strong limit singular cardinal, $\lambda=2^{\mu}=\mu^{+}$and $\theta=\left(2^{\operatorname{cf}(\mu)}\right)^{+}$. Then there exists a $\lambda$-marked Boolean algebra $(\mathbb{B}, \bar{y})$ which is $(\theta, \operatorname{not} \lambda)$-Knaster.

Proof. Choose cardinals $\mu_{i}^{0}, \mu_{i}<\mu$ (for $\left.i<\operatorname{cf}(\mu)\right)$ such that:

$(\alpha) \operatorname{cf}(\mu)<\mu_{0}^{0}$,

(ß) $\prod_{j<i} \mu_{j}<\mu_{i}^{0}, \mu_{i}=\left(2^{\mu_{i}^{0}}\right)^{+}$,

$(\gamma)\left\langle\mu_{i}: i<\operatorname{cf}(\mu)\right\rangle$ and $\left\langle\mu_{i}^{0}: i<\operatorname{cf}(\mu)\right\rangle$ are increasing cofinal in $\mu$.

(Possible as $\mu$ is strong limit singular.) By Proposition 3.1 we find $\mu_{i}$-marked Boolean algebras $\left(\mathbb{B}_{i}, \bar{y}^{i}\right)$ and $\left(\mu_{i}^{0}\right)^{+}$-complete ideals $I_{i}$ on $\mu_{i}($ for $i<\delta$ ) such that:

(a) if $X \subseteq \mu_{i}, X \notin I_{i}$ then $(\exists \alpha, \beta \in X)\left(\mathbb{B}_{i}=y_{\alpha}^{i} \cap y_{\beta}^{i}=\mathbf{0}\right)$,

(b) the algebra $\mathbb{B}_{i}$ has the $\left(2^{\mathrm{cf}(\mu)}\right)^{+}-$Knaster property.

Let $T=\bigcup_{i<\operatorname{cf}(\mu)} \prod_{j<i} \mu_{j}$ and for $\nu \in T_{i}(i<\operatorname{cf}(\mu))$ let $I_{\nu}=I_{i}, \mathbb{B}_{\nu}=\mathbb{B}_{i}$, $\bar{y}_{\nu}=\bar{y}^{i}$ and $\lambda_{\nu}=\mu_{i}$. Now we may apply Proposition 2.8 to $\mu,\left\langle\mu_{i}^{0}: i<\right.$ $\operatorname{cf}(\mu)\rangle$ and $(T, \bar{\lambda}, \bar{I})$ to find a stronger $J_{\mathrm{cf}(\mu)}^{\mathrm{bd}}$-cofinal sequence $\bar{\eta}$ for $(T, \bar{\lambda}, \bar{I})$ of length $\lambda$. Consider the $(\operatorname{cf}(\mu), \mu, \lambda)$-constructor $\mathcal{C}=\left(T, \bar{\lambda}, \bar{\eta},\left\langle\left(\mathbb{B}_{\nu}, \bar{y}_{\nu}\right)\right.\right.$ : $\nu \in T\rangle)$. By (b) above we may apply Lemma 3.8 to deduce that the algebra $\mathbb{B}^{\text {red }}(\mathcal{C})$ satisfies the $\left(2^{\mathrm{cf}(\mu)}\right)^{+}$-Knaster condition. Finally we use Proposition 3.7 (and (a) above) to conclude that $\left(\mathbb{B}^{\text {red }}(\mathcal{C}),\left\langle x_{\alpha}^{\text {red }}: \alpha<\lambda\right\rangle\right.$ ) is $(\theta, \operatorname{not} \lambda)$-Knaster.

Proposition 3.12. Assume that $\kappa$ is a regular cardinal such that $(\forall \alpha<$ $\kappa)\left(|\alpha|^{|\delta|}<\kappa\right), \bar{\lambda}=\left\langle\lambda_{i}: i<\delta\right\rangle$ is an increasing sequence of regular cardinals such that $\kappa \leq \lambda_{0}, \prod_{j<i} \lambda_{j}<\lambda_{i}$ (or just max $\operatorname{pcf}\left\{\lambda_{j}: j<i\right\}<\lambda_{i}$ ) for $i<\delta$ and $\lambda \in \operatorname{pcf}\left\{\lambda_{i}: i<\delta\right\}$. Further suppose that for each $i<\delta$ there exists a $\lambda_{i}$-marked Boolean algebra which is $\left(\kappa, \operatorname{not} \lambda_{i}\right)$-Knaster. Then there exists a $\lambda$-marked Boolean algebra which is $(\kappa, \operatorname{not} \lambda)$-Knaster.

Proof. If $\lambda=\lambda_{i}$ for some $i<\delta$ then there is nothing to do. If $\lambda<\lambda_{i}$ for some $i<\delta$ then let $\alpha<\delta$ be the maximal limit ordinal such that $(\forall i<\alpha)\left(\lambda_{i}<\lambda\right)$ (it necessarily exists). Now we may replace $\left\langle\lambda_{i}: i<\delta\right\rangle$ by 
$\left\langle\lambda_{i}: i<\alpha\right\rangle$. Thus we may assume that $(\forall i<\delta)\left(\lambda_{i}<\lambda\right)$. Further we may assume that

$$
\lambda=\max \operatorname{pcf}\left\{\lambda_{i}: i<\delta\right\}
$$

(by $[22, \mathrm{I}, 1.8]$ ). Now, due to $[22$, II, 3.5, p. 65], we find a sequence $\bar{\eta} \subseteq$ $\prod_{i<\delta} \lambda_{i}$ and an ideal $J$ on $\delta$ such that:

(1) $J \supseteq J_{\delta}^{\mathrm{bd}}$ and $\lambda=\operatorname{tcf}\left(\prod_{i<\delta} \lambda_{i} / J\right)$ (naturally: $J=\left\{a \subseteq \delta: \max \operatorname{pcf}\left\{\lambda_{i}: i \in a\right\}<\lambda\right\}$ ),

(2) $\bar{\eta}=\left\langle\eta_{\varepsilon}: \varepsilon<\lambda\right\rangle$ is $<_{J}$-increasing cofinal in $\prod_{i<\delta} \lambda_{i} / J$,

(3) for each $i<\delta,\left|\left\{\eta_{\varepsilon} \mid i: \varepsilon<\lambda\right\}\right|<\lambda_{i}$.

Let $T=\bigcup_{i<\delta} \prod_{j<i} \lambda_{j}$ and for $\nu \in T_{i}(i<\delta)$ let $\lambda_{\nu}=\lambda_{i}, I_{\nu}=J_{\lambda_{i}}^{\mathrm{bd}}$.

It follows from the choice of $\bar{\eta}, J$ above and our assumptions that we may apply Proposition 2.6 and hence $\bar{\eta}$ is $J$-cofinal for $(T, \bar{\lambda}, \bar{I})$. For $\nu \in T$ let $\left(\mathbb{B}_{\nu}, \bar{y}_{\nu}\right)$ be a $\lambda_{\nu}$-marked $\left(\kappa, \operatorname{not} \lambda_{\nu}\right)$-Knaster Boolean algebra (exists by our assumptions). Now we may finish using 3.8 and 3.7 for $\mathcal{C}=\left(T, \bar{\lambda}, \bar{\eta},\left\langle\left(\mathbb{B}_{\eta}, \bar{y}_{\eta}\right)\right.\right.$ : $\eta \in T\rangle$ ), $\bar{I}$ and $J$ (note the assumption (b) of 3.7 is satisfied as $I_{\eta}=J_{\lambda_{\eta}}^{\mathrm{bd}}$; remember the choice of $\left.\left(\mathbb{B}_{\eta}, \bar{y}_{\eta}\right)\right)$.

REMARK 3.13. Note that from the cardinal arithmetic hypothesis $\operatorname{cf}(\mu)$ $=\chi, \chi^{<\chi}<\chi<\mu, \mu^{+}=\lambda<2^{\chi}$ alone we cannot hope to build a counterexample. This is because of $[15, \S 4]$, particularly Lemma 4.13 there. It was shown in that paper that if $\chi^{<\chi}<\chi_{1}=\chi_{1}^{<\chi_{1}}$ then there is a $\chi^{+}$-cc $\chi$-complete forcing notion $\mathbb{P}$ of size $\chi_{1}$ such that

$$
\Vdash_{\mathbb{P}} \text { "if }|\mathbb{B}|<\chi_{1}, \mathbb{B} \models \chi \text {-cc then } \mathbb{B}^{+} \text {is the union of } \chi \text { ultrafilters". }
$$

More on this in Section 8. So the centrality of $\lambda \in \operatorname{Reg} \cap\left(\mu, 2^{\mu}\right], \mu$ strong limit singular, is very natural.

\section{The main result}

Proposition 4.1. Suppose that $\mathcal{C}$ is a $(\delta, \mu, \lambda)$-constructor. Then the free product $\mathbb{B}^{\text {red }}(\mathcal{C}) * \mathbb{B}^{\text {green }}(\mathcal{C})$ fails the $\lambda$-cc $\left(\operatorname{soc}\left(\mathbb{B}^{\text {red }}(\mathcal{C}) * \mathbb{B}^{\text {green }}(\mathcal{C})\right) \geq \lambda\right)$.

P r o o f. Look at the elements $\left(x_{i}, x_{i}\right) \in \mathbb{B}^{\text {red }} * \mathbb{B}^{\text {green }}$ for $i<\lambda$. It follows directly from the definition of the algebras that for each $i<j<\lambda$,

$$
\text { either } \quad \mathbb{B}^{\text {red }} \models x_{i}^{\text {red }} \cap x_{j}^{\text {red }}=\mathbf{0} \quad \text { or } \quad \mathbb{B}^{\text {green }} \models x_{i}^{\text {green }} \cap x_{j}^{\text {green }}=\mathbf{0} .
$$

Consequently, the sequence $\left\langle\left(x_{i}, x_{i}\right): i<\lambda\right\rangle$ witnesses the assertion.

Proposition 4.2. Suppose that $n<\omega$ and for $l \leq n$ :

(1) $\chi_{l}, \lambda_{l}$ are regular cardinals, $\chi_{l}<\lambda_{l}<\chi_{l+1}$,

(2) $\left(\mathbb{B}_{l}, \bar{y}_{l}, I_{l}\right)$ is a $\left(\lambda_{l}, \chi_{l}\right)$-well marked Boolean algebra (see Definition 3.2), $\bar{y}_{l}=\left\langle y_{i}^{l}: i<\lambda_{l}\right\rangle$, 
(3) $\mathbb{B}$ is the Boolean algebra freely generated by $\left\{y_{\eta}: \eta \in \prod_{l \leq n} \lambda_{l}\right\}$ except that if

$\eta_{i_{0}}, \ldots, \eta_{i_{k-1}} \in \prod_{l \leq n} \lambda_{l}, \quad \eta_{i_{0}}\left\lceil l=\eta_{i_{1}} \uparrow l=\ldots=\eta_{i_{n-1}} \uparrow l, \quad \mathbb{B}_{l} \models \bigcap_{m<k} y_{\eta_{i_{m}}(l)}^{l}=\mathbf{0}\right.$

then $\bigcap_{m<k} y \eta_{i_{m}}=\mathbf{0}$. [Compare the definition of the algebras $\mathbb{B}^{\text {red }}(\mathcal{C})$.]

(4) $I=\left\{B \subseteq \prod_{l \leq n} \lambda_{l}: \neg\left(\exists^{I_{0}} \gamma_{0}\right) \ldots\left(\exists^{I_{n}} \gamma_{n}\right)\left(\left\langle\gamma_{0}, \ldots, \gamma_{n}\right\rangle \in B\right)\right\}$.

Then:

(a) if all the algebras $\mathbb{B}_{l}($ for $l \leq n)$ have the $\theta$-Knaster property and $\theta$ is a regular uncountable cardinal then $\mathbb{B}$ has the $\theta$-Knaster property,

(b) $I$ is a $\chi_{0}$-complete ideal on $\prod_{l \leq n} \lambda_{i}$,

(c) if $Y \subseteq\left(\prod_{l \leq n} \lambda_{l}\right)^{n}$ is such that

$$
\left(\exists^{I} \eta_{0}\right) \ldots\left(\exists^{I} \eta_{n}\right)\left(\left\langle\eta_{0}, \ldots, \eta_{n}\right\rangle \in Y\right)
$$

then there are $\left\langle\eta_{0}^{\prime}, \ldots, \eta_{n}^{\prime}\right\rangle,\left\langle\eta_{0}^{\prime \prime}, \ldots, \eta_{n}^{\prime \prime}\right\rangle \in Y$ such that for all $l \leq n$,

$$
\mathbb{B} \models y_{\eta_{l}^{\prime}} \cap y_{\eta_{l}^{\prime \prime}}=\mathbf{0} .
$$

Proof. (a) The proof that the algebra $\mathbb{B}$ satisfies the $\theta$-Knaster condition is exactly the same as that of 3.8 (actually it is a special case).

(b) Should be clear.

(c) For $l, m \leq n$ put $\chi_{l}^{m}=\chi_{l}, \lambda_{l}^{m}=\lambda_{l}, I_{l}^{m}=I_{l}, P_{l}^{m}=\left\{\{\alpha, \beta\} \subseteq \lambda_{l}\right.$ : $\left.\mathbb{B}_{l} \models y_{\alpha}^{l} \cap y_{\beta}^{l}=\mathbf{0}\right\}, B=Y$. It is easy to check that the assumptions of Proposition 1.5 are satisfied. Applying it we find sets $X_{0}, \ldots, X_{n}$ satisfying the appropriate versions of clauses (a)-(d) there. Note that our choice of the sets $P_{l}^{m}$ and clauses (b), (c) of 1.5 imply that

$$
\begin{gathered}
X_{m}=\left\{\nu_{m}^{\prime}, \nu_{m}^{\prime \prime}\right\} \subseteq \prod_{l \leq n-m} \lambda_{l}, \quad \nu_{m}^{\prime}\left\lceil(n-m)=\nu_{m}^{\prime \prime} \uparrow(n-m),\right. \\
\mathbb{B}_{n-m} \models y_{\nu_{m}^{\prime}(n-m)}^{n-m} \cap y_{\nu_{m}^{\prime \prime}(n-m)}^{n-m}=\mathbf{0} .
\end{gathered}
$$

Look at the sequences $\left\langle\nu_{0}^{\prime}, \ldots, \nu_{n}^{\prime}\right\rangle,\left\langle\nu_{0}^{\prime \prime}, \ldots, \nu_{n}^{\prime \prime}\right\rangle$. By clause (d) of 1.5 we find $\left\langle\eta_{0}^{\prime}, \ldots, \eta_{m}^{\prime}\right\rangle,\left\langle\nu_{0}^{\prime \prime}, \ldots, \nu_{n}^{\prime \prime}\right\rangle \in Y$ such that for each $m \leq n$,

$$
\nu_{m}^{\prime} \unlhd \eta_{m}^{\prime}, \quad \nu_{m}^{\prime \prime} \unlhd \eta_{m}^{\prime \prime} .
$$

Now, the properties of $\nu_{m}^{\prime}, \nu_{m}^{\prime \prime}$ and the definition of the algebra $\mathbb{B}$ imply that for each $m \leq n$,

$$
\mathbb{B}=y_{\eta_{m}^{\prime}} \cap y_{\eta_{m}^{\prime \prime}}=\mathbf{0}
$$

Lemma 4.3. Assume that $\lambda$ is a regular cardinal, $|\delta|<\lambda, J$ is an ideal on $\delta$ extending $J_{\delta}^{\mathrm{bd}}, \mathcal{C}=\left(T, \bar{\lambda}, \bar{\eta},\left\langle\left(\mathbb{B}_{\eta}, \bar{y}_{\eta}\right): \eta \in T\right\rangle\right)$ is a $(\delta, \mu, \lambda)$-constructor and $\bar{I}$ is such that $(T, \bar{\lambda}, \bar{I}) \in \mathcal{K}_{\delta, \mu}^{\mathrm{id}}$. Suppose that $\bar{\eta}=\left\langle\eta_{\alpha}: \alpha<\lambda\right\rangle$ is a sequence stronger (or big) $J$-cofinal in $(T, \bar{\lambda}, \bar{I})$ such that

$$
(\forall i<\delta)\left(\mid\left\{\eta_{\alpha}\lceil i: \alpha<\lambda\} \mid<\lambda\right)\right. \text {. }
$$


Further, assume that

$(\ominus) \quad$ for every $n<\omega$, for a $J$-positive set of $i<\delta$ we have: if $\eta_{0}, \ldots, \eta_{n} \in$ $T_{i}$ are pairwise distinct and the set $Y \subseteq \prod_{l \leq n} \lambda_{\eta_{l}}$ is such that

$$
\left(\exists^{I_{\eta_{0}}} \gamma_{0}\right) \ldots\left(\exists^{I_{\eta_{n}}} \gamma_{n}\right)\left(\left\langle\gamma_{0}, \ldots, \gamma_{n}\right\rangle \in Y\right)
$$

then for some $\gamma_{l}^{\prime}, \gamma_{l}^{\prime \prime}<\lambda_{\eta_{l}}($ for $l \leq n)$ we have $\left\langle\gamma_{l}^{\prime}: l \leq n\right\rangle,\left\langle\gamma_{l}^{\prime \prime}: l \leq n\right\rangle$ $\in Y$ and for all $l \leq n$,

$$
\mathbb{B}_{\eta_{l}} \models y_{\eta_{l}} \frown\left\langle\gamma_{l}^{\prime}\right\rangle \cap y_{\eta_{l}} \frown\left\langle\gamma_{l}^{\prime \prime}\right\rangle=\mathbf{0} .
$$

Then the Boolean algebra $\mathbb{B}^{\text {green }}(\mathcal{C})$ satisfies the $\lambda$-cc.

Proof. Suppose that $\left\langle z_{\alpha}: \alpha<\lambda\right\rangle \subseteq \mathbb{B}^{\text {green }} \backslash\{\mathbf{0}\}$. By the standard cleaning (compare the first part of the proof of 3.8) we may assume that there are $n^{*} \in \omega$ and a function $\varepsilon: \lambda \times n^{*} \rightarrow \lambda$ such that:

(1) $z_{\alpha}=\bigcap_{l<n^{*}} x_{\varepsilon(\alpha, l)}$ (in $\left.\mathbb{B}^{\text {green }}\right)$

(2) $\varepsilon(\alpha, 0)<\varepsilon(\alpha, 1)<\ldots<\varepsilon\left(\alpha, n^{*}-1\right)$,

(3) $\left\langle\left\langle\varepsilon(\alpha, l): l<n^{*}\right\rangle: \alpha<\lambda\right\rangle$ forms a $\Delta$-system of sequences with kernel $m^{*}$, i.e., $\left(\forall l<m^{*}\right)(\varepsilon(\alpha, l)=\varepsilon(l))$ and

$$
\left(\forall l \in\left[m^{*}, n^{*}\right)\right)(\forall \alpha<\lambda)(\varepsilon(\alpha, l) \notin\{\varepsilon(\beta, k):(\beta, k) \neq(\alpha, l)\}),
$$

(4) there is $i^{*}<\delta$ such that for each $\alpha<\lambda$ there is no repetition in the sequence $\left\langle\eta_{\varepsilon(\alpha, l)}\left\lceil i^{*}: l<n^{*}\right\rangle\right.$.

Since $\mid\left\{\eta_{\alpha}\lceil i: \alpha<\lambda\} \mid<\lambda\right.$ (for $i<\delta$ ) and $|\delta|<\lambda$ we may additionally require that

( $\hat{*}) \quad$ for each $i<\delta$, for every $\alpha<\lambda$ we have

$$
\left(\exists^{\lambda} \beta<\lambda\right)\left(\forall l<n^{*}\right)\left(\eta _ { \varepsilon ( \alpha , l ) } \left\lceil(i+1)=\eta_{\varepsilon(\beta, l)}\lceil(i+1)),\right.\right.
$$

(**) for each $\alpha<\beta<\lambda, l<n^{*}$,

$$
\eta_{\varepsilon(\alpha, l)}\left\lceil i^{*}=\eta_{\varepsilon(\beta, l)}\left\lceil i^{*} .\right.\right.
$$

REMARK. Note that the claim below is like an $\left(n^{*}-m^{*}\right)$-place version of 3.7. Having an $\left(n^{*}-m^{*}\right)$-ary version is extra for the construction but it also costs.

Claim 4.3.1. Assume that: $\mathcal{C}=\left(T, \bar{\lambda}, \bar{\eta},\left\langle\left(\mathbb{B}_{\eta}, \bar{y}_{\eta}\right): \eta \in T\right\rangle\right.$ is a $(\delta, \mu, \lambda)$ constructor, $\lambda$ is a regular cardinal, $\delta<\lambda, \bar{I}$ is such that $(T, \bar{\lambda}, \bar{I}) \in \mathcal{K}_{\delta, \mu}^{\mathrm{id}}$, $J$ is an ideal on $\delta$ extending $J_{\delta}^{\mathrm{bd}}$ and the sequence $\bar{\eta}$ is stronger $J$-cofinal in $(T, \bar{\lambda}, \bar{I})$. Further suppose that $\varepsilon: \lambda \times n^{*} \rightarrow \lambda, m^{*}, n^{*}$ and $i^{*}<\delta$ are as above (after the reduction, but the property $(\hat{* *})$ is not needed). Then

( $\otimes) \quad Z_{\alpha} \in J$ for every large enough $\alpha<\lambda$, where

$$
\begin{aligned}
& Z_{\alpha}:=\left\{i<\delta: \neg\left(\exists^{I_{\left.\eta_{\varepsilon\left(\alpha, m^{*}\right.}\right)^{\uparrow i}}} \gamma_{m^{*}}\right)\left(\exists^{I_{\left.\eta_{\varepsilon\left(\alpha, m^{*}+1\right)}\right)^{\uparrow i}}} \gamma_{m^{*}+1}\right) \ldots\right. \\
& \ldots\left(\exists^{I_{\eta_{\varepsilon\left(\alpha, n^{*}-1\right)}}{ }^{\uparrow i}} \gamma_{n^{*}-1}\right)\left(\exists^{\lambda} \beta\right)\left(\forall l \in\left[m^{*}, n^{*}\right)\right)\left(\eta_{\varepsilon(\beta, l)} \uparrow(i+1)=\eta_{\varepsilon(\alpha, l)}\left\lceil i \frown\left\langle\gamma_{l}\right\rangle\right)\right\} .
\end{aligned}
$$


Proof. For $i<\delta, i \geq i^{*}$ and distinct sequences $\nu_{m^{*}}, \ldots, \nu_{n^{*}-1} \in T_{i}$ define

$$
\begin{aligned}
B_{\left\langle\nu_{l}: l \in\left[m^{*}, n^{*}\right)\right\rangle}:=\{\bar{\gamma}: & \bar{\gamma}=\left\langle\gamma_{l}: l \in\left[m^{*}, n^{*}\right)\right\rangle \text { and } \\
& \text { for arbitrarily large } \alpha<\lambda, \text { for all } m^{*} \leq l<n^{*}, \\
& \left.\nu_{l} \frown\left\langle\gamma_{l}\right\rangle \triangleleft \eta_{\varepsilon(\alpha, l)}\right\} .
\end{aligned}
$$

We will call a sequence $\left\langle\nu_{l}: l \in\left[m^{*}, n^{*}\right)\right\rangle$ a success if

$$
\left(\exists^{I_{\nu_{m}}} \gamma_{m^{*}}\right) \ldots\left(\exists^{I_{\nu^{*}-1}} \gamma_{n^{*}-1}\right)\left(\left\langle\gamma_{l}: l \in\left[m^{*}, n^{*}\right)\right\rangle \in B_{\left\langle\nu_{l} \in\left[m^{*}, n^{*}\right)\right\rangle}\right) .
$$

Using this notion we may reformulate $(\otimes)$ (which we have to prove) as

$\left(\otimes^{*}\right) \quad$ for every large enough $\alpha<\lambda$, for $J$-majority of $i<\delta, i>i^{*}$ the sequence $\left\langle\eta_{\varepsilon(\alpha, l)}\left\lceil i: l \in\left[m^{*}, n^{*}\right)\right\rangle\right.$ is a success.

To show $\left(\nabla^{*}\right)$ note that if a sequence $\left\langle\nu_{l}: l \in\left[m^{*}, n^{*}\right)\right\rangle$ is not a success then there are functions $f_{\left\langle\nu_{l}: l \in\left[m^{*}, n^{*}\right)\right\rangle}^{k}\left(\right.$ for $\left.m^{*} \leq k<n^{*}\right)$ such that

$$
f_{\left\langle\nu_{l}: l \in\left[m^{*}, n^{*}\right)\right\rangle}^{k}: \prod_{l=m^{*}}^{k-1} \lambda_{\nu_{l}} \rightarrow I_{\nu_{k}}
$$

and if $\left\langle\gamma_{l}: l \in\left[m^{*}, n^{*}\right)\right\rangle \in B_{\left\langle\nu_{l}: l \in\left[m^{*}, n^{*}\right)\right\rangle}$ then

$$
\left(\exists k \in\left[m^{*}, n^{*}\right)\right)\left(\gamma_{k} \in f_{\left\langle\nu_{l}: l \in\left[m^{*}, n^{*}\right)\right\rangle}^{k}\left(\gamma_{m^{*}}, \ldots, \gamma_{k-1}\right)\right) .
$$

If $\left\langle\nu_{l}: l \in\left[m^{*}, n^{*}\right)\right\rangle$ is a success then we declare that $f_{\left\langle\nu_{l}: l \in\left[m^{*}, n^{*}\right)\right\rangle}^{k}$ is constantly equal to $\emptyset$.

Now we may finish the proof of the claim applying clause (b) of Definition 2.2(5) to $n^{*}-1$ and functions $F_{0}, \ldots, F_{n^{*}-1}$ such that for $k \in\left[m^{*}, n^{*}\right)$, $\left.F_{k}\left(\nu_{0} \frown\left\langle\gamma_{0}\right\rangle, \ldots, \nu_{k-1} \frown\left\langle\gamma_{k-1}\right\rangle, \nu_{k}, \ldots, \nu_{n^{*}-1}\right\rangle\right)=f_{\left\langle\nu_{l}: l \in\left[m^{*}, n^{*}\right)\right\rangle}^{k}\left(\gamma_{m^{*}}, \ldots, \gamma_{k-1}\right)$. This gives us a suitable $\alpha^{*}<\lambda$. Suppose $\varepsilon\left(\alpha, m^{*}\right) \geq \alpha^{*}$. Then for $J$ majority of $i<\delta$ for each $k \in\left[m^{*}, n^{*}\right)$ we have: if

$F_{m}\left(\eta_{\varepsilon(\alpha, 0)}\left\lceil(i+1), \ldots, \eta_{\varepsilon(\alpha, k-1)}\left\lceil(i+1), \eta_{\varepsilon(\alpha, k)}\left\lceil i, \ldots, \eta_{\varepsilon\left(\alpha, n^{*}-1\right)}\lceil i) \in I_{\eta_{\varepsilon(\alpha, k) \uparrow i}}\right.\right.\right.\right.$ then

$\eta_{\varepsilon(\alpha, k)}\left\lceil(i+1) \notin F_{m}\left(\eta_{\varepsilon(\alpha, 0)}\left\lceil(i+1), \ldots, \eta_{\varepsilon(\alpha, k-1)}\left\lceil(i+1), \eta_{\varepsilon(\alpha, k)}\left\lceil i, \ldots, \eta_{\varepsilon\left(\alpha, n^{*}-1\right)}\lceil i)\right.\right.\right.\right.\right.$. But the choice of the functions $F_{k}$ implies that thus for $J$-majority of $i<\delta$, for each $k \in\left[m^{*}, n^{*}\right)$,

$$
\eta_{\varepsilon(\alpha, k)}(i) \notin f_{\left\langle\eta_{\varepsilon(\alpha, l)}^{k}\left\lceil i: l \in\left[m^{*}, n^{*}\right)\right\rangle\right.}^{k}\left(\eta_{\varepsilon\left(\alpha, m^{*}\right)}(i), \ldots, \eta_{\varepsilon(\alpha, k-1)}(i)\right) .
$$

Now the definition of the function $f_{\left\langle\nu_{l}: l \in\left[m^{*}, n^{*}\right)\right\rangle}^{k}$ works: if for some relevant $i<\delta$ above the sequence $\left\langle\eta_{\varepsilon(\alpha, l)}\left\lceil i: l \in\left[m^{*}, n^{*}\right)\right\rangle\right.$ is not a success then

$$
\left\langle\eta_{\varepsilon(\alpha, l)}(i): l \in\left[m^{*}, n^{*}\right)\right\rangle \notin B_{\left\langle\eta_{\varepsilon(\alpha, l)} \backslash i: l \in\left[m^{*}, n^{*}\right)\right\rangle},
$$

and this contradicts $(\hat{*})$ before. 
Let $\alpha^{*}$ be such that for each $\alpha \geq \alpha^{*}$ we have $Z_{\alpha} \in J$. Choose $i \in \delta \backslash Z_{\alpha^{*}}$ such that the clause $(\Theta)$ applies to $n^{*}-m^{*}$ and $i$. Let

$Y:=\left\{\left\langle\gamma_{m^{*}}, \ldots, \gamma_{n^{*}-1}\right\rangle:\left(\exists^{\lambda} \beta\right)\left(\forall l \in\left[m^{*}, n^{*}\right)\right)\left(\eta_{\varepsilon(\beta, l)} \uparrow(i+1)=\left(\eta_{\varepsilon\left(\alpha^{*}, l\right)} \uparrow i\right) \frown\left\langle\gamma_{l}\right\rangle\right)\right\}$. The definition of $Z_{\alpha^{*}}$ (and the choice of $i$ ) imply that the assumption $(\ominus$ ) applies to the set $Y$, and we get $\gamma_{l}^{\prime}, \gamma_{l}^{\prime \prime}<\lambda_{\eta_{\varepsilon\left(\alpha^{*}, l\right)} \mid i}\left(\right.$ for $\left.m^{*} \leq l<n^{*}\right)$ such that

$$
\begin{gathered}
\left\langle\gamma_{l}^{\prime}: m^{*} \leq l<n^{*}\right\rangle,\left\langle\gamma_{l}^{\prime \prime}: m^{*} \leq l<n^{*}\right\rangle \in Y, \\
\mathbb{B}_{\eta_{\varepsilon\left(\alpha^{*}, l\right)}\lceil i}=y_{\eta_{\varepsilon\left(\alpha^{*}, l\right)} \uparrow i \prec\left\langle\gamma_{l}^{\prime}\right\rangle} \cap y_{\eta_{\varepsilon\left(\alpha^{*}, l\right)}\left\lceil i \frown\left\langle\gamma_{l}^{\prime \prime}\right\rangle\right.}=\mathbf{0} \text { for } m^{*} \leq l<n^{*} .
\end{gathered}
$$

Now, choose $\alpha<\beta<\lambda$ such that for $m^{*} \leq l<n^{*}$,

$$
\eta_{\varepsilon\left(\alpha^{*}, l\right)}\left\lceil i \frown\left\langle\gamma_{l}^{\prime}\right\rangle=\eta_{\varepsilon(\alpha, l)} \uparrow(i+1), \quad \eta_{\varepsilon\left(\alpha^{*}, l\right)}\left\lceil i \frown\left\langle\gamma_{l}^{\prime \prime}\right\rangle=\eta_{\varepsilon(\beta, l)} \uparrow(i+1)\right.\right.
$$

(possible by the choice of $Y$ and $\gamma_{l}^{\prime}, \gamma_{l}^{\prime \prime}$ ). The definition of the algebra $\mathbb{B}^{\text {green }}(\mathcal{C})$ and the choice of $\gamma_{l}^{\prime}, \gamma_{l}^{\prime \prime}$ imply that for $m^{*} \leq l<n^{*}$,

$$
\mathbb{B}^{\text {green }}(\mathcal{C}) \models x_{\varepsilon(\alpha, l)} \cap x_{\varepsilon(\beta, l)} \neq \mathbf{0} \text {. }
$$

If $l \neq m$ then

$$
\mathbb{B}^{\text {green }}(\mathcal{C}) \models x_{\varepsilon(\alpha, l)} \cap x_{\varepsilon(\beta, m)} \neq \mathbf{0}
$$

by the conditions $(\hat{* *})$ and $(4)$ of the preliminary cleaning (and the definition of $\mathbb{B}^{\text {green }}(\mathcal{C})$, remember $\left.z_{\alpha} \neq \mathbf{0}\right)$. Finally, remembering that $\varepsilon(\alpha, l)=\varepsilon(\beta, l)$ for $l<m^{*}, z_{\alpha} \neq \mathbf{0}$ and $z_{\beta} \neq \mathbf{0}$, we may conclude that

$$
\mathbb{B}^{\text {green }}(\mathcal{C}) \models \bigcap_{l<n^{*}} x_{\varepsilon(\alpha, l)} \cap \bigcap_{l<n^{*}} x_{\varepsilon(\beta, l)} \neq \mathbf{0},
$$

finishing the proof of Lemma 4.3.

THEOREM 4.4. If $\mu$ is a strong limit singular cardinal, $\lambda:=2^{\mu}=\mu^{+}$ then there are Boolean algebras $\mathbb{B}_{1}, \mathbb{B}_{2}$ such that the algebra $\mathbb{B}_{1}$ satisfies the $\lambda$-cc, the algebra $\mathbb{B}_{2}$ has the $\left(2^{\mathrm{cf}(\mu)}\right)^{+}-$Knaster property but the free product $\mathbb{B}_{1} * \mathbb{B}_{2}$ does not satisfy the $\lambda$-cc.

Proof. Let $\delta=\operatorname{cf}(\mu)$ and let $h: \delta \rightarrow \omega$ be a function such that

$$
(\forall n \in \omega)\left(\exists^{\delta} i\right)(h(i)=n) .
$$

Choose an increasing sequence $\left\langle\mu_{i}: i<\delta\right\rangle$ of regular cardinals such that $\mu=\sum_{i<\delta} \mu_{i}$. Next, by induction on $i<\delta$ choose $\lambda_{i}, \chi_{i},\left(\mathbb{B}_{i}, \bar{y}_{i}\right)$ and $I_{i}$ such that:

(1) $\lambda_{i}, \chi_{i}$ are regular cardinals below $\mu$,

(2) $\lambda_{i}>\chi_{i} \geq \prod_{j<i} \lambda_{j}+\mu_{i}$

(3) $I_{i}$ is a $\chi_{i}^{+}$-complete ideal on $\lambda_{i}$ (containing all singletons),

(4) $\left(\mathbb{B}_{i}, \bar{y}_{i}\right)$ is a $\lambda_{i}$-marked Boolean algebra such that if $n=h(i)$ and the set $Y \subseteq\left(\lambda_{i}\right)^{n+1}$ is such that

$$
\left(\exists^{I_{i}} \gamma_{0}\right) \ldots\left(\exists^{I_{i}} \gamma_{n}\right)\left(\left\langle\gamma_{0}, \ldots, \gamma_{n}\right\rangle \in Y\right)
$$


then for some $\gamma_{l}^{\prime}, \gamma_{l}^{\prime \prime}<\lambda_{i}$ (for $l \leq n$ ) we have $\left\langle\gamma_{l}^{\prime}: l \leq n\right\rangle,\left\langle\gamma_{l}^{\prime \prime}: l \leq n\right\rangle \in Y$ and for all $l \leq n$,

$$
\mathbb{B}_{i}=y_{\gamma_{l}^{\prime}}^{i} \cap y_{\gamma_{l}^{\prime \prime}}^{i}=\mathbf{0},
$$

(5) each algebra $\mathbb{B}_{i}$ satisfies the $\left(2^{|\delta|}\right)^{+}$-Knaster condition.

Arriving at stage $i$ of the construction we first put $\chi_{i}=\left(\prod_{j<i} \lambda_{j}+\mu_{i}\right)^{+}$. Next we define inductively $\chi_{i, k}, \lambda_{i, k}$ for $k \leq h(i)$ such that

$$
\chi_{i, 0}=\chi_{i}, \quad \lambda_{i, k}=\left(2^{\chi_{i, k}}\right)^{+}, \quad \chi_{i, k+1}=\left(\lambda_{i, k}\right)^{+} .
$$

By 3.1, for each $k \leq h(i)$ we find a $\left(\lambda_{i, k}, \chi_{i, k}^{+}\right)$-well marked Boolean algebra $\left(\mathbb{B}_{i, k}, \bar{y}_{i, k}, I_{i, k}\right)$ such that $\mathbb{B}_{i, k}$ has the $\left(2^{\delta}\right)^{+}$-Knaster property (compare 3.3 ). Let $\lambda_{i}=\lambda_{i, h(i)}$. Proposition 4.2 applied to $\left\langle\left(\mathbb{B}_{i, k}, \bar{y}_{i, k}, I_{i, k}\right): k \leq h(i)\right\rangle$ provides a $\lambda_{i}$-marked Boolean algebra $\left(\mathbb{B}_{i}, \bar{y}_{i}\right)$ and a $\chi_{i}^{+}$-complete ideal $I_{i}$ on $\lambda_{i}$ such that the requirements (4), (5) above are satisfied.

Now put $T=\bigcup_{j<\delta} \prod_{i<j} \lambda_{i}$ and for $\eta \in T$,

$$
\mathbb{B}_{\eta}=\mathbb{B}_{\lg (\eta)}, \quad \bar{y}_{\eta}=\bar{y}_{\lg (\eta)}, \quad I_{\eta}=I_{\lg (\eta)} .
$$

By 2.8 we find a stronger $J_{\delta}^{\mathrm{bd}}$-cofinal sequence $\bar{\eta}=\left\langle\eta_{\alpha}: \alpha<\lambda\right\rangle$ for $(T, \bar{\lambda}, \bar{I})$. Take the $\left(\delta, \mu, \mu^{+}\right)$-constructor $\mathcal{C}$ determined by these parameters. Look at the algebras $\mathbb{B}_{2}=\mathbb{B}^{\text {red }}(\mathcal{C}), \mathbb{B}_{1}=\mathbb{B}^{\text {green }}(\mathcal{C})$. Applying 4.1 we see that $\mathbb{B}_{1} * \mathbb{B}_{2}$ fails the $\lambda$-cc. The choice of the function $h$ and the requirement (4) above allow us to apply 4.3 to conclude that the algebra $\mathbb{B}_{2}$ satisfies the $\lambda$-cc. Finally, by 3.8 , we conclude that $\mathbb{B}_{1}$ has the $\left(2^{\delta}\right)^{+}$-Knaster property.

REMARK 4.5. (1) We shall later give results not using $2^{\mu}=\mu^{+}$but still not in ZFC.

(2) Applying the methods of [1] or [3] we hope to prove the consistency of: for some $\mu$ strong limit singular there is no example for $\lambda=\mu^{+}$.

(3) If we want "for no regular $\lambda \in\left[\mu, 2^{\mu}\right]$ " more is needed; we expect the consistency, but it is harder (not to speak of "for all $\mu$ ")

(4) Remark (1) above shows that $2^{\mu}>\mu^{+}$is not enough for the negative result.

\section{Toward improvements}

Definition 5.1. Let $(T, \bar{\lambda}, \bar{I}) \in \mathcal{K}_{\mu, \delta}^{\mathrm{id}}$ and let $J$ be an ideal on $\delta$ (including $J_{\delta}^{\mathrm{bd}}$, as usual). We say that a sequence $\bar{\eta}=\left\langle\eta_{\alpha}: \alpha<\lambda\right\rangle$ of $\delta$-branches through $T$ is super $J$-cofinal for $(T, \bar{\lambda}, \bar{I})$ if

(a) $\eta_{\alpha} \neq \eta_{\beta}$ for distinct $\alpha, \beta<\lambda$,

(b) for every function $F$ there is $\alpha^{*}<\lambda$ such that if $\alpha_{0}<\ldots<\alpha_{n}<\lambda$, $\alpha^{*} \leq \alpha_{n}$ then the set 


$$
\begin{aligned}
\{i<\delta: & (\text { ii })^{*} F\left(\eta_{\alpha_{0}}, \ldots, \eta_{\alpha_{n-1}}, \eta_{\alpha_{n}}\lceil i) \in I_{\eta_{\alpha_{n}} \backslash i}\right. \\
& \text { (and well defined) but } \\
& \eta_{\alpha_{n}}\left\lceil(i+1) \in F\left(\eta_{\alpha_{0}}, \ldots, \eta_{\alpha_{n-1}}, \eta_{\alpha_{n}}\lceil i)\right\}\right.
\end{aligned}
$$

is in the ideal $J$.

Remark 5.2. (1) The main difference between the definition of super $J$-cofinal sequence and those in 2.2 is the fact that here the values of the function $F$ depend on $\eta_{\alpha_{l}}$ (for $l<n$ ), not on the restrictions of these sequences as in earlier notions.

(2) "Super* $J$-cofinal" is defined by adding " $\alpha * \leq \alpha_{0}$ " (compare 2.2(9)).

Proposition 5.3. Suppose that $(T, \bar{\lambda}, \bar{I}) \in \mathcal{K}_{\mu, \delta}^{\mathrm{id}}$ is such that for each $\nu \in T_{i}, i<\delta$ the ideal $I_{\nu}$ is $\left|T_{i}\right|^{+}$-complete. Let $J \supseteq J_{\delta}^{\text {bd }}$ be an ideal on $\delta$. Then every super $J$-cofinal sequence is stronger* $J$-cofinal.

Proof. Assume that $\bar{\eta}=\left\langle\eta_{\alpha}: \alpha<\lambda\right\rangle \subseteq \lim _{\delta}(T)$ is super $J$-cofinal for $(T, \bar{\lambda}, \bar{I})$. Let $n<\omega$ and let $F_{0}, \ldots, F_{n-1}$ be functions. For each $l \leq n$ we define an $(l+1)$-place function $F_{l}^{*}$ such that if $\alpha_{0}<\alpha_{1}<\ldots<\alpha_{l-1}<\lambda$, $\varrho \in T_{i}, i<\delta$ then

$$
\begin{aligned}
F_{l}^{*} & \left(\eta_{\alpha_{0}}, \ldots, \eta_{\alpha_{l-1}}, \varrho\right) \\
= & \bigcup\left\{F_{l}\left(\eta_{\alpha_{0}} \uparrow(i+1), \ldots, \eta_{\alpha_{l-1}} \uparrow(i+1), \varrho, \nu_{l+1}, \ldots, \nu_{n}\right): \nu_{l+1}, \ldots, \nu_{n} \in T_{i} \&\right. \\
& F_{l}\left(\eta_{\alpha_{0}} \uparrow(i+1), \ldots, \eta_{\alpha_{l-1}}\left\lceil(i+1), \varrho, \nu_{l+1}, \ldots, \nu_{n}\right) \in I_{\varrho} \text { (and well defined) }\right\} .
\end{aligned}
$$

As the ideals $I_{\varrho}$ (for $\varrho \in T_{i}$ ) are $\left|T_{i}\right|^{+}$-complete we know that

$$
F_{l}^{*}\left(\eta_{\alpha_{0}}, \ldots, \eta_{\alpha_{l-1}}, \varrho\right) \in I_{\varrho}
$$

Applying 5.1(b) to the functions $F_{l}^{*}(l<n)$ we choose $\alpha_{l}^{*}<\lambda$ such that if $\alpha_{0}<\ldots<\alpha_{l}<\lambda, \alpha_{l}^{*} \leq \alpha_{l}$ then the set

is in the ideal $J$.

$$
\begin{aligned}
B_{l}^{*}:=\{i<\delta: & F_{l}^{*}\left(\eta_{\alpha_{0}}, \ldots, \eta_{\alpha_{l-1}}, \eta_{\alpha_{l}}\lceil i) \in I_{\eta_{\alpha_{l}} \uparrow i}\right. \text { but } \\
& \left.\eta_{\alpha_{l}} \uparrow(i+1) \in F_{l}^{*}\left(\eta_{\alpha_{0}}, \ldots, \eta_{\alpha_{l-1}}, \eta_{\alpha_{l}} \uparrow i\right)\right\}
\end{aligned}
$$

Put $\alpha^{*}=\max \left\{\alpha_{l}^{*}: l \leq n\right\}$. We want to show that this $\alpha^{*}$ works for the condition 2.2(6)(b) (version for "stronger*"). So suppose that $m \leq n$, $\alpha^{*} \leq \alpha_{0}<\alpha_{1}<\ldots<\alpha_{n}<\lambda$. Let

$$
\begin{aligned}
B_{m}:= & \left\{i<\delta: F_{m}\left(\eta_{\alpha_{0}} \uparrow(i+1), \ldots, \eta_{\alpha_{m-1}} \uparrow(i+1), \eta_{\alpha_{m}}\left\lceil i, \ldots, \eta_{\alpha_{n}}\lceil i) \in I_{\eta_{\alpha_{m}}\lceil i}\right.\right.\right. \\
& \text { and } \eta_{\alpha_{m}} \uparrow(i+1) \in F_{m}\left(\eta_{\alpha_{0}} \uparrow(i+1), \ldots, \eta_{\alpha_{m-1}} \uparrow(i+1), \eta_{\alpha_{m}}\left\lceil i, \ldots, \eta_{\alpha_{n}}\lceil i)\right\} .\right.
\end{aligned}
$$

Note that if $i \in B_{m}$ then, as $\alpha_{m}^{*} \leq \alpha^{*} \leq \alpha_{m}$,

$$
\begin{aligned}
\eta_{\alpha_{m}} \uparrow(i+1) & \in F_{m}\left(\eta_{\alpha_{0}} \uparrow(i+1), \ldots, \eta_{\alpha_{m-1}} \uparrow(i+1), \eta_{\alpha_{m}}\left\lceil i, \ldots, \eta_{\alpha_{n}}\lceil i)\right.\right. \\
& \subseteq F_{m}^{*}\left(\eta_{\alpha_{0}}, \ldots, \eta_{\alpha_{m-1}}, \eta_{\alpha_{m}} \uparrow i\right) \in I_{\eta_{\alpha_{m}} \uparrow i} .
\end{aligned}
$$

Hence we conclude that $B_{m} \subseteq B_{m}^{*}$ and therefore $B_{m} \in J$. 
Proposition 5.4. Assume that $(T, \bar{\lambda}, \bar{I}) \in \mathcal{K}_{\mu, \delta}^{\mathrm{id}}$, each ideal $I_{\eta}($ for $\eta \in$ $\left.T_{i}, i<\delta\right)$ is $\left(|\delta|+\left|T_{i}\right|\right)^{+}$-complete and $J \supseteq J_{\delta}^{\mathrm{bd}}$ is an ideal on $\delta$. Further suppose that a sequence $\bar{\eta}=\left\langle\eta_{\alpha}: \alpha<\lambda\right\rangle$ is super $J$-cofinal for $(T, \bar{\lambda}, \bar{I}), \lambda$ is a regular cardinal greater than $|T|$ and a sequence $\left\langle\alpha_{\varepsilon, l}: \varepsilon<\lambda, l<n\right\rangle \subseteq \lambda$ is with no repetition and such that

$$
\alpha_{\varepsilon, 0}<\alpha_{\varepsilon, 1}<\ldots<\alpha_{\varepsilon, n-1} \quad \text { for all } \varepsilon<\lambda .
$$

Then for every $\varepsilon<\lambda$ large enough there is a $\in J$ such that

$(\odot) \quad$ if $i_{l} \in \delta \backslash a($ for $l<n)$ and $i_{0} \geq i_{1} \geq \ldots \geq i_{n-1}$ then

$$
\begin{aligned}
& \left(\exists^{I_{\eta_{\varepsilon, 0} \vdash i_{0}}} \gamma_{0}\right) \ldots\left(\exists^{I_{\eta_{\alpha_{\varepsilon, n-1}} i_{n-1}}} \gamma_{n-1}\right) \\
& \left(\exists^{\lambda} \zeta<\lambda\right)(\forall l<n)\left(\eta_{\alpha_{\zeta, l}} l\left(i_{l}+1\right)=\eta_{\alpha_{\varepsilon, l} l}\left\lceil i_{l} \frown\left\langle\gamma_{l}\right\rangle\right) .\right.
\end{aligned}
$$

Proof. This is very similar to Claim 4.3.1. First choose $\varepsilon_{0}<\lambda$ such that for each $\varepsilon \in\left[\varepsilon_{0}, \lambda\right)$ and for every $i_{0}, \ldots, i_{n-1}<\delta$ we have

$$
\left(\exists^{\lambda} \zeta<\lambda\right)(\forall l<n)\left(\eta _ { \alpha _ { \zeta , l } } \left\lceil\left(i_{l}+1\right)=\eta_{\alpha_{\varepsilon, l}}\left\lceil\left(i_{l}+1\right)\right)\right.\right.
$$

(possible as $|T|<\operatorname{cf}(\lambda)=\lambda$ ).

Now, for $\bar{\imath}=\left\langle i_{l}: l<n\right\rangle \subseteq \delta$ and $\bar{\nu}=\left\langle\nu_{l}: l<n\right\rangle$ such that $i_{0} \geq i_{1} \geq$ $\ldots \geq i_{n-1}, \nu_{l} \in T_{i_{l}}$ and $k<n$ we define a function $f_{\bar{\imath}, \bar{\nu}}^{k}: \prod_{l<k} \lambda_{\nu_{l}} \rightarrow I_{\nu_{k}}$ (with the convention that $f_{\bar{\imath}, \bar{\nu}}^{0}$ is supposed to be a 0-place function, i.e., a constant) as follows.

Let

$$
B_{\bar{\imath}, \bar{\nu}}:=\left\{\left\langle\gamma_{l}: l<n\right\rangle \in \prod_{l<n} \lambda_{\nu_{l}}:\left(\exists^{\lambda} \zeta<\lambda\right)(\forall l<n)\left(\eta_{\alpha_{\zeta, l}}\left\lceil\left(i_{l}+1\right)=\nu_{l} \frown\left\langle\gamma_{l}\right\rangle\right)\right\} .\right.
$$

If

$\left(\diamond_{\bar{\imath}, \bar{\nu}}\right) \quad \neg\left(\exists^{I_{\nu_{0}}} \gamma_{0}\right) \ldots\left(\exists^{I_{\nu_{n-1}}} \gamma_{n-1}\right)\left(\left\langle\gamma_{0}, \ldots, \gamma_{n-1}\right\rangle \in B_{\bar{\imath}, \bar{\nu}}\right)$

then $f_{\bar{i}, \bar{\nu}}^{0}, \ldots, f_{\bar{\imath}, \bar{\nu}}^{n-1}$ are such that

$(\diamond) \quad$ if $\left\langle\gamma_{0}, \ldots, \gamma_{n-1}\right\rangle \in B_{\bar{l}, \bar{\nu}}$ then $(\exists k<n)\left(\gamma_{k} \in f_{\bar{l}, \bar{\nu}}^{k}\left(\gamma_{0}, \ldots, \gamma_{k-1}\right)\right)$.

Otherwise (i.e., if not $\left.\left(\boldsymbol{\nu}_{\bar{i}, \bar{\nu}}\right)\right)$ the functions $f_{\bar{i}, \bar{\nu}}^{k}$ are constantly equal to $\emptyset$ (for $k<n)$. Next, for $k<n$, choose functions $F_{k}$ such that if $\eta_{0}, \ldots, \eta_{k} \in$ $\lim _{\delta}(T), i<\delta$ then

$$
\begin{aligned}
& F_{k}\left(\eta_{0}, \ldots, \eta_{k-1}, \eta_{k} \backslash i\right) \\
& =\bigcup\left\{f_{\bar{\imath}, \bar{\nu}}^{k}\left(\eta_{0}\left(i_{0}\right), \ldots, \eta_{k-1}\left(i_{k-1}\right)\right): \bar{\imath}=\left\langle i_{l}: l<n\right\rangle, \bar{\nu}=\left\langle\nu_{l}: l<n\right\rangle,\right. \\
& \delta>i_{0} \geq \ldots \geq i_{k}=i \geq i_{k+1} \geq \ldots \geq i_{n-1}, \\
& \nu_{l}=\eta_{l} \mid i_{l} \text { for } l \leq k \text { and } \\
& \left.\nu_{l} \in T_{i_{l}} \text { for } k<l<n\right\} \text {. }
\end{aligned}
$$


Note that $F_{k}\left(\eta_{0}, \ldots, \eta_{k-1}, \eta_{k} \backslash i\right)$ is a union of at most $|\delta|+\left|T_{i}\right|$ sets from the ideal $I_{\eta_{k} \uparrow i}$ and hence $F_{k}\left(\eta_{0}, \ldots, \eta_{k-1}, \eta_{k}\lceil i) \in I_{\eta_{k} \uparrow i}\right.$ (for each $\eta_{0}, \ldots, \eta_{k} \in$ $\left.\lim _{\delta}(T), i<\delta\right)$. Thus, using the super $J$-cofinality of $\bar{\eta}$ we find $\alpha^{*}<\lambda$ such that if $\alpha^{*} \leq \alpha_{0}<\ldots<\alpha_{n}<\lambda$ then the set

$$
\left\{i<\delta:(\exists k<n)\left(\eta_{\alpha_{k}}(i) \in F_{k}\left(\eta_{\alpha_{0}}, \ldots, \eta_{\alpha_{k-1}}, \eta_{\alpha_{k}}\right)\right)\right\}
$$

is in the ideal $J$.

Let $\varepsilon_{1}>\varepsilon_{0}$ be such that for every $\varepsilon \in\left[\varepsilon_{1}, \lambda\right)$ we have $\alpha^{*}<\alpha_{\varepsilon, 0}<\ldots<$ $\alpha_{\varepsilon, n-1}$.

Suppose now that $\varepsilon_{1}<\varepsilon<\lambda$. By the choice of $\alpha^{*}$ we know that the set

$$
a:=\left\{i<\delta:(\exists l<n)\left(\eta_{\alpha_{\varepsilon, l}}(i) \in F_{l}\left(\eta_{\alpha_{\varepsilon, 0}}, \ldots \eta_{\alpha_{\varepsilon, l-1}}, \eta_{\alpha_{\varepsilon, l}}\lceil i)\right)\right\}\right.
$$

is in the ideal $J$. We are going to show that the assertion $(\bullet)$ holds for $\varepsilon$ and $a$.

Suppose that $\bar{\imath}=\left\langle i_{l}: l<n\right\rangle \subseteq \delta \backslash a$ and $i_{0} \geq i_{1} \geq \ldots \geq i_{n-1}$. Let $\bar{\nu}=\left\langle\nu_{l}: l<n\right\rangle, \nu_{l}=\eta_{\alpha_{\varepsilon, l}} \mid i_{l}$. If the condition $\left(\boldsymbol{\nabla}_{\bar{i}, \bar{\nu}}\right)$ fails then we are done. So assume that it holds true. By the choice of the set $a$ (and $\alpha^{*}$ ) we have

$$
(\forall l<n)\left(\eta_{\alpha_{\varepsilon, l}}\left(i_{l}\right) \notin F_{l}\left(\eta_{\alpha_{\varepsilon, 0}}, \ldots, \eta_{\alpha_{\varepsilon, l-1}}, \eta_{\alpha_{\varepsilon, l}} \backslash i_{l}\right)\right),
$$

which, by the definition of $F_{l}$, implies that

$$
(\forall l<n)\left(\eta_{\alpha_{\varepsilon, l}}\left(i_{l}\right) \notin f_{\bar{\imath}, \bar{\nu}}^{l}\left(\eta_{\alpha_{\varepsilon, 0}}\left(i_{0}\right), \ldots, \eta_{\alpha_{\varepsilon, l-1}}\left(i_{l-1}\right)\right)\right) .
$$

By $(\diamond)$ we conclude that

$$
\left\langle\eta_{\alpha_{\varepsilon, 0}}\left(i_{0}\right), \ldots, \eta_{\alpha_{\varepsilon, n-1}}\left(i_{n-1}\right)\right\rangle \notin B_{\bar{\imath}, \bar{\nu}},
$$

and hence, by the definition of $B_{\bar{\imath}, \bar{\nu}}$,

$$
\neg\left(\exists^{\lambda} \zeta\right)(\forall l<n)\left(\eta_{\alpha_{\zeta, l}} \uparrow\left(i_{l}+1\right)=\eta_{\alpha_{\varepsilon, l}} \uparrow\left(i_{l}\right)\right),
$$

which contradicts the choice of $\varepsilon_{0}$ (remember $\varepsilon \geq \varepsilon_{1}>\varepsilon_{0}$ ).

Definition 5.5. We say that a $\lambda$-marked Boolean algebra $(\mathbb{B}, \bar{y})$ has character $n$ if for every finite set $u \in[\lambda]^{<\omega}$ such that $\mathbb{B} \models \bigcap_{\alpha \in u} y_{\alpha}=\mathbf{0}$ there exists a subset $v \subseteq u$ of size $|v| \leq n$ such that $\mathbb{B} \models \bigcap_{\alpha \in v} y_{\alpha}=\mathbf{0}$.

Proposition 5.6. If a $\lambda$-marked Boolean algebra $(\mathbb{B}, \bar{y})$ is $(\theta, \operatorname{not} \lambda)$ Knaster (or other examples considered in the present paper) and $(\mathbb{B}, \bar{y})$ has character 2 then without loss of generality $(\mathbb{B}, \bar{y})$ is determined by a colouring on $\lambda:$ if $c:[\lambda]^{2} \rightarrow 2$ is such that

$$
c(\{\alpha, \beta\})=0 \quad \text { iff } \quad \mathbb{B}=y_{\alpha} \cap y_{\beta}=\mathbf{0}
$$

then the algebra $\mathbb{B}$ is freely generated by $\left\{y_{\alpha}: \alpha<\lambda\right\}$ except that

$$
\text { if } c(\{\alpha, \beta\})=0 \text { then } y_{\alpha} \cap y_{\beta}=0 \text {. }
$$

REMARK 5.7. These are nice examples. 
PROPOSITION 5.8. In all our results (like 3.1 or 3.8 ), the marked Boolean algebra $(\mathbb{B}, \bar{y})$ which we get is actually of character 2 as long as any $\left(\mathbb{B}_{\eta}, \bar{y}_{\eta}\right)$ appearing in the assumptions (if any) is like that. Then automatically the $\theta$-Knaster property of the marked Boolean algebra $(\mathbb{B}, \bar{y})$ implies a stronger condition: if $Z \in[\lg (\bar{y})]^{\theta}$ then there is a set $Y \in[Z]^{\theta}$ such that $\left\{y_{i}: i \in Y\right\}$ generates a filter in $\mathbb{B}$.

Proposition 5.9. Let $(T, \bar{\lambda}, \bar{I}) \in \mathcal{K}_{\mu, \delta}^{\mathrm{id}}$ be such that for each $\eta \in T$ the filter $\left(I_{\eta}\right)^{c}$ (dual to $\left.I_{\eta}\right)$ is an ultrafilter on $\operatorname{succ}_{T}(\eta)$, and let $J$ be an ideal on $\delta$ (extending $\left.J_{\delta}^{\mathrm{bd}}\right)$. Suppose that:

(a) $\mathcal{C}=\left(T, \bar{\lambda}, \bar{\eta},\left\langle\left(\mathbb{B}_{\eta}, \bar{y}_{\eta}\right): \eta \in T\right\rangle\right)$ is a $(\delta, \mu, \lambda)$-constructor and the sequence $\bar{\eta}$ is stronger $J$-cofinal for $(T, \bar{\lambda}, \bar{I}),|T|<\operatorname{cf}(\lambda)=\lambda$,

(b) the sequence $\left\langle\alpha_{\varepsilon, l}: \varepsilon<\lambda, l<n\right\rangle \subseteq \lambda$ is with no repetition,

(c) for any distinct $\eta, \nu \in T$ either the ideal $I_{\eta}$ is $\left(2^{\lambda_{\nu}}\right)^{+}$-complete (which, of course, implies $\left.\lambda_{\eta}>2^{\lambda_{\nu}}\right)$ or the ideal $I_{\nu}$ is $\left(2^{\lambda_{\eta}}\right)^{+}$-complete (it is enough if this holds true for $\eta, \nu$ such that $\lg (\eta)=\lg (\nu))$.

Then for every large enough $\varepsilon<\lambda$, for $J$-almost all $i<\delta$ there are sets $X_{l} \in\left(I_{\eta_{\alpha_{\varepsilon}, l} i}\right)^{+}($for $l<n)$ such that

$$
\left(\forall \gamma_{0} \in X_{0}\right) \ldots\left(\forall \gamma_{n-1} \in X_{n-1}\right)\left(\exists^{\lambda} \zeta<\lambda\right)(\forall l<n)\left(\eta_{\alpha_{\varepsilon, l}}\left\lceil i \frown\left\langle\gamma_{l}\right\rangle \triangleleft \eta_{\alpha_{\zeta, l}}\right) .\right.
$$

REMARK 5.9.A. We can replace stronger by big and then omit being an ultrafilter.

Pr o of (of Proposition 5.9). First note that we may slightly reindex our sequence $\left\langle\alpha_{\varepsilon, l}: \varepsilon<\lambda, l<n\right\rangle$ and assume that for each $\varepsilon<\lambda$,

$$
\alpha_{\varepsilon, 0}<\alpha_{\varepsilon, 1}<\ldots<\alpha_{\varepsilon, n-1} .
$$

Now, since $|T|<\operatorname{cf}(\lambda)=\lambda$ we may apply Claim 4.3.1 to

$$
\left\langle\left\langle\alpha_{\varepsilon, l}: l<n\right\rangle: \varepsilon_{0} \leq \varepsilon<\lambda\right\rangle
$$

(we need to take $\varepsilon_{0}$ large enough to get the condition $(\hat{*})$ of the proof of 4.3). Consequently, we may conclude that there is $\varepsilon_{1}<\lambda$ such that for every $\varepsilon \in\left[\varepsilon_{1}, \lambda\right)$,

$\left(\nabla_{\varepsilon}\right)$ for $J$-majority of $i<\delta$ we have

$$
\left(\exists^{I_{\alpha_{\varepsilon, 0} \uparrow i}} \gamma_{0}\right) \ldots\left(\exists^{I_{\alpha_{\varepsilon, n-1}{ }^{i i}}} \gamma_{n-1}\right)\left(\exists^{\lambda} \zeta<\lambda\right)(\forall l<n)\left(\eta _ { \alpha _ { \zeta , l } } \left\lceil(i+1)=\eta_{\alpha_{\varepsilon, l}}\left\lceil i \frown\left\langle\gamma_{l}\right\rangle\right) .\right.\right.
$$

Now we would like to apply 1.2. We cannot do this directly as we do not know if the cardinals $\lambda_{\eta_{\varepsilon, l} \uparrow i}$ are decreasing (with $l$ ). However the following claim helps us. 
Claim 5.9.1. Suppose that $\lambda_{0}<\lambda_{1}$ are cardinals and $I_{0}, I_{1}$ are maximal ideals on $\lambda_{0}, \lambda_{1}$ respectively. Assume that the ideal $I_{1}$ is $\left(\lambda_{0}\right)^{+}$-complete and $\varphi(x, y)$ is a formula. Then

$$
\left(\exists^{I_{0}} \gamma_{0}\right)\left(\exists^{I_{1}} \gamma_{1}\right) \varphi\left(\gamma_{0}, \gamma_{1}\right) \Rightarrow\left(\exists^{I_{1}} \gamma_{1}\right)\left(\exists^{I_{0}} \gamma_{0}\right) \varphi\left(\gamma_{0}, \gamma_{1}\right) .
$$

Proof. First note that if $I$ is a maximal ideal then the quantifiers $\exists^{I}$ and $\forall^{I}$ are equivalent. Suppose now that

$$
\left(\exists^{I_{0}} \gamma_{0}\right)\left(\exists^{I_{1}} \gamma_{1}\right) \varphi\left(\gamma_{0}, \gamma_{1}\right) \text {. }
$$

This implies (as $I_{0}, I_{1}$ are maximal) that

$$
\left(\forall^{I_{0}} \gamma_{0}\right)\left(\forall^{I_{1}} \gamma_{1}\right) \varphi\left(\gamma_{0}, \gamma_{1}\right)
$$

Thus we have a set $a \in I_{0}$ and for each $\gamma \in \lambda_{0} \backslash a$ a set $b_{\gamma} \in I_{1}$ such that

$$
\left(\forall \gamma_{0} \in \lambda_{0} \backslash a\right)\left(\forall \gamma_{1} \in \lambda_{1} \backslash b_{\gamma_{0}}\right) \varphi\left(\gamma_{0}, \gamma_{1}\right) .
$$

Let $b=\bigcup_{\gamma \in \lambda_{0} \backslash a} b_{\gamma}$. As $I_{1}$ is $\left(\lambda_{0}\right)^{+}$-complete the set $b$ is in $I_{1}$. Clearly

$$
\left(\forall \gamma_{1} \in \lambda_{1} \backslash b\right)\left(\forall \gamma_{0} \in \lambda \backslash a\right) \varphi\left(\gamma_{0}, \gamma_{1}\right),
$$

which implies $\left(\exists^{I_{1}} \gamma_{1}\right)\left(\exists^{I_{0}} \gamma_{0}\right) \varphi\left(\gamma_{0}, \gamma_{1}\right)$.

Now fix $\varepsilon>\varepsilon_{1}$ ( $\varepsilon_{1}$ as chosen earlier). Take $i^{*}<\delta$ such that the elements of $\left\langle\eta_{\alpha_{\varepsilon, l}} \mid i: l<n\right\rangle$ are pairwise distinct. Suppose that $i \in\left[i^{*}, \delta\right)$ is such that the formula of $\left(\nabla_{\varepsilon}\right)$ holds true. Let $\left\{k_{l}: l<n\right\}$ be an enumeration of $n$ such that

$$
\lambda_{\eta_{\alpha_{\varepsilon, k_{0}}}\lceil i}>\lambda_{\eta_{\alpha_{\varepsilon, k_{1}}} \uparrow i}>\ldots>\lambda_{\eta_{\alpha_{\varepsilon}, k_{n-1}} \nmid i} .
$$

(Note that by the assumption (c) we know that all the $\lambda_{\eta_{\alpha_{\varepsilon, k_{l}}}}{ }_{i}$ are distinct, remember the choice of $i^{*}$.) Applying Claim 5.9.1 we conclude that

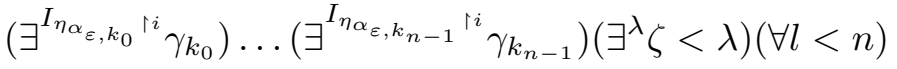

$$
\begin{aligned}
& \left(\eta _ { \alpha _ { \varepsilon , l } } \left\lceili \curlyvee\left\langle\gamma_{l}\right\rangle=\eta_{\alpha_{\zeta, l}}\lceil(i+1)) .\right.\right.
\end{aligned}
$$

But now we are able to use 1.2 to find that there are sets $X_{k_{l}} \subseteq \lambda_{\eta_{\alpha_{\varepsilon, k_{l}}} \mid i}$, $X_{k_{l}} \notin I_{\eta_{\alpha_{\varepsilon, k_{l}}} \backslash i}($ for $l<n)$ such that

$$
\prod_{l<n} X_{l} \subseteq\left\{\left\langle\gamma_{0}, \ldots, \gamma_{n-1}\right\rangle:\left(\exists^{\lambda} \zeta<\lambda\right)(\forall l<n)\left(\eta_{\alpha_{\varepsilon, l}}\left\lceil i \curlyvee\left\langle\gamma_{l}\right\rangle=\eta_{\alpha_{\zeta, l}}\lceil(i+1))\right\},\right.\right.
$$

which is exactly what we need.

If we assume less completeness of the ideals $I_{\eta}$ in 5.9 then still we may say something.

Proposition 5.10. Let $\left\langle\sigma_{i}: i<\delta\right\rangle$ be a sequence of cardinals. Suppose that $T, \bar{\lambda}, \bar{I}, \bar{\eta}, J, \lambda, \mu, \delta$ and $\left\langle\alpha_{\varepsilon, l}: \varepsilon<\lambda, l<n\right\rangle$ are as in 5.9 but with condition (c) replaced by 
(c) $_{\left\langle\sigma_{i}: i<\delta\right\rangle}^{-}$if $\eta, \nu \in T_{i}, \eta \neq \nu, i<\delta$ then either the ideal $I_{\eta}$ is $\left(\left(\lambda_{\nu}\right)^{\sigma_{i}}\right)^{+}$complete or the ideal $I_{\nu}$ is $\left(\left(\lambda_{\eta}\right)^{\sigma_{i}}\right)^{+}$-complete.

Then for every large enough $\varepsilon<\lambda$, for $J$-almost all $i<\delta$ there are sets $X_{l} \in\left[\lambda_{\eta_{\alpha_{\varepsilon}, l} \mid i}\right]^{\sigma_{i}}($ for $l<n)$ such that

$$
\left(\forall \gamma_{0} \in X_{0}\right) \ldots\left(\forall \gamma_{n-1} X_{n-1}\right)\left(\exists^{\lambda} \zeta<\lambda\right)(\forall l<n)\left(\eta_{\alpha_{\varepsilon, l}}\left\lceil i \curlyvee\left\langle\gamma_{l}\right\rangle \triangleleft \eta_{\alpha_{\zeta, l}}\right) .\right.
$$

Proof. The proof goes exactly as the one of 5.9, but instead of 1.2 we use 1.3.

REMARK 5.11. (1) Note that in the situation as in 5.9, usually " $J$ cofinal" implies "stronger $J$-cofinal" (see 2.7, 2.5).

(2) The first assumption of 5.9 (ultrafilters) coupled with our normal completeness demands is a very heavy condition, but it has rewards.

(3) A natural context here is when $\left\langle\mu_{i}: i \leq \kappa\right\rangle$ is a strictly increasing continuous sequence of cardinals such that each $\mu_{i+1}$ is compact and $\mu=$ $\mu_{\kappa}$. Then every $\mu_{i+1}$-complete filter can be extended to an $\mu_{i+1}$-complete ultrafilter. Moreover $2^{\mu}=\mu^{+}$follows by Solovay [26].

If for some function $f$ from cardinals to cardinals and for each $\chi$ there is an algebra $\mathbb{B}_{\chi}$ of cardinality $f(\chi)$ which cannot be decomposed into $\leq \mu$ sets $X_{i}$ each with some property $\operatorname{Pr}\left(\mathbb{B}_{\chi}, X_{i}\right)$ and if each $\mu_{i}$ is $f$-inaccessible then we can find $T, \bar{I}, \bar{\lambda}$ as in 5.9 and such that $\eta \in T_{i} \Rightarrow \mu_{i}<\chi_{\eta}<\lambda_{\eta}<\mu_{i+1}$ and for $\eta \in T_{i}$ there is an algebra $\mathbb{B}_{\eta}$ with universe $\lambda_{\eta}$ and the ideal $I_{\eta}$ is $\chi_{\eta}$-complete,

$$
\text { if } X \subseteq \mathbb{B}_{\eta} \text { and } \operatorname{Pr}\left(\mathbb{B}_{\eta}, X\right) \text { then } X \in I_{\eta}
$$

(compare 3.1) and $\lambda_{\eta}<\lambda_{\nu} \Rightarrow\left(2^{\lambda_{\eta}}\right)^{+}<\chi_{\nu}$. Now choosing cofinal $\bar{\eta}$ we may proceed as in earlier arguments.

(4) It seems to be good for building nice examples, however we did not find the right question yet.

(5) Central to our proofs is the assumption that

$$
\text { " }\left\langle\alpha_{\zeta, l}: \zeta<\lambda, l<n\right\rangle \subseteq \lambda \text { is a sequence with no repetition", }
$$

i.e., we deal with $\lambda$ disjoint $n$-tuples. This is natural as the examples constructed here are generated from $\left\{x_{i}: i<\lambda\right\}$ by finitary functions. One may ask what happens if we admit functions with, say, $\aleph_{0}$ places. We can still try to deduce for $\mu$ as above that:

$(\otimes)$ there is $h:\left[\mu^{+}\right]^{2} \rightarrow 2$ such that if $\left\langle u_{\varepsilon}: \varepsilon<\lambda\right\rangle$ are pairwise disjoint, $u_{\varepsilon}=\left\{\alpha_{\varepsilon, l}: l<l^{*}\right\}$ is the increasing (with $l$ ) enumeration, $l^{*}<\mu$ ( $l^{*}$ infinite), for a sequence $\left\langle\nu_{l}: l<l^{*}\right\rangle \subseteq T_{i}$ we set

$$
\begin{aligned}
& B_{\left\langle\nu_{l}: l<l^{*}\right\rangle}:= \\
& \left\{\left\langle\eta_{\alpha_{\varepsilon, l}}(i): l<l^{*}\right\rangle:\left(\exists^{\lambda} \zeta<\lambda\right)\left(\forall l<l^{*}\right)\left(\eta_{\alpha_{\varepsilon, l}}\left\lceil(i+1)=\eta_{\alpha_{\zeta, l}} l(i+1)\right)\right\},\right.
\end{aligned}
$$


for some $i^{*}<\delta$ there are no repetitions in $\left\langle\eta_{\alpha_{\varepsilon, l}} \mid i^{*}: l<l^{*}\right\rangle$ and $h \uparrow\left[u_{\varepsilon}\right]^{2} \equiv 1$ (for each $\varepsilon<\lambda$ ) then there are $\alpha<\beta$ (really a large set of these) such that

$$
h \uparrow\left[u_{\alpha} \cup u_{\beta}\right]^{2} \equiv 1 .
$$

The point is that we can deal with functions with infinitely many variables. Looking at previous proofs, "in stronger" we can get (for $\mu$ strong limit singular etc.): for $\alpha$ large enough, for $i<\delta=\operatorname{cf}(\mu)$ large enough, etc. we can defeat

$$
\left(\ldots\left(\forall^{I_{\eta_{\varepsilon, l}}{ }^{\uparrow i}} \gamma_{l}\right) \ldots\right)\left(\left\langle\gamma_{l}: l<l^{*}\right\rangle \in B_{\left\langle\eta_{\alpha_{\varepsilon}, l} \mid i: l<l^{*}\right\rangle}\right)
$$

but the duality of quantifiers fails, so the conclusion is only that

$$
\left(\forall^{J} i<\delta\right)\left[\neg\left(\ldots\left(\forall^{I_{\eta_{\varepsilon}, l}{ }^{i j}} \gamma_{l}\right) \ldots\right)_{l<l^{*}}\left(\left\langle\eta_{\alpha_{\varepsilon, l}}(i): l<l^{*}\right\rangle \notin B_{\left\langle\eta_{\alpha_{\varepsilon} l} \backslash i: l<l^{*}\right\rangle}\right)\right] .
$$

(6) (no ultrafilters) If $I \supseteq J_{\eta}^{\text {bd }}, \delta$ is a regular cardinal, $\lambda_{\eta}=\lambda_{\lg (\eta)}$ and for each $u \in\left[T_{i}\right]^{<|\delta| \chi}, i<\delta$ the free product $\star_{\eta \in u} \mathbb{B}_{\eta}$ satisfies the $\lambda$-cc then we can show that the algebra $\mathbb{B}_{<\chi}^{\text {red }}$ also satisfies the $\lambda$-cc, where for a cardinal $\chi$ the algebra $\mathbb{B}_{<\chi \chi}^{\text {red }}$ is the Boolean algebra freely generated by

$$
\begin{aligned}
\left\{\bigcap_{\alpha \in u} x_{\alpha}^{\mathfrak{t}(\alpha)}:\right. & \mathfrak{t}: u \rightarrow 2, u \in[\lambda]^{<\delta}, h \uparrow\left[u \cap \mathfrak{t}^{-1}[1]\right]^{2} \equiv 1,|u|<\chi \text { and } \\
& \left.\left.(\exists i<\delta) \text { (the mapping } \alpha \mapsto \eta_{\alpha}(i) \text { is one-to-one (for } \alpha \in u\right)\right), \\
& \left.(\exists i<\delta)(\exists \alpha \in u)(\forall j \in(i, \delta))(\forall \beta \in u)\left(f_{\alpha}(j) \leq f_{\beta}(j)\right)\right\} .
\end{aligned}
$$

[Note that if $\chi \leq \operatorname{cf}(\delta)$ it is simpler.]

Now we will deal with an additional demand that the algebra $\mathbb{B}^{\text {red }}$ satisfies the $|\delta|^{+}$-cc (or even has the $|\delta|^{+}$-Knaster property). Note that the demand of $|\delta|$-cc does not seem to be reasonable: if every $\bar{y}_{\eta}$ has two disjoint members (and every node $t \in T$ is an initial segment of a branch $\left\{\eta_{\alpha}: \alpha<\lambda\right\}$ through $T$ and $\delta \neq \operatorname{cf}(\delta)$ implies $t$ has at least two immediate successors) then we can find $\delta$ branches which give $\delta$ pairwise disjoint elements. Moreover, for each $\nu \in T_{i}$ let $A_{\nu}=\left\{\eta_{\alpha}(i): \eta_{\alpha}\lceil i=\nu\}\right.$ and

$$
a_{\alpha}=\left\{i<\delta:\left(\exists \beta \in A_{\eta_{\alpha} \backslash i}\right)\left(\mathbb{B}_{\eta_{\alpha} \uparrow i}=y_{\eta_{\alpha}(i)} \cap y_{\beta}=\mathbf{0}\right)\right\} .
$$

So if $\mathbb{B}^{\text {red }}=\sigma$-cc then $(\forall \alpha<\lambda)\left(\left|a_{\alpha}\right|<\sigma\right)$.

Definition 5.12. Let $(T, \bar{\lambda}) \in \mathcal{K}_{\mu, \delta}$ and let $\bar{\eta}=\left\langle\eta_{\alpha}: \alpha<\lambda\right\rangle \subseteq \lim _{\delta}(T)$. We say that $\bar{\eta}$ is hereditary ${ }^{\dagger} \theta$-free if for every $Y \in[\lambda]^{\theta}$ there are $Z \in[Y]^{\theta}$ and $i<\delta$ such that

$$
(\forall \alpha, \beta \in Z)\left(\alpha \neq \beta \Rightarrow\left[\eta_{\alpha}\left\lceil i=\eta_{\beta}\left\lceil i \& \eta_{\alpha}(i) \neq \eta_{\beta}(i)\right]\right) .\right.\right.
$$

\footnotetext{
$\dagger$ Sorry, this is weaker than $\theta$-free.
} 
Proposition 5.13. Assume that $\mathcal{C}=\left(T, \bar{\lambda}, \bar{\eta},\left\langle\left(\mathbb{B}_{\eta}, \bar{y}_{\eta}\right): \eta \in T\right\rangle\right)$ is $a(\delta, \mu, \lambda)$-constructor. If $\bar{\eta}$ is hereditary $\theta$-free, each algebra $\mathbb{B}_{\eta}$ has the $\theta$ Knaster property and $\theta$ is regular then the algebra $\mathbb{B}^{\text {red }}(\mathcal{C})$ has the $\theta$-Knaster property.

Proof. The same as for 3.8. Note that the proof there shows actually that if $(\forall \alpha<\theta)\left(|\alpha|^{|\delta|}<\theta=\operatorname{cf}(\theta)\right)$, then $\bar{\eta}$ is $\theta$-hereditary free. Also if $(\forall \alpha<\theta)\left(|\alpha|^{<|\delta|}<\theta=\operatorname{cf}(\theta)\right)$ then we can weaken the demand in 5.12 to $(\forall \alpha, \beta \in Z)\left(\alpha \neq \beta \Rightarrow \eta_{\alpha}\left\lceil i \neq \eta_{\beta}\lceil i)\right.\right.$; note that we can replace $i$ by $i+1$.

Proposition 5.14. Assume that $(T, \bar{\lambda}) \in \mathcal{K}_{\mu, \delta}, \bar{\eta}=\left\langle\eta_{\alpha}: \alpha<\lambda\right\rangle \subseteq$ $\lim _{\delta}(T)$ and $\lambda$ is a regular cardinal. Further suppose that: $J_{\delta}^{\mathrm{bd}}$

(a) $(\forall \alpha<\theta)\left(|\alpha|^{<\delta}<\theta=\operatorname{cf}(\theta)\right), \delta<\theta$, J is an ideal on $\delta$ extending

(b) the sequence $\bar{\eta}$ is $<_{J}$-increasing and one of the following conditions is satisfied:

( $\alpha) \bar{\eta}$ is $<_{J}$-cofinal in $\prod_{i<\delta} \lambda_{i} / J, \lambda_{i}$ are regular cardinals above $\theta$ (at least for J-majority of $i<\delta),\{\alpha<\lambda: \operatorname{cf}(\alpha)=\theta\} \in I[\lambda]$ and $\lambda_{\eta}=\lambda_{\lg (\eta)}$,

( $\beta$ ) there are a sequence $\left\langle C_{\alpha}: \alpha<\lambda\right\rangle$ of subsets of $\lambda$, a closed unbounded subset $E$ of $\lambda$ and $i^{*}<\delta$ such that:

(i) $C_{\alpha} \subseteq \alpha, \operatorname{otp}\left(C_{\alpha}\right) \leq \theta$,

(ii) if $\beta \in C_{\alpha}$ then $C_{\beta}=C_{\alpha} \cap \beta$ and $\eta_{\beta}\left\lceil\left[i^{*}, \delta\right)<\eta_{\alpha}\left\lceil\left\lceil i^{*}, \delta\right)\right.\right.$,

(iii) if $\alpha \in E$ and $\operatorname{cf}(\alpha)=\theta$ then $\alpha=\sup \left(C_{\alpha}\right)$.

Then there is $A \in[\lambda]^{\lambda}$ such that the restriction $\bar{\eta}\lceil A$ is $\theta$-hereditary free.

Proof. First assume that case $(b)(\beta)$ holds.

Claim 5.14.1. Suppose that $Y \in[E]^{\theta}$. Then:

(1) $\left(\exists Z \in[Y]^{\theta}\right)\left(\exists i^{\otimes}\right)\left(\left\langle f_{\beta_{\varepsilon}}\left(i^{\otimes}\right): \varepsilon \in Z\right\rangle\right.$ is strictly increasing $)$.

(2) If additionally $J=J_{\delta}^{\mathrm{bd}}$ then

$\left(\exists Z \in[Y]^{\theta}\right)\left(\exists i^{\otimes}<\delta\right)\left(\left\langle\eta_{\beta}\left\lceil\left[i^{\otimes}, \delta\right): \beta \in Z\right\rangle\right.\right.$ is strictly increasing $)$.

Proof. Suppose $Y \in[E]^{\theta}$. Without loss of generality we may assume that $\operatorname{otp}(Y)=\theta$. Let $\alpha=\sup (Y)$. So $\alpha \in E, \operatorname{cf}(\alpha)=\theta$ and hence $C_{\alpha}$ is unbounded in $\alpha$. Let $C_{\alpha}=\left\langle\alpha_{\varepsilon}: \varepsilon<\theta\right\rangle$ be the increasing enumeration. Clearly the set

$$
A:=\left\{\varepsilon<\theta:\left[\alpha_{\varepsilon}, \alpha_{\varepsilon+1}\right) \cap Y \neq \emptyset\right\}
$$

is unbounded in $\theta$. For $\varepsilon \in A$ choose $\beta_{\varepsilon} \in\left[\alpha_{\varepsilon}, \alpha_{\varepsilon+1}\right) \cap Y$. Then

$$
\left(\exists a_{\varepsilon} \in J\right)\left(\eta_{\alpha_{\varepsilon}}\left\lceil\left(\delta \backslash a_{\varepsilon}\right) \leq \eta_{\beta_{\varepsilon}} \uparrow\left(\delta \backslash a_{\varepsilon}\right)<\eta_{\alpha_{\varepsilon+1}} \uparrow\left(\delta \backslash a_{\varepsilon}\right)\right) .\right.
$$

Now choose $i_{\varepsilon} \in \delta \backslash a_{\varepsilon}, i_{\varepsilon}>i^{*}$ and find $B \in[A]^{\theta}$ such that

$$
\varepsilon \in B \quad \Rightarrow \quad i_{\varepsilon}=i^{\otimes} \text {. }
$$


Clearly, by the assumption $(\beta)\left(\right.$ ii), this $i^{\otimes}$ and $Z=\left\{\beta_{\varepsilon}: \varepsilon \in B\right\}$ are as required in 5.14.1(1).

If additionally we know that $J=J_{\delta}^{\mathrm{bd}}$ then for some $B \in[A]^{\theta}$ we have

$$
\left(\exists i^{\otimes} \in\left[i^{*}, \delta\right)\right)\left(\varepsilon \in B \Rightarrow a_{\varepsilon} \subseteq i^{\otimes}\right)
$$

and hence the sequence $\left\langle f_{\beta_{\varepsilon}} \uparrow\left[i^{\otimes}, \delta\right): \varepsilon \in B\right\rangle$ is as required in 5.14.1(2) (remember $(\beta)($ ii $))$.

But now, using $i^{\otimes}$ given by 5.14 .1 we may deal with the sequence $\left\langle f_{\beta_{\varepsilon}} \uparrow\left(i^{\otimes}+1\right): \varepsilon \in B\right\rangle$ and using the old proof (see 3.8) on the tree $\bigcup_{i \leq i \otimes} T_{i}$ (note that we may apply the assumption (a) to arguments like there) we may get the desired conclusion. This finishes the case when $(b)(\beta)$ holds true.

Now, assume that (b) ( $\alpha$ ) holds. We reduce this case to the previous one (using cofinality).

Take $\bar{C}, E$ witnessing that the set $\{\alpha<\lambda: \operatorname{cf}(\alpha)=\theta\}$ is in $I[\lambda]$ and build a $<_{J}$-increasing sequence $\bar{\eta}^{\prime}=\left\langle\eta_{\alpha}^{\prime}: \alpha<\lambda\right\rangle \subseteq \prod_{i<\delta} \lambda_{i}$ such that $\eta_{\alpha}^{\prime}>\eta_{\alpha}$ and $\bar{\eta}^{\prime}$ satisfies clause $(\beta)$ of (b) for $\bar{C}, E$. [The construction of $\eta_{\alpha}^{\prime}$ is by induction on $\alpha<\lambda$. Suppose that we have defined $\eta_{\beta}^{\prime}$ for $\beta<\alpha$. Now, at stage $\alpha$ of the construction, we first choose $\eta_{\alpha}^{0} \in \prod_{i<\delta} \lambda_{i}$ such that

$$
(\forall \beta<\alpha)\left(\eta_{\beta}^{\prime}<_{J} \eta_{\alpha}^{0}\right) .
$$

This is possible since the condition $(\alpha)$ implies that $\lambda=\operatorname{tcf}\left(\prod_{i<\delta} \lambda_{i} / J\right)$ and $\alpha<\lambda$. Now for $i<\delta$ we put

$$
\eta_{\alpha}^{\prime}(i)=\max \left\{\eta_{\alpha}^{0}(i), \eta_{\alpha}(i)+1, \sup \left\{\eta_{\gamma}^{\prime}(i)+1: \gamma \in C_{\alpha}\right\}\right\} .
$$

One can check that this $\bar{\eta}^{\prime}$ is as required.]

Now we use the fact that $\bar{\eta}$ is cofinal. The set

$$
E^{\prime}=\left\{\gamma \in E:(\forall \alpha<\gamma)(\exists \beta<\gamma)\left(\eta_{\alpha}^{\prime}<_{J} \eta_{\beta}\right)\right\}
$$

is a club of $\lambda$. Look at $\bar{\eta}\left\lceil E^{\prime}\right.$. Suppose that $Y \in\left[E^{\prime}\right]^{\theta}$. Without loss of generality we may assume that $\operatorname{otp}(Y)=\theta$ and let $\alpha=\sup (Y)$. By induction on $\varepsilon<\theta$ choose $\alpha_{\varepsilon}<\beta_{\varepsilon}<\gamma_{\varepsilon}$ such that $\beta_{\varepsilon} \in Y, \alpha_{\varepsilon} \in C_{\alpha}$, $\gamma_{\varepsilon} \in C_{\alpha}, \eta_{\alpha_{\varepsilon}}^{\prime}<_{J} \eta_{\beta_{\varepsilon}}<_{J} \eta_{\gamma_{\varepsilon}}^{\prime}$ and if $\zeta<\varepsilon$ then $\gamma_{\zeta}<\alpha_{\varepsilon}$. Next choose $i_{\varepsilon}>i^{*}$ such that

$$
\eta_{\alpha_{\varepsilon}}^{\prime}\left(i_{\varepsilon}\right)<\eta_{\beta_{\varepsilon}}\left(i_{\varepsilon}\right)<\eta_{\gamma_{\varepsilon}}^{\prime}\left(i_{\varepsilon}\right)
$$

We may assume that $i_{\varepsilon}=i^{\otimes}$ for all $\varepsilon<\theta$. Now, as $\bar{\eta}^{\prime}$ obeys $\bar{C}$, we have

$$
\begin{aligned}
\zeta<\varepsilon & \Rightarrow \eta_{\gamma_{\zeta}}^{\prime}\left(i^{\otimes}\right)<\eta_{\alpha_{\varepsilon}}^{\prime}\left(i^{\otimes}\right), \\
J=J_{\delta}^{\mathrm{bd}} \wedge \zeta<\varepsilon & \Rightarrow \quad \eta_{\gamma_{\zeta}}^{\prime} \uparrow\left[i^{\otimes}, \delta\right)<\eta_{\alpha_{\varepsilon}}^{\prime} \uparrow\left[i^{\otimes}, \delta\right),
\end{aligned}
$$

and hence we conclude that the sequence $\left\langle\eta_{\beta_{\varepsilon}}\left(i^{\otimes}\right): \varepsilon<\theta\right\rangle$ is strictly increasing. Now we may finish the proof as earlier. 
Conclusion 5.15. If $\mu$ is a strong limit singular cardinal, $2^{\mu}=\mu^{+}=\lambda$ and $\neg\left(\exists 0^{\#}\right)$ or at least

$$
\left\{\delta<\mu^{+}: \operatorname{cf}(\delta)=\left(2^{<\operatorname{cf}(\mu)}\right)^{+}\right\} \in I[\lambda]
$$

then there is a $(\operatorname{cf}(\mu), \mu, \lambda)$-constructor $\mathcal{C}$ such that the algebra $\mathbb{B}^{\mathrm{red}}(\mathcal{C})$ has the $\left(2^{<\mathrm{cf}(\mu)}\right)^{+}$-Knaster property, its counterpart $\mathbb{B}^{\text {green }}(\mathcal{C})$ is $\lambda$-cc and the free product is not $\lambda$-cc.

[Note that if GCH holds then $\left(2^{<\operatorname{cf}(\mu)}\right)^{+}=(\operatorname{cf}(\mu))^{+}$so the problem is closed then.]

Proof. Like 4.4 using 5.14, 5.13 instead of $2.8,3.8$.

6. The use of pcf. Assuming that $2^{<\kappa}$ is much larger than $\kappa=\operatorname{cf}(\kappa)$ $(=\operatorname{cf}(\mu)<\mu)$ we may still want to have examples with the $\left(\kappa^{+}, \operatorname{not} \lambda\right)$ Knaster property and the non-multiplicativity. Here 5.15 does not help if GCH holds on an end segment of the cardinals (and $\neg\left(\exists 0^{\#}\right)$ ). We try to remedy this.

It is done inductively. So 6.3 uses $\operatorname{cf}(\mu)=\aleph_{0}$ just to start the induction. We can phrase (a part of) it without this assumption but in applications we use it for $\operatorname{cf}(\mu)=\aleph_{0}$. Also 6.3(b) really needs this condition (otherwise we would have to assume that $\left.(\forall \alpha<\theta)\left(|\alpha|^{<\delta}<\mu\right)\right)$. This result says that, if $\operatorname{cf}(\mu)=\aleph_{0}$, then we have the $\theta$-Knaster property for every regular cardinal $\theta \in \mu \backslash \kappa^{+}$.

Definition 6.1. (1) Let $\mathcal{K}_{\text {wmk }}$ denote the class of all tuples $(\theta, \lambda, \chi, J)$ such that $\theta<\lambda, \chi$ are regular cardinals, $J$ is a $\chi$-complete ideal on $\lambda$ and there is a $(\lambda, \chi)$-well marked Boolean algebra $(\mathbb{B}, \bar{y}, J)$ (see 3.2) such that the algebra $\mathbb{B}$ has the $\theta$-Knaster property (wmk stands for "well marked Knaster").

When we write $(\theta, \lambda) \in \mathcal{K}_{\text {wmk }}$ we really mean $\left(\theta, \lambda, \lambda, J_{\lambda}^{\text {bd }}\right) \in \mathcal{K}_{\text {wmk }}$ (which just means that there exists a $(\theta, \lambda)$-Knaster marked Boolean algebra).

(2) By $\mathcal{K}_{\text {smk }}$ (smk is for "sequence marked Knaster") we denote the class of all triples $(\theta, \lambda, \chi)$ of cardinals such that $\theta<\lambda$ are regular and there is a sequence $\left\langle\left(\mathbb{B}_{\alpha}, \bar{y}^{\alpha}\right): \alpha<\chi\right\rangle$ of $\lambda$-marked Boolean algebras such that (for $\alpha<\chi)$ the algebras $\mathbb{B}_{\alpha}$ have the $\theta$-Knaster property, $\bar{y}^{\alpha}=\left\langle y_{i}^{\alpha}: i<\lambda\right\rangle$ and if $n<\omega, \alpha_{0}<\ldots<\alpha_{n-1}<\chi$ and $\beta_{\varepsilon, l}<\lambda$ for $\varepsilon<\lambda, l<n$ are such that $\left(\forall \varepsilon_{1}<\varepsilon_{2}<\lambda\right)(\forall l<n)\left(\beta_{\varepsilon_{1}, l}<\beta_{\varepsilon_{2}, l}\right)$ then there are $\varepsilon_{1}<\varepsilon_{2}<\lambda$ such that

$$
l<n \quad \Rightarrow \quad \mathbb{B}_{\alpha_{l}} \models \text { " } y_{\beta_{\varepsilon_{1}, l}}^{\alpha_{l}} \cap y_{\beta_{\varepsilon_{2}, l}}^{\alpha_{l}}=\mathbf{0} \text { ". }
$$

Remark 6.2. (1) On some closure properties of $\mathcal{K}_{\text {wmk }}^{\theta}:=\{\lambda:(\theta, \lambda) \in$ $\left.\mathcal{K}_{\text {wmk }}\right\}$ under pcf see 3.12: if $\lambda_{i} \in \mathcal{K}_{\text {wmk }}^{\theta}$ (for $\left.i<\delta\right), \lambda_{i}>\max \operatorname{pcf}\left\{\lambda_{j}: j<i\right\}$ and $\lambda \in \operatorname{pcf}\left\{\lambda_{i}: i<\delta\right\}$ and $(\forall \alpha<\theta)\left(|\alpha|^{|\delta|}<\theta\right)$ then $\lambda \in \mathcal{K}_{\mathrm{wmk}}^{\theta}$. 
(2) We can replace $\theta$ by a set $\Theta$ of such cardinals, with no real difference. And thus we may consider the class $\mathcal{K}_{\mathrm{wmk}}^{*}$ of all tuples $(\Theta, \lambda, \chi, J)$ such that there exists a $(\lambda, \chi)$-well marked Boolean algebra $(\mathbb{B}, \bar{y}, J)$ with

$(\forall \theta \in \Theta)(\mathbb{B}$ has the $\theta$-Knaster property).

(3) In 6.1(2), each $\left(\mathbb{B}_{\alpha}, \bar{y}^{\alpha}\right)$ is well marked.

Proposition 6.3. Assume that $\mu$ is a strong limit singular cardinal, $\aleph_{0}=\operatorname{cf}(\mu)<\mu$ and $\lambda=2^{\mu}=\mu^{+}$.

(a) If $(\forall \alpha<\theta)\left(|\alpha|^{\operatorname{cf}(\mu)}<\theta=\operatorname{cf}(\theta)<\lambda\right)$, then $(\theta, \lambda) \in \mathcal{K}_{\text {wmk }}$. Moreover $\left(\theta, \lambda, 2^{\lambda}\right) \in \mathcal{K}_{\mathrm{smk}}$.

(b) If $\operatorname{cf}(\mu)<\theta=\operatorname{cf}(\theta)<\mu$ and $\{\alpha<\lambda: \operatorname{cf}(\alpha)=\theta\} \in I[\lambda]$, then $(\theta, \lambda) \in \mathcal{K}_{\text {wmk }}$. Moreover $\left(\theta, \lambda, 2^{\lambda}\right) \in \mathcal{K}_{\text {smk }}$.

Proof. This is similar to previous proofs and the first parts of 6.3(a), (b) follow from what we have done already: (a) is an obvious modification of 3.11 ; (b) is similar, but based on 5.13, 5.14 (and 2.8, 3.7) (see below). What we actually have to prove are the "moreover" parts. We will only sketch the proof for (b), modifying the proof of 4.4 .

As in 4.4 we choose $h: \operatorname{cf}(\mu) \rightarrow \omega$ such that for each $n \in \omega$ the preimage $h^{-1}[\{n\}]$ is unbounded (in $\left.\operatorname{cf}(\mu)\right)$. Next we take an increasing sequence $\left\langle\mu_{i}: i<\operatorname{cf}(\mu)\right\rangle$ of regular cardinals such that $\mu=\sum_{i<\delta} \mu_{i}$. Finally (as in 4.4) we construct $\lambda_{i}, \chi_{i},\left(\mathbb{B}_{i}, \bar{y}_{i}\right)$ and $I_{i}$ such that for $i<\operatorname{cf}(\mu)$ :

(1) $\lambda_{i}, \chi_{i}<\mu$ are regular cardinals,

(2) $\lambda_{i}>\chi_{i} \geq \prod_{j<i} \lambda_{j}+\mu_{i}$ and $\chi_{0}>\theta+\mu_{0}$,

(3) $I_{i}$ is a $\chi_{i}^{+}$-complete ideal on $\lambda_{i}$,

(4) $\left(\mathbb{B}_{i}, \bar{y}_{i}\right)$ is a $\lambda_{i}$-marked Boolean algebra such that if $n=h(i)$ and the set $Y \subseteq\left(\lambda_{i}\right)^{n+1}$ is such that

$$
\left(\exists^{I_{i}} \gamma_{0}\right) \ldots\left(\exists^{I_{i}} \gamma_{n}\right)\left(\left\langle\gamma_{0}, \ldots, \gamma_{n}\right\rangle \in Y\right)
$$

then for some $\gamma_{l}^{\prime}, \gamma_{l}^{\prime \prime}<\lambda_{i}($ for $l \leq n)$ we have $\left\langle\gamma_{l}^{\prime}: l \leq n\right\rangle,\left\langle\gamma_{l}^{\prime \prime}: l \leq n\right\rangle \in Y$ and for all $l \leq n$,

$$
\mathbb{B}_{i}=y_{\gamma_{l}^{\prime}}^{i} \cap y_{\gamma_{l}^{\prime \prime}}^{i}=\mathbf{0},
$$

(5) each algebra $\mathbb{B}_{i}$ satisfies the $\theta$-Knaster condition,

(6) for $\xi<\lambda_{i}$ the set $\left[\xi, \lambda_{i}\right)$ is not in the ideal $I_{i}$.

Note that the last requirement is new here. Though we cannot demand that the ideals $I_{i}$ extend $I_{\lambda_{i}}^{\mathrm{bd}}$, the condition (6) above is satisfied in our standard construction. Note that the ideal from 3.1 has this property if $\lambda$ there is regular. Moreover it is preserved when the (finite) products of ideals (as in 4.2) are considered. Also, if $I$ is an ideal on $\lambda, A_{0} \in I$ is such that $\left|\lambda \backslash A_{0}\right|$ is minimal and $A_{1} \in I^{+}$is such that $\left|A_{1}\right|$ is minimal then we can use either 
$I\left\lceil A_{0}\right.$ or $I\left\lceil A_{1}\right.$. All relevant information is then preserved (in the first case the condition (6) holds, in the second $J_{\lambda}^{\text {bd }} \subseteq I$ under suitable renaming).

Now we put $T=\bigcup_{i<\delta} \prod_{j<i} \lambda_{j}, \mathbb{B}_{\eta}=\mathbb{B}_{\lg (\eta)}, \bar{y}_{\eta}=\bar{y}_{\lg (\eta)}$ and $I_{\eta}=I_{\lg (\eta)}$. Applying 2.8 we find a stronger $J_{\delta}^{\mathrm{bd}}$-cofinal sequence $\bar{\eta}=\left\langle\eta_{\alpha}: \alpha<\lambda\right\rangle$ for $(T, \bar{\lambda}, \bar{I})$. By (6) we may additionally demand that $\bar{\eta}$ is $<_{J_{\mathrm{cf}(\mu)}^{\mathrm{bd}}}$-increasing cofinal in $\prod_{i<\operatorname{cf}(\mu)} \lambda_{i} / J_{\operatorname{cf}(\mu)}^{\mathrm{bd}}$. Let $\left\langle B_{\xi}: \xi<2^{\lambda}\right\rangle$ be a sequence of pairwise almost disjoint elements of $[\lambda]^{\lambda}$ (i.e., $\left|B_{\xi} \cap B_{\zeta}\right|<\lambda$ for distinct $\xi, \zeta<2^{\lambda}$ ). For each $\xi<2^{\lambda}$ we may apply 5.14 (the version of $\left.(\mathrm{b})(\alpha)\right)$ to the sequence $\left\langle\eta_{\alpha}: \alpha \in B_{\xi}\right\rangle$ and we find $A_{\xi} \in\left[B_{\xi}\right]^{\lambda}$ such that each sequence $\left\langle\eta_{\alpha}: \alpha \in A_{\xi}\right\rangle$ is $\theta$-hereditary free. Let

$$
\mathbb{B}_{\xi}^{*}=\mathbb{B}^{\mathrm{red}}\left(T, \bar{\lambda},\left\langle\eta_{\alpha}: \alpha \in A_{\xi}\right\rangle,\left\langle\left(\mathbb{B}_{\eta}, \bar{y}_{\eta}\right): \eta \in T\right\rangle\right), \quad \bar{x}_{\xi}=\left\langle x_{\alpha}^{\mathrm{red}}: \alpha \in A_{\xi}\right\rangle .
$$

Of course, each $\mathbb{B}_{\xi}^{*}$ is a subalgebra of $\mathbb{B}^{\text {red }}\left(T, \bar{\lambda}, \bar{\eta},\left\langle\left(\mathbb{B}_{\eta}, \bar{y}_{\eta}\right): \eta \in T\right\rangle\right.$ ) (generated by $\bar{x}_{\xi}$ ). By 5.13 and 3.7 we know that the marked Boolean algebras $\left(\mathbb{B}_{\xi}^{*}, \bar{x}_{\xi}\right)$ are $(\theta$, not $\lambda)$-Knaster. To show that they witness $\left(\theta, \lambda, 2^{\lambda}\right) \in \mathcal{K}_{\text {smk }}$ suppose that $n<\omega, \xi_{0}, \ldots, \xi_{n-1}<2^{\lambda}$ and $\beta_{\varepsilon, l}<\lambda$ (for $\varepsilon<\lambda, l<n$ ) are such that

$$
\left(\forall \varepsilon_{1}<\varepsilon_{2}<\lambda\right)(\forall l<n)\left(\beta_{\varepsilon_{1}, l}<\beta_{\varepsilon_{2}, l}\right),
$$

and of course $\left\{\beta_{\varepsilon, l}: \varepsilon<\lambda\right\} \subseteq A_{\xi_{l}}$. Since $A_{\xi_{l}}$ are almost disjoint we may assume that

$$
\left(\forall \varepsilon_{1}, \varepsilon_{2}<\lambda\right)\left(\forall l_{1}<l_{2}<n\right)\left(\beta_{\varepsilon_{1}, l_{1}} \neq \beta_{\varepsilon_{2}, l_{2}}\right) .
$$

Further we may assume that we have $i^{*}<\operatorname{cf}(\mu)$ such that for each $\varepsilon<\lambda$ the sequences $\eta_{\beta_{\varepsilon, l}}\left\lceil i^{*}\right.$ for $l<n$ are pairwise distinct.

By the choice of $\bar{\eta}, T, \bar{\lambda}$ etc. we may apply 4.3 .1 to conclude that for all sufficiently large $\varepsilon<\lambda$ the set

$$
\begin{array}{r}
Z_{\varepsilon}=\left\{i<\operatorname{cf}(\mu): \neg\left(\exists^{I_{\eta_{\beta_{\varepsilon}, 0}}{ }^{i i}} \gamma_{0}\right) \ldots\left(\exists^{I_{\eta_{\beta_{\varepsilon, n-1}}}{ }^{i i}} \gamma_{n-1}\right)\left(\exists^{\lambda} \zeta\right)(\forall l<n)\right. \\
\left(\eta_{\beta_{\varepsilon, l}} \uparrow(i+1)=\left(\eta_{\beta_{\varepsilon, l}}\lceil i) \frown\left\langle\gamma_{l}\right\rangle\right)\right\}
\end{array}
$$

is in the ideal $J_{\operatorname{cf}(\mu)}^{\mathrm{bd}}$. Take one such $\varepsilon$. Choosing $i \in \operatorname{cf}(\mu) \backslash Z_{\varepsilon}, i>i^{*}$ such that $h(i)=n$ we may follow exactly the last part of the proof of 4.3 to find $\varepsilon_{0}, \varepsilon_{1}<\lambda$ such that for each $l<n$,

$$
\eta_{\beta_{\varepsilon_{0}, l}}\left\lceil i=\eta_{\beta_{\varepsilon_{1}, l}} \uparrow i, \quad \text { but } \quad \mathbb{B}_{\eta_{\beta_{\varepsilon_{0}, l}} \uparrow i}=y_{\eta_{\beta_{\varepsilon_{0}, l}} \uparrow(i+1)} \cap y_{\eta_{\beta_{\varepsilon_{1}, l}} \uparrow(i+1)}=\mathbf{0},\right.
$$

which implies that

$$
(\forall l<n)\left(\mathbb{B}_{\xi_{l}}^{*} \models x_{\beta_{\varepsilon_{0}, l}}^{\mathrm{red}} \cap x_{\beta_{\varepsilon_{1}, l}}^{\mathrm{red}}=\mathbf{0}\right) .
$$

Proposition 6.4. Assume that:

(a) $\left\langle\lambda_{i}: i<\delta\right\rangle$ is an increasing sequence of regular cardinals such that $\delta<\lambda_{0}$ and $\lambda_{i}>\max \operatorname{pcf}\left\{\lambda_{j}: j<i\right\}$ (the last is our natural assumption),

(b) $\aleph_{0}<\theta=\operatorname{cf}(\theta)<\bigcup_{i<\delta} \lambda_{i}$ (naturally we assume just $\operatorname{cf}(\theta)=\theta<\lambda_{0}$ ), 
(c) $\lambda=\max \operatorname{pcf}\left\{\lambda_{i}: i<\delta\right\}$,

(d) $\left(\theta, \lambda_{i}, \max \operatorname{pcf}\left\{\lambda_{j}: j<i\right\}\right) \in \mathcal{K}_{\mathrm{smk}}$,

(e) for each $\tau \in\{\lambda\} \cup \bigcup_{\alpha<\delta} \operatorname{pcf}\left\{\lambda_{i}: i<\alpha\right\}$ we have

$$
\{\xi<\tau: \operatorname{cf}(\xi)=\theta\} \in I[\tau],
$$

or at least for some $\bar{f}^{\tau}=\left\langle f_{\varepsilon}^{\tau}: \varepsilon<\tau\right\rangle,<_{J_{=\tau}}$-increasing cofinal in $\prod_{i<\alpha} \lambda_{i} / J_{=\tau}$ we have

$$
\gamma<\tau \& \operatorname{cf}(\gamma)=\theta \quad \Rightarrow \quad f_{\gamma}^{\tau} \text { is good in } \bar{f}^{\tau}
$$

(see [21], [20, §1 and 1.6(1)], and then Magidor and Shelah [9]),

(f) $\left|\operatorname{pcf}\left\{\lambda_{i}: i<\delta\right\}\right|<\theta$ or at least $\left|\operatorname{pcf}\left\{\lambda_{i}: i<\alpha\right\}\right|+|\delta|<\theta$ for each $\alpha<\delta$.

Then $(\theta, \lambda) \in \mathcal{K}_{\text {wmk }}$. Moreover $(\theta, \lambda, \chi) \in \mathcal{K}_{\text {smk }}$ provided there is an almost disjoint family of size $\chi$ in $[\lambda]^{\lambda}$. We may get algebras $\mathbb{B}^{\text {red }}, \mathbb{B}^{\text {green }}$ as in the main constructions such that

$$
\mathbb{B}^{\text {red }}=\theta \text {-Knaster }, \quad \mathbb{B}^{\text {green }}=\lambda \text {-cc, } \quad \mathbb{B}^{\text {red }} * \mathbb{B}^{\text {green }}=\neg \lambda \text {-cc. }
$$

REMark 6.4.A. This continues also the proof of $[22,3.5]$. Of course instead of clauses (e) $+(\mathrm{f})$ we may demand $(\forall \alpha<\theta)\left(|\alpha|^{|\delta|}<\theta=\operatorname{cf}(\theta)\right)$.

Proof. The main difficulty of the proof will be to construct a hereditary $\theta$-free $<_{J_{<\lambda}}$-increasing sequence $\bar{\eta}=\left\langle\eta_{\alpha}: \alpha<\lambda\right\rangle \subseteq \prod_{i<\delta} \lambda_{i}$. This is done in the claim below. For the notation used there let us note that if $\alpha \leq \delta$ is a limit ordinal, $\tau \in \operatorname{pcf}\left\{\lambda_{i}: i<\alpha\right\}$ then $J_{=\tau}\left[\left\{\lambda_{i}: i<\alpha\right\}\right]=J_{\tau}^{\alpha}$ is the ideal on $\alpha$ generated by

$$
J_{<\tau}\left[\left\{\lambda_{i}: i<\alpha\right\}\right] \cup\left\{\alpha \backslash \mathfrak{b}_{\tau}\left[\left\{\lambda_{i}: i<\alpha\right\}\right]\right\} .
$$

So in particular $\operatorname{tcf}\left(\prod_{i<\alpha} \lambda_{i} / J_{\tau}^{\alpha}\right)=\tau$.

Claim 6.4.1. There exists a tree $T \subseteq \bigcup_{i<\delta} \prod_{j<i} \lambda_{j}$ such that some $T_{\delta}^{\prime} \subseteq$ $\lim _{\delta}(T)$ is $\theta$-hereditary free (and $<_{J_{<\lambda}}$-cofinal). Moreover for each $\alpha<\delta$ the size of $T_{\alpha}$ is $\leq \max \operatorname{pcf}\left\{\lambda_{i}: i<\alpha\right\}$.

Proof. For a limit ordinal $\alpha \leq \delta$ and $\tau \in \operatorname{pcf}\left\{\lambda_{i}: i \leq \alpha\right\}$ (if $\alpha=\delta$ then

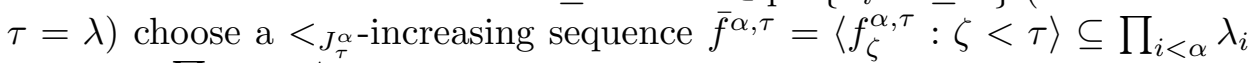
cofinal in $\prod_{i<\alpha} \lambda_{i} / J_{\tau}^{\alpha}$ and such that

$(\tilde{\otimes}) \quad$ if $\zeta<\tau, \operatorname{cf}(\zeta)=\theta$, then for some unbounded set $Y_{\zeta} \subseteq \zeta$ (for simplicity consisting of successor ordinals) and a sequence $\bar{s}^{\tau}=\left\langle s_{\xi}^{\tau}: \xi \in\right.$ $\left.Y_{\zeta}\right\rangle \subseteq J_{\tau}^{\alpha}$ we have

$$
\left[\xi_{1}, \xi_{2} \in Y_{\zeta} \& \xi_{1}<\xi_{2} \& i \in \alpha \backslash\left(s_{\xi_{1}}^{\tau} \cup s_{\xi_{2}}^{\tau}\right)\right] \quad \Rightarrow \quad f_{\xi_{1}}^{\alpha, \tau}(i)<f_{\xi_{2}}^{\alpha, \tau}(i) .
$$

[Why can we demand $(\tilde{\otimes})$ ? If in the assumption (e) the first part is satisfied then we argue similarly to the proof of 5.14 , compare $[20,1.5 \mathrm{~A}, 1.6$, pp. 
51-52]. If we are in the "at least" case then this is exactly the meaning of goodness.] Further we may demand that the sequence $\bar{f}^{\alpha, \tau}$ is ${ }^{b}$ continuous:

$(\tilde{\oplus}) \quad$ if $|\delta|<\operatorname{cf}(\zeta)<\lambda_{0}, \zeta<\tau$, then

$$
f_{\zeta}^{\alpha, \tau}(i)=\min \left\{\bigcup_{\xi \in C} f_{\xi}^{\alpha, \tau}(i): C \text { is a club of } \zeta\right\}
$$

[compare the proof of $[21,3.4$, pp. 25-26]].

For a limit ordinal $\alpha \leq \delta$ we define

$$
\begin{aligned}
T_{\alpha}^{0}=\left\{f \in \prod_{i<\alpha} \lambda_{i}:(\text { a) }\right. & f=\max \left\{f_{\zeta_{l}, \tau_{l}}^{\alpha, l} l<n\right\} \text { for some } n<\omega, \\
& \tau_{l} \in \operatorname{pcf}\left\{\lambda_{i}: i<\alpha\right\}, \text { and } \zeta_{l}<\tau_{l}, \\
\text { (b) } \text { for every } \tau \in \operatorname{pcf}\left\{\lambda_{i}: i<\alpha\right\}, & \\
& \text { if } \tau=\lambda \text { or } \alpha<\delta \text { then } \\
& \text { there is } \zeta_{f}(\tau)<\tau \text { such that } \\
& \left.f_{\zeta_{f}(\tau)}^{\alpha, \tau} \leq f \& f_{\zeta_{f}(\tau)}^{\alpha, \tau}=f \bmod J_{\tau}^{\alpha}\right\} .
\end{aligned}
$$

(Note that if $\alpha=\delta$ then there is only one value of $\tau_{l}, \tau$ which we consider here: $\lambda$.) Let $T^{\prime} \subseteq \bigcup_{i \leq \delta} \prod_{j<i} \lambda_{j}$ be the tree such that for $\gamma \leq \delta$,

$$
T_{\gamma}^{\prime}=\left\{f \in \prod_{i<\gamma} \lambda_{i}: f\left\lceil\alpha \in T_{\alpha}^{0} \text { for each limit } \alpha \leq \gamma\right\} .\right.
$$

Let

$$
\begin{aligned}
& A=\left\{\zeta<\lambda: \text { there is } f \in \prod_{i<\delta} \lambda_{i}\right. \text { such that } \\
& \left.\qquad f_{\zeta}^{\delta, \lambda} \leq f \& f_{\zeta}^{\delta, \lambda}=f \bmod J_{\lambda}^{\delta} \&(\forall i \leq \delta)\left(f\left\lceil i \in T_{i}^{\prime}\right)\right]\right\},
\end{aligned}
$$

and for each $\zeta \in A$ let $f_{\zeta}^{*}$ be a function witnessing it. Now, let $T \subseteq$ $\bigcup_{i<\delta} \prod_{j<i} \lambda_{j}$ be a tree such that $T_{\alpha}=\left\{f_{\zeta}^{*}\lceil\alpha: \zeta \in A\}\right.$.

By definition, $T$ is a tree, but maybe it does not have enough levels? Let $\chi$ be a large enough regular cardinal. Take an increasing continuous sequence $\left\langle N_{i}: i \leq \theta\right\rangle$ of elementary submodels of $\left(\mathcal{H}(\chi), \in,<^{*}\right)$ such that

$$
\left|N_{i}\right|=\Upsilon=\theta+\left|\operatorname{pcf}\left\{\lambda_{\alpha}: \alpha<\delta\right\}\right|<\lambda_{0}, \quad \Upsilon+1 \subseteq N_{i} \in N_{i+1},
$$

and all relevant things are in $N_{0}$. We define $f^{*} \in \prod_{\alpha<\delta} \lambda_{\alpha}$ by

$$
f^{*}(\alpha)=\sup \left(\bigcup_{i<\theta} N_{i} \cap \lambda_{\alpha}\right) .
$$

As in [18, pp. 63-65], one proves that $f^{*}\left\lceil\alpha \in T_{\alpha}^{0}\right.$ for each limit $\alpha \leq \delta$. Hence for some $\zeta$ we have $f^{*}=f_{\zeta}^{\delta, \lambda} \bmod J_{\lambda}^{\delta}$ and $f_{\zeta}^{\delta, \lambda} \leq f^{*}$ thus $\zeta \in A$. Consequently, $A$ is unbounded in $\lambda$.

By induction on $\alpha \leq \delta$ we prove that

(ฮ) if $f_{\zeta} \in T_{\alpha}$ (for $\zeta<\theta$ ) are pairwise distinct, then there are $Z \in[\theta]^{\theta}$ and $j<\alpha$ such that

$$
\left(\forall \zeta_{0}, \zeta_{1} \in Z\right)\left(\zeta_{0} \neq \zeta_{1} \Rightarrow\left[f_{\zeta_{0}}\left\lceil j=f_{\zeta_{1}}\left\lceil j \& f_{\zeta_{0}}(j) \neq f_{\zeta_{1}}(j)\right]\right) .\right.\right.
$$


If $\alpha$ is a non-limit ordinal then this is trivial. So suppose that $\alpha$ is limit, $\alpha<\delta$. Then for some $\tau_{\zeta, l} \in \operatorname{pcf}\left\{\lambda_{i}: i<\alpha\right\}, \xi_{\zeta, l}<\tau_{\zeta, l}, n_{\zeta}<\omega$ (for $\zeta<\theta$, $\left.l<n_{\zeta}\right)$ we have

$$
f_{\zeta}=\max \left\{f_{\xi_{\zeta, l}}^{\alpha, \tau_{\zeta, l}}: l<n_{\zeta}\right\}
$$

As $\theta>\left|\operatorname{pcf}\left\{\lambda_{\beta}: \beta<\alpha\right\}\right|$ we may assume that $n_{\zeta}=n^{*}, \tau_{\zeta, l}=\tau_{l}$ and for each $l<n^{*}$ the sequence $\left\langle\xi_{\zeta, l}: \zeta<\theta\right\rangle$ is either constant or strictly increasing. Now, the second case has to occur for some $l$ and we may argue similarly to 5.14.1 and then apply the inductive hypothesis. We are left with the case $\alpha=\delta$. So let $f_{\zeta}=f_{\beta_{\zeta}}^{*}$ for $\zeta<\delta$ and continue as before (with $\lambda$ for $\tau_{l}$ ).

This ends the proof of the claim (note that the arguments showing that all the $T_{\alpha}^{0}$ are not empty prove actually that the tree $T$ has enough branches to satisfy our additional requirements).

Now let $T$ be a tree as in the claim above. Let $\bar{\eta}=\left\langle\eta_{\alpha}: \alpha<\lambda\right\rangle \subseteq \lim _{\delta}(T)$ be the enumeration of $\left\{f_{\zeta}^{*}: \zeta \in A\right\}$ from the proof such that $\bar{\eta}$ is $<_{J_{<\lambda}}$ increasing cofinal in $\prod_{i<\delta} \lambda_{i} / J_{<\lambda}$. By the assumption (d) for each $\eta \in T$ we find a marked Boolean algebra $\left(\mathbb{B}_{\eta}, \bar{y}_{\eta}\right)$ such that for every $i<\delta$ the sequence $\left\langle\left(\mathbb{B}_{\eta}, \bar{y}_{\eta}\right): \eta \in T_{i}\right\rangle$ witnesses that $\left(\theta, \lambda_{i},\left|T_{i}\right|\right) \in \mathcal{K}_{\text {smk }}$. These parameters determine a $(\delta, \mu, \lambda)$-constructor $\mathcal{C}$, so we have the corresponding Boolean algebra $\mathbb{B}^{\text {red }}(\mathcal{C})$ (and its counterpart $\mathbb{B}^{\text {green }}(\mathcal{C})$ ). To show that they have the required properties we follow exactly the proof that $(\theta, \lambda, \chi) \in \mathcal{K}_{\text {smk }}$, so we will present this proof only.

First note that by 5.13 the algebra $\mathbb{B}^{\text {red }}(\mathcal{C})$ has the $\theta$-Knaster property. Now, let $\left\langle A_{\zeta}: \zeta<\chi\right\rangle \subseteq[\lambda]^{\lambda}$ be such that

$$
\zeta_{1}<\zeta_{2}<\chi \Rightarrow\left|A_{\zeta_{1}} \cap A_{\zeta_{2}}\right|<\lambda
$$

Let $\bar{x}_{\zeta}=\left\langle x_{\xi}^{\text {red }}: \xi \in A_{\zeta}\right\rangle$ and let $\mathbb{B}_{\zeta}$ be the subalgebra of $\mathbb{B}^{\text {red }}(\mathcal{C})$ generated by $\bar{x}_{\zeta}$. We want to show that the sequence $\left\langle\left(\mathbb{B}_{\zeta}, \bar{x}_{\zeta}\right): \zeta<\chi\right\rangle$ witnesses $(\theta, \lambda, \chi) \in \mathcal{K}_{\mathrm{smk}}$. For this suppose that $\zeta_{0}<\ldots<\zeta_{n-1}<\chi, n<\omega$ and $\beta_{\varepsilon, l} \in A_{\zeta, l}$ are increasing with $\varepsilon$ (for $\varepsilon<\lambda, l<n$ ) and without loss of generality with no repetition. We may assume that

$$
(\forall l<n)(\forall \varepsilon<\lambda)\left(\beta_{\varepsilon, l} \notin \bigcup_{m \neq l} A_{\zeta_{m}}\right) .
$$

Further we may assume that for some $i^{*}<\delta$ and pairwise distinct $\eta_{l} \in T_{i^{*}}$ (for $l<n$ ) we have

$$
(\forall \varepsilon<\lambda)(\forall l<n)\left(\eta_{\beta_{\varepsilon, l}}\left\lceil i^{*}=\eta_{l}\right) .\right.
$$

Now we take $i \in\left[i^{*}, \delta\right)$ such that

$$
\left(\forall \gamma<\lambda_{i}\right)\left(\exists^{\lambda} \varepsilon<\lambda\right)(\forall l<n)\left(\eta_{\beta_{\varepsilon, l}}(i)>\gamma\right)
$$

(remember that each $\left\langle\eta_{\beta_{\varepsilon, l}}: \varepsilon<\lambda\right\rangle$ is $<_{J_{<\lambda}}$-cofinal). Since $\left|T_{i}\right|<\lambda_{i}$ we can 
find $\nu_{0}, \ldots, \nu_{n-1} \in T_{i}$ such that $\eta_{l} \unlhd \nu_{l}$ and

$$
\left(\forall \gamma<\lambda_{i}\right)\left(\exists^{\lambda} \varepsilon<\lambda\right)(\forall l<n)\left(\eta_{\beta_{\varepsilon, l}} l i=\nu_{l} \& \eta_{\beta_{\varepsilon, l}}(i)>\gamma\right) .
$$

Consequently, we may choose a sequence $\left\langle\left\langle\gamma_{\xi, l}: l<n\right\rangle: \xi\left\langle\lambda_{i}\right\rangle \subseteq \lambda_{i}\right.$ such that $\xi<\gamma_{\xi, l}$ and

$$
\left(\forall \xi<\lambda_{i}\right)\left(\exists^{\lambda} \varepsilon<\lambda\right)(\forall l<n)\left(\eta_{\beta_{\varepsilon, l}}\left\lceil(i+1)=\nu_{l} \frown\left\langle\gamma_{\xi, l}\right\rangle\right) .\right.
$$

Now we use the choice of $\left(\mathbb{B}_{\nu_{l}}, \bar{y}_{\nu_{l}}\right)$ (witnessing $\left.\left(\theta, \lambda_{i},\left|T_{i}\right|\right) \in \mathcal{K}_{\text {smk }}\right)$ and we find $\xi_{1}<\xi_{2}<\lambda_{i}$ such that

$$
(\forall l<n)\left(\mathbb{B}_{\nu_{l}} \models y_{\gamma_{\xi_{1}, l}}^{\nu_{l}} \cap y_{\gamma_{\xi_{2}, l}}^{\nu_{l}}=\mathbf{0}\right),
$$

which allows us to find $\varepsilon_{1}<\varepsilon_{2}<\lambda$ such that for each $l<n$ the intersection $x_{\beta_{\varepsilon_{1}, l}} \cap x_{\beta_{\varepsilon_{2}, l}}$ is $\mathbf{0}$.

CONCLUSION 6.5. If $\left\langle\mu_{i}: i \leq \kappa\right\rangle$ is a strictly increasing continuous sequence of strong limit singular cardinals such that $\kappa<\mu_{0}, 2^{\mu_{i}}=\mu_{i}^{+}$, $\kappa<\theta=\operatorname{cf}(\theta)<\mu_{0}$ and $(\forall \alpha<\theta)\left(|\alpha|^{\kappa}<\theta\right)$ or

$$
i \leq \kappa \Rightarrow\left\{\alpha<\mu_{i}^{+}: \operatorname{cf}(\alpha)=\theta\right\} \in I\left[\mu_{i}^{+}\right]
$$

then $\left(\theta, \mu_{\kappa}^{+}\right) \in \mathcal{K}_{\mathrm{wmk}}$ and we may construct the corresponding Boolean algebras $\mathbb{B}^{\text {red }}, \mathbb{B}^{\text {green }}$.

Proposition 6.6. Suppose that we have Boolean algebras $\mathbb{B}^{\text {red }}, \mathbb{B}^{\text {green }}$ such that

- $\mathbb{B}^{\text {red }}$ satisfies the $\theta$-Knaster condition,

- for each $n<\omega$ the free product $\left(\mathbb{B}^{\text {green }}\right)^{n}$ satisfies the $\lambda$-cc,

- the free product $\mathbb{B}^{\text {red }} * \mathbb{B}^{\text {green }}$ fails the $\lambda$-cc.

Then $(\theta, \lambda, \chi) \in \mathcal{K}_{\mathrm{smk}}$, where $\chi=\lambda^{+}$(or even if $\chi$ is such that there is an almost disjoint family $\mathcal{A} \subseteq[\lambda]^{\lambda}$ of size $\left.\chi\right)$.

Proof. We have $y_{\alpha} \in\left(\mathbb{B}^{\text {red }}\right)^{+}$and $z_{\alpha} \in\left(\mathbb{B}^{\text {green }}\right)^{+}$for $\alpha<\lambda$ such that if $\alpha<\beta<\lambda$ then

$$
\text { either } \quad \mathbb{B}^{\text {red }}=y_{\alpha} \cap y_{\beta}=\mathbf{0} \quad \text { or } \quad \mathbb{B}^{\text {green }} \models z_{\alpha} \cap z_{\beta}=\mathbf{0} .
$$

Let $A_{\zeta} \in[\lambda]^{\lambda}$ (for $\zeta<\chi$ ) be pairwise almost disjoint sets. We want to show that the sequence

$$
\left\langle\left(\mathbb{B}^{\mathrm{red}}, \bar{y} \uparrow A_{\zeta}\right): \zeta<\chi\right\rangle
$$

is a witness for $(\theta, \lambda, \chi) \in \mathcal{K}_{\text {smk }}$. So we are given $\zeta_{0}<\zeta_{1}<\ldots<\zeta_{n-1}<\chi$ and sequences $\left\langle\alpha_{\varepsilon, l}: \varepsilon\langle\lambda\rangle \subseteq A_{\zeta_{l}}\right.$ with no repetitions. Then for some $\varepsilon^{*}<\lambda$ we have

$$
\varepsilon^{*} \leq \varepsilon<\lambda \quad \Rightarrow \quad \alpha_{\varepsilon, l} \notin \bigcup_{m \neq l} A_{\zeta, m} .
$$

We should find $\varepsilon_{1}<\varepsilon_{2}$ such that for all $l<n$,

$$
\mathbb{B}^{\text {red }} \models y_{\alpha_{\varepsilon_{1}, l}} \cap y_{\alpha_{\varepsilon_{2}, l}}=\mathbf{0} .
$$


For this it is enough to find $\varepsilon^{*}<\varepsilon_{1}<\varepsilon_{2}$ such that for $l<n$,

$$
\mathbb{B}^{\text {green }}=z_{\alpha_{\varepsilon_{1}, l}} \cap z_{\alpha_{\varepsilon_{2}, l}} \neq \mathbf{0} .
$$

But this we easily get from the fact that the free product $\left(\mathbb{B}^{\text {green }}\right)^{n}$ satisfies the $\lambda$-cc.

Comment 6.7. (1) The proofs that the algebra $\mathbb{B}^{\text {green }}$ satisfies the $\lambda$-cc (see $4.3,6.4$ ) give that actually for each $n<\omega$ the product $\left(\mathbb{B}^{\text {green }}\right)^{n}$ satisfies the $\lambda$-cc. So it is reasonable to add it (though not needed originally).

(2) The " $\bar{\eta}$ is (strongly) $J$-cofinal for $(T, \bar{\lambda}, \bar{I})$ " has easy consequences for the existence of colourings.

REMARK 6.8. For $\mu$ strong limit singular we may sometimes get a cofinal sequence of length $\lambda \in\left(\mu, 2^{\mu}\right]$ without $2^{\mu}=\mu^{+}$. By $[23, \S 5]$, if:

(a) $I_{i}$ is a $\chi_{i}$-complete ideal, $\left|I_{i}\right|=\tau_{i}, \chi_{i}$ regular,

(b) $\chi_{i} \leq \tau_{i} \leq\left(\chi_{i}\right)^{+n^{*}}, n^{*}<\omega$

(c) $\operatorname{tcf}\left(\prod_{i<\delta}\left(\chi_{i}\right)^{+l} / J\right)=\lambda$ for each $l \leq n^{*}$,

then:

$(\alpha)$ there is a cofinal sequence in $\prod_{i<\delta}\left(\mathcal{P}\left(\lambda_{i}\right) / I_{i}\right) / J$, because

$(\beta)$ it has the true cofinality.

So if for arbitrarily large $\chi, 2^{\chi}=\chi^{+}, 2^{\chi^{+}}=\chi^{++}$then we have the ideal we want and maybe the pcf condition holds. Thus, combining this and 6.9 below, we find that there may be an example of our kind not because of $\mathrm{GCH}$ reasons, but still requiring some cardinal arithmetic assumptions.

Proposition 6.9. Suppose that $\left\langle\lambda_{i}: i<\delta\right\rangle$ is a strictly increasing sequence of regular cardinals, $I_{i}$ is a $\left(\prod_{j<i} \lambda_{j}\right)^{+}$-complete ideal on $\lambda_{i}$ (so $\left.\prod_{j<i} \lambda_{j}<\lambda_{i}\right)$ and $\left(\mathbb{B}_{i}, \bar{y}_{i}, I_{i}\right)$ is a $\lambda_{i}$-well marked Boolean algebra $($ for $i<\delta)$.

(1) Assume that $\prod_{i<\delta}\left(I_{i}, \subseteq\right) / J$ has true cofinality $\lambda$. Then there exists $a(\theta, \operatorname{not} \lambda)$-Knaster marked Boolean algebra.

(2) Suppose in addition that $h: \delta \rightarrow \omega$ is a function such that

$$
(\forall n<\omega)\left(h^{-1}[\{n\}] \in J^{+}\right)
$$

and $I_{i}^{[h(i)]}($ for $i<\delta)$ are the product ideals on $\left(\lambda_{i}\right)^{n}$ :

$$
I_{i}^{[h(i)]}:=\left\{B \subseteq\left(\lambda_{i}\right)^{n}: \neg\left(\exists^{I_{i}} \gamma_{0}\right) \ldots\left(\exists^{I_{i}} \gamma_{h(i)-1}\right)\left(\left\langle\gamma_{l}: l<h(i)\right\rangle \in B\right)\right\} .
$$

Assume that

$$
\lambda=\operatorname{tcf}\left(\prod_{i<\delta}\left(I_{i}^{[h(i)]}, \subseteq\right) / J\right)
$$

and that the $\left(\mathbb{B}_{i}, \bar{y}_{i}, I_{i}\right)$ satisfy the following requirement: 
$(\tilde{*})_{h(i)} \quad$ if $B \subseteq\left(\operatorname{dom}\left(\bar{y}_{i}\right)\right)^{h(i)}$ is such that

$$
\left(\exists^{I_{i}} \gamma_{0}\right) \ldots\left(\exists^{I_{i}} \gamma_{h(i)}\right)\left(\left\langle\gamma_{l}: l \leq h(i)\right\rangle \in B\right),
$$

then there are $\gamma_{l}^{\prime}, \gamma_{l}^{\prime \prime}<\lambda_{i}($ for $l \leq h(i))$ such that for each $l$,

$$
\mathbb{B}_{i}=y_{i, \gamma_{l}^{\prime}} \cap y_{i, \gamma_{l}^{\prime \prime}}=\mathbf{0} \text {. }
$$

Then we can conclude that $\left(\left(2^{|\delta|}\right)^{+}, \lambda, \lambda^{+}\right) \in \mathcal{K}_{\mathrm{smk}}$ and we have a pair of algebras $\left(\mathbb{B}^{\text {red }}, \mathbb{B}^{\text {green }}\right)$ as in main theorem 4.4 .

Proof. The main point here is that with our assumptions in hand we may construct a sequence $\left\langle\eta_{\alpha}: \alpha<\lambda\right\rangle \subseteq \prod_{i<\delta} \lambda_{i}$ which is quite stronger $J$-cofinal: it satisfies the requirement of $2.2(6)(\mathrm{b})$ weakened to the demand that the set there is not in the dual filter $J^{\mathrm{c}}$. Of course this is still enough to carry out our proofs and we may use such a sequence to build the right examples.

(1) Let $\left\langle\left\langle A_{i}^{\alpha}: i<\delta\right\rangle: \alpha<\lambda\right\rangle$ witness the true cofinality. By induction on $\alpha<\lambda$ choose $\gamma_{\alpha}<\lambda$ and $\eta_{\alpha} \in \prod_{i<\alpha} \lambda_{i}$ such that

- $\left\langle\left\{\eta_{\beta}(i)\right\}: i<\delta\right\rangle \in \prod_{i<\delta} I_{i}$,

- if $\beta<\alpha$ then $\gamma_{\beta}<\gamma_{\alpha}$ and $\left(\forall^{J} i\right)\left(\eta_{\beta}(i) \in A_{i}^{\gamma_{\alpha}}\right)$, and

- $\eta_{\alpha}(i) \notin A_{i}^{\gamma_{\alpha}}$.

For $\alpha=0$ or $\alpha$ limit, first choose $\gamma_{\alpha}=\sup \left\{\gamma_{\alpha_{1}}+1: \alpha_{1}<\alpha\right\}$ and then choose $\eta_{\alpha}(i)$ by induction on $i$.

For $\alpha=\alpha_{1}+1$ first note that

$$
\left\langle\left\{\eta_{\alpha_{1}}(i)\right\}: i<\delta\right\rangle \in \prod_{i<\delta} I_{i} .
$$

Hence for some $\gamma_{\alpha}^{0}<\lambda$ we have

$$
\left(\forall^{J} i\right)\left(\eta_{\alpha_{1}}(i) \in A_{i}^{\gamma_{\alpha}}\right)
$$

Let $\gamma_{\alpha}=\max \left\{\gamma_{\alpha_{1}}, \gamma_{\alpha}^{0}\right\}$. Now choose $\eta_{\alpha}(i)$ by induction on $i$.

As $I_{i}$ is $\left|T_{i}\right|^{+}$-complete, clearly $\left\langle\eta_{\alpha}: \alpha<\lambda\right\rangle$ is $J$-cofinal for $(T, J, \bar{I})$ and $3.7,3.8$ give the conclusion.

(2) The construction of $\bar{\eta}$ is in a sense similar to the one in the proof of 2.8 , but we use our cofinality assumptions. We have a cofinal sequence in $\prod_{i<\delta}\left(I_{i}^{[h(i)]}, \subseteq\right) / J$ :

$$
\left\langle\left\langle A_{i}^{\alpha}: i<\delta\right\rangle: \alpha<\lambda\right\rangle \text {. }
$$

For each $A_{i}^{\alpha}$ we have "Skolem functions" $f_{i, l}^{\alpha}$ for $l<h(i)$ (as in the proofs of $4.3 .1,5.4)$.

We define $\eta_{\alpha}$ by induction on $\alpha<\lambda$. In the exclusion list we put all substitutions by $\eta_{\gamma_{0}}\left\lceil i, \ldots, \eta_{\gamma_{l-1}}\left\lceil i\right.\right.$ for $\gamma_{k}<\alpha$ to $f_{i, l}^{\alpha}$ : each time we obtain a set in the ideal $I_{i}$ and a member $\bar{A}$ of $\prod_{i<\delta} I_{i}$ such that if $\left(\forall^{J} i\right)\left(\eta(i) \notin A_{i}\right)$, 
$\eta \in \prod_{i<\delta} \lambda_{i}$ then $\eta$ satisfies the demand. Eventually we have $|\alpha|^{<\omega}$ such elements of $\prod_{i<\delta} I_{i}$, say $\left\{\bar{B}^{\alpha, \xi}: \xi \leq|\alpha|+\aleph_{0}\right\}$. Then for some $\gamma_{\alpha}$,

$$
\left(\forall \xi<|\alpha|+\aleph_{0}\right)\left(\forall^{J} i<\delta\right)\left(B_{i}^{\alpha, \xi} \subseteq A_{i}^{\gamma_{\alpha}}\right),
$$

and similarly

$$
(\forall \beta<\alpha)\left(\forall^{J} i<\delta\right)\left(\eta_{\beta}(i) \in A_{i}^{\alpha_{i}}\right) .
$$

Choose $\eta_{\alpha} \in \prod_{i<\delta}\left(\lambda_{i} \backslash A_{i}^{\gamma_{\alpha}}\right)$.

REMARK 6.10. One of the main tools used in this section are (variants of) the following observation. Suppose $(\mathbb{B}, \bar{y})$ is a $\lambda$-marked Boolean algebra such that $\mathbb{B}$ is $\theta$-Knaster and if $\varepsilon(\alpha, l)<\lambda$ (for $\alpha<\lambda, l<n$ ) are pairwise distinct then for some $\alpha<\beta<\lambda$, for each $l<n$ we have $\mathbb{B} \models y_{\varepsilon(\alpha, l)} \cap$ $y_{\varepsilon(\beta, l)}=\mathbf{0}$. Then $\left(\theta, \lambda, \lambda^{+}\right) \in \mathcal{K}_{\text {smk }}$.

CONCLUding Remarks 6.11. If $\mu$ is a strong limit singular cardinal and $\operatorname{cf}(\mu)<\theta=\operatorname{cf}(\theta)<\mu$ then, by the methods of [1] or [3], we hope to get consistency of the statement: If an algebra $\mathbb{B}$ satisfies the $\theta$-cc then it satisfies the $\mu^{+}$-Knaster condition.

One may formulate the following question now:

Question (mostly solved) 6.12. Suppose that $\mathbb{B}$ is a Boolean algebra satisfying the $\theta$-cc and $\lambda$ is a regular cardinal between $\mu^{+}$and $\left(2^{\mu}\right)^{+}$. Does $\mathbb{B}$ satisfy the $\lambda$-Knaster condition?

There a reasonable amount of information on consistency of the negative answer in the next section, though 6.12 is not fully answered there. But a real problem is the following.

Problem 6.13. Assume $\lambda=\mu^{+}, \operatorname{cf}(\mu)=\theta$ and $\mu$ is a strong limit singular cardinal. Suppose that an algebra $\mathbb{B}_{0}$ satisfies the $\lambda$-cc and an algebra $\mathbb{B}_{1}$ satisfies the $\theta^{+}$-cc. Does the free product $\mathbb{B}_{0} * \mathbb{B}_{1}$ satisfy the $\lambda$-cc? (Is this consistent? See 5.15.)

Problem 6.14. Is it consistent that each Boolean algebra with the $\aleph_{1^{-}}$ Knaster property has the $\lambda$-Knaster property for every regular (uncountable) cardinal $\lambda$ ?

7. Some consistency results. We had seen that without inner models with large cardinals we have a complete picture, e.g.:

(※) If $\theta=\operatorname{cf}(\theta)>\aleph_{0}, \mathbb{B}$ is a Boolean algebra satisfying the $\theta$-cc and $\lambda$ is a regular cardinal such that

$$
(\forall \tau<\lambda)\left(\tau^{<\theta}<\lambda\right),
$$

then the algebra $\mathbb{B}$ satisfies the $\lambda$-Knaster condition. 
(ב) If $\theta=\operatorname{cf}(\theta)>\aleph_{0}, \theta<\mu=\mu^{<\mu}<\lambda=\operatorname{cf}(\lambda)<\chi=\chi^{\lambda}$, then there is a $\mu^{+}$-cc $\mu$-complete forcing notion $\mathbb{P}$ of size $\chi$ such that

$\Vdash_{\mathbb{P}}$ "the $\theta$-cc implies the $\lambda$-Knaster property".

(I) If $\theta=\operatorname{cf}(\theta)<\mu, \mu$ is a strong limit singular cardinal, $\operatorname{cf}(\mu)=\theta$, then the $\theta^{+}$-cc does not imply the $\mu^{+}$-Knaster property (and even we have a product example).

In (I), if we allow $\left(2^{\theta}\right)$-cc we may even get a better conclusion. In this section we want to show, under a large cardinals hypothesis, the consistency of failure.

Proposition 7.1. Assume that $\kappa$ is a supercompact cardinal, $\kappa<\lambda=$ $\operatorname{cf}(\lambda)$. Let $\mathbb{B}$ be a Boolean algebra which does not have the $\lambda$-Knaster property. Then

$(\exists \theta)\left(\aleph_{0}<\theta=\operatorname{cf}(\theta)<\kappa \& \mathbb{B}\right.$ does not have the $\theta$-Knaster property $)$.

Proof. Since $\kappa$ is supercompact, for every second order formula $\psi$, if $M \models \psi$ then for some $N \prec M,|N|<\kappa, N \models \psi$ (see Kanamori and Magidor $[7])$.

PROPOSITION 7.2. (1) If $\aleph_{0}<\lambda_{0}<\lambda_{1}$ are regular cardinals such that

$(*)_{\lambda_{0}, \lambda_{1}} \quad$ for every $x \in \mathcal{H}\left(\lambda_{1}^{+}\right)$there is $N \prec\left(\mathcal{H}\left(\lambda_{1}^{+}\right), \in\right)$ such that $x \in N$ and $N \cong\left(\mathcal{H}\left(\lambda_{0}^{+}\right), \in\right)$,

then if a Boolean algebra $\mathbb{B}$ has the $\lambda_{0}$-Knaster property then it has the $\lambda_{1}$-Knaster property ( and $\mathbb{B} \models \lambda_{0}$-cc implies $\mathbb{B} \models \lambda_{1}$-cc).

(2) The condition $(*)_{\lambda_{0}, \lambda_{1}}$ above holds if for some $\kappa_{0}, \kappa_{1}, \kappa_{0}<\lambda_{0}, \kappa_{1}<$ $\lambda_{1}$ we have:

$(\oplus)$ there is an elementary embedding $j: \mathbf{V} \rightarrow M$ with the critical point $\kappa_{0}$ and such that $j\left(\kappa_{0}\right)=\kappa_{1}, j\left(\lambda_{0}\right)=\lambda_{1}$ and $M^{\lambda_{1}} \subseteq M$.

(3) If $\kappa_{0}$ is a 2-huge cardinal (or actually less) and, e.g., $\lambda_{0}=\kappa_{0}^{+\omega+1}$ then for some $\lambda_{1}=\kappa_{1}^{+\omega+1}$ the condition $(\oplus)$ above holds (we can assume $G C H)$.

P r o of. Just check.

Proposition 7.3. Assume that

$$
\mathbf{V}=" G C H+\text { there is a 2-huge cardinal }>\theta=\operatorname{cf}(\theta) "
$$

(can think of $\theta=\aleph_{0}$ ). Then there is a $\theta$-complete forcing notion $\mathbb{P}$ such that in $\mathbf{V}^{\mathbb{P}}$ :

(a) GCH holds,

(b) if a Boolean algebra $\mathbb{B}$ has the $\theta^{+}$-Knaster property then it has the $\theta^{+\theta+1}$-Knaster property (note that if $\aleph_{\theta}>\theta$ then $\theta^{+\theta+1}=\aleph_{\theta+1}$ ). 
P r o of. Similar to Levinski, Magidor and Shelah [8].

Chasing arrows what we use is

Proposition 7.4. If $\mathbf{V}=G C H$ (for simplicity), $\theta=\operatorname{cf}(\theta)=\operatorname{cf}(\mu)<\mu$, a Boolean algebra $\mathbb{B}$ does not satisfy the $\mu^{+}-$Knaster condition and $\mathbb{Q}=$ $\operatorname{Levy}(\theta, \mu)$ then $\mathbf{V}^{\mathbb{Q}}=$ "B does not have the $\theta^{+}$-Knaster property".

8. More on getting the Knaster property. Our aim here is to get a ZFC result (under reasonable cardinal arithmetic assumptions) which implies that our looking for $(\kappa$, not $\lambda)$-Knaster marked Boolean algebras near strong limit singular is natural. Below we discuss the relevant background. The proof relies on pcf theory (but only by quoting a simply stated theorem) and seems to be a good example of the applicability of pcf, in particular, for the "revised GCH" of [25].

TheOREM 8.1. Assume $\mu=\mu^{<\beth_{\omega}}$.

(1) If a Boolean algebra $\mathbb{B}$ of cardinality $\leq 2^{\mu}$ satisfies the $\aleph_{1}$-cc then $\mathbb{B}$ is $\mu$-linked (see below).

(2) If $\mathbb{B}$ is a Boolean algebra satisfying the $\aleph_{1}$-cc then $\mathbb{B}$ has the $\lambda$ Knaster property for every regular cardinal $\lambda \in\left(\mu, 2^{\mu}\right]$.

Definition 8.2. (1) A Boolean algebra $\mathbb{B}$ is $\mu$-linked if $\mathbb{B} \backslash\{\mathbf{0}\}$ is the union of $\leq \mu$ sets of pairwise compatible elements.

(2) A Boolean algebra $\mathbb{B}$ is $\mu$-centred if $\mathbb{B} \backslash\{\mathbf{0}\}$ is the union of $\leq \mu$ filters.

Of course we can replace the $\aleph_{1}$-cc, $\beth_{\omega}$ by the $\kappa$-cc, $\beth_{\omega}(\kappa)$ (see more later). The proof is self-contained except a reference to a theorem quoted from $[25]$.

Let us review some background. By $[14,3.1]$, if $\mathbb{B}$ is a $\kappa$-cc Boolean algebra of cardinality $\mu^{+}$and $\mu=\mu^{<\kappa}$ then $\mathbb{B}$ is $\mu$-centred. The proof did not work for $\mathbb{B}$ of cardinality $\mu^{++}$even if $2^{\mu} \geq \mu^{++}$by [16], the point being we consider three elements. But if $\mu=\mu^{<\mu}<\lambda^{<\lambda}$, then for some $\mu^{+}$-cc $\mu$-complete forcing notion $\mathbb{P}$ of cardinality $\lambda$, in $\mathbf{V}^{\mathbb{P}}$ we have:

- if $\mathbb{B}$ is a $\mu$-cc Boolean algebra of cardinality $<\lambda$ then $\mathbb{B}$ is $\mu$-centred

(follows from an appropriate axiom). Hajnal, Juhász and Szentmiklóssy [5] continue this restricting themselves to $\mu$-linked. Then the proof can be carried out for $\mu^{++}$, and they continue by induction. However, as in quite a few cases, the problem was for $\lambda^{+}$when $\operatorname{cf}(\lambda)=\aleph_{0}$, so they assume

$(\otimes) \quad$ if $\quad \lambda \in\left(\mu, 2^{\mu}\right), \operatorname{cf}(\lambda)=\aleph_{0} \quad$ then $\quad \lambda=\lambda^{\aleph_{0}}$ and $\square_{\lambda}$

(on the square, see Jensen [6]). This implies that if we start with $\mathbf{V}=\mathbf{L}$ and force, then the assumption $(\otimes)$ holds, so it is a reasonable assumption. Also they prove the consistency of the failure of the conclusion when $\otimes$ 
fails relying on Hajnal, Juhász and Shelah [4] (on a set system + graph constructed there) and on colouring of graphs (see $[5, \S 2])$. Specifically, they prove the consistency of $2^{\aleph_{0}}=\aleph_{1}, 2^{\aleph_{1}}=\aleph_{\omega+1}$, and for some $\mathbb{B},|\mathbb{B}|=2^{\aleph_{1}}$, $\mathbb{B}$ satisfies the $\aleph_{1}$-cc but is not $\aleph_{1}$-linked, only $\aleph_{2}$-linked.

This gives the impression of essentially closing the issue, and so I would have certainly thought some years ago, but this is not the case, exemplifying the danger of looking at specific cases. In fact, as we shall note in the end, their consistency result is best possible under our knowledge of relevant forcing methods. They use [4] to have "many very disjoint sets" (i.e., $\left\langle X_{\alpha}\right.$ : $\alpha \in S\rangle, S \subseteq\left\{\delta<\aleph_{\omega+1}: \operatorname{cf}(\delta)=\aleph_{1}\right\}, X_{\alpha} \subseteq \alpha=\sup \left(X_{\alpha}\right)$, and $\alpha \neq \beta \Rightarrow$ $X_{\alpha} \cap X_{\beta}$ finite).

On pcf see [22]. Now, [25] has half jokingly a strong claim of proving $\mathrm{GCH}$ under reasonable reinterpretation. In particular [25] says there cannot be many strongly almost disjoint quite large sets, so this blocks reasonable extensions of [5]. Now the main theorem of [25] enables us to carry out the induction on $\lambda \in\left(\mu, 2^{\mu}\right]$ as in $[14,3.1],[5, \S 3]$.

Proposition 8.3. Suppose that:

(a) $\lambda>\theta=\operatorname{cf}(\theta) \geq \kappa=\operatorname{cf}(\kappa)>\aleph_{0}$,

(b) there are a club $E$ of $\lambda$ and a sequence $\overline{\mathcal{P}}=\left\langle\mathcal{P}_{\alpha}: \alpha \in E\right\rangle$ (with $\alpha \in E \Rightarrow|\alpha|$ divides $\alpha)$ such that:

(i) $\mathcal{P}_{\alpha} \subseteq[\alpha]^{<\kappa},\left|\mathcal{P}_{\alpha}\right| \leq|\alpha|$ and $\overline{\mathcal{P}}$ is increasing continuous,

(ii) if $X \subseteq \lambda$ has order type $\theta$, then for some increasing $\left\langle\gamma_{\varepsilon}: \varepsilon<\kappa\right\rangle$ we have $\gamma_{\varepsilon} \in X$ and for each $\varepsilon<\kappa$, for some $\zeta \in(\varepsilon, \kappa)$ and $\alpha \leq \min \left(E \backslash \gamma_{\zeta}\right)$ we have $\left\{\gamma_{\zeta}: \zeta<\varepsilon\right\} \in \mathcal{P}_{\alpha}$,

(c) $\mathbb{B}$ is a Boolean algebra satisfying the $\kappa$-cc, $|\mathbb{B}|=\lambda$.

Then we can find a Boolean algebra $\mathbb{B}^{\prime}$ and a sequence $\left\langle\mathbb{B}_{\alpha}^{\prime}: \alpha \in E\right\rangle$ of subalgebras of $\mathbb{B}^{\prime}$ such that:

$(\alpha) \mathbb{B} \subseteq \mathbb{B}^{\prime} \subseteq \mathbb{B}^{\text {com }}$ (the completion),

$(\beta) \mathbb{B}^{\prime}=\bigcup_{\alpha \in E} \mathbb{B}_{\alpha}^{\prime},\left|\mathbb{B}_{\alpha}^{\prime}\right| \leq|\alpha|+\aleph_{0},\left\langle\mathbb{B}_{\alpha}^{\prime}: \alpha \in E\right\rangle$ is increasing continuous in $\alpha$,

$(\gamma)$ if $\alpha \in E, x \in \mathbb{B}^{\prime} \backslash\{\mathbf{0}\}$ then for some $Y \subseteq \mathbb{B}_{\alpha}^{\prime} \backslash\{\mathbf{0}\}$ with $|Y|<\theta$ we have:

- if $y \in Y$ then $y \cap x=0_{\mathbb{B}^{\prime}}$, and

- if $z \in \mathbb{B}_{\alpha}^{\prime}$ is such that $z \cap x=0_{\mathbb{B}^{\prime}}$ then $z \leq \sup \left(Y^{\prime}\right) \in \mathbb{B}_{\alpha}^{\prime}$ for some $Y^{\prime} \in[Y]^{<\kappa}$,

$(\delta)$ if either $(*)_{1}$ or $(*)_{2}$ below holds then we can add

$Y$ generates the ideal $\left\{z \in \mathbb{B}_{\alpha}^{\prime}: z \cap x=\mathbf{0}_{\mathbb{B}^{\prime}}\right\}$,

where 
$(*)_{1}(\forall \varepsilon<\theta)\left(|\varepsilon|^{<\kappa}<\theta\right)$,

$(*)_{2}$ for some cofinal $\mathcal{P}^{*} \subseteq[\theta]^{<\kappa}$ of cardinality $\theta$, in clause (b) we add: for some unbounded $w \subseteq \theta$, for every $v \in[w]^{<\kappa}$ there is $u$ such that $v \subseteq u \in \mathcal{P}^{*}$ and for $\gamma \in X$ we have $\left\{\gamma_{\varepsilon}: \varepsilon \in u\right\} \in \mathcal{P}_{\gamma}$ (if $\theta=\theta^{<\kappa}$, we can ask $u=v$ ).

Pro of. Let $\chi$ be a large enough regular cardinal. Let $\mathbb{B}=\left\{x_{\varepsilon}: \varepsilon<\lambda\right\}$ and let $\mathbb{B}^{\text {com }}$ be the completion of $\mathbb{B}$. By induction on $\alpha \in E$ we choose an elementary submodel $N_{\alpha}$ of $\left(\mathcal{H}(\chi), \in,<_{\chi}^{*}\right)$ of cardinality $|\alpha|$, increasing continuous in $\alpha$, such that $\mathbb{B},\left\langle x_{\varepsilon}: \varepsilon<\lambda\right\rangle, \mathbb{B}^{\text {com }}, \overline{\mathcal{P}}, \lambda, \theta, \kappa$ belong to $N_{0}$ and $\left\langle N_{\zeta}: \zeta \leq \varepsilon\right\rangle \in N_{\varepsilon+1}$.

Note: if $\alpha \in \operatorname{nacc}(E)$ then $\alpha \in N_{\alpha}$, and hence $\mathcal{P}_{\alpha} \subseteq N_{\alpha}$.

Let

$$
\mathbb{B}_{\alpha}^{\prime}:=N_{\alpha} \cap \mathbb{B}^{\text {com }}, \quad \mathbb{B}^{\prime}=\bigcup_{\alpha \in E} \mathbb{B}_{\alpha}^{\prime} .
$$

By induction on $\alpha \in E$ we define a one-to-one function $g_{\alpha}$ from $\mathbb{B}_{\alpha}^{\prime}$ onto $\alpha$ such that

$$
\beta \in \alpha \cap E \Rightarrow g_{\beta} \subseteq g_{\alpha} \text {, and } g_{\alpha} \text { is the }<_{\chi}^{*} \text {-first such } g,
$$

so $g_{\alpha} \in N_{\min (E \backslash(\alpha+1))}$. Let $g=\bigcup_{\alpha \in E} g_{\alpha}$. Thus $g$ is a one-to-one function from $\mathbb{B}^{\prime}$ onto $\lambda$. Now clearly

(*) if $x \in \mathbb{B}_{\alpha}^{\prime}$ and $\beta=\min \left\{\gamma \in E: g(x) \in \mathbb{B}_{\gamma}^{\prime}\right\}$ then $\beta<\alpha \vee \beta=\alpha$ $\in \operatorname{nacc}(E)$

hence in any case $\beta \in N_{\alpha}$ so $\mathcal{P}_{-\beta} \subseteq N_{\alpha}$.

In the conclusion clauses, $(\alpha),(\beta)$ should be clear; let us prove $(\gamma)$. So let $\alpha \in E$ and $x \in \mathbb{B}^{\prime} \backslash\{\mathbf{0}\}$. We define $J=\left\{z \in \mathbb{B}_{\alpha}^{\prime}: \mathbb{B}^{\prime}=" z \cap x=\mathbf{0}\right.$ " $\}$. Then $J$ is an ideal of $\mathbb{B}_{\alpha}^{\prime}$. We now try to choose by induction on $\varepsilon<\theta$ elements $y_{\varepsilon} \in J$ such that:

(i) $y_{\varepsilon}$ is a member of $J \backslash\left\{\mathbf{0}_{\mathbb{B}}\right\}$,

(ii) there is no $u \in[\varepsilon]^{<\kappa}$ such that $y_{\alpha} \leq \sup _{\zeta \in u} y_{\zeta} \in \mathbb{B}_{\alpha}^{\prime}$ (sup in the complete Boolean algebra $\left.\mathbb{B}^{\text {com }}\right)$,

(iii) under (i) + (ii), $g\left(y_{\varepsilon}\right)(<\lambda)$ is minimal (hence under (i) + (ii), $\beta_{\varepsilon}:=\min \left\{\beta \leq \alpha: y_{\varepsilon} \in \mathbb{B}_{\beta}^{\prime}\right\}$ is minimal).

If we are stuck for some $\varepsilon<\theta$, then for every $y \in J$ the condition (ii) fails (note that (iii) does not change at this point), i.e., there is a corresponding set $u$ so the desired conclusion of $(\gamma)$ holds. So suppose $y_{\varepsilon}$ is defined for $\varepsilon<\theta$. Clearly

$$
\zeta<\varepsilon \Rightarrow g\left(y_{\zeta}\right)<g\left(y_{\varepsilon}\right),
$$

and hence $\zeta<\varepsilon<\theta \Rightarrow \beta_{\zeta} \leq \beta_{\varepsilon}$, and $\zeta<\varepsilon \Rightarrow y_{\zeta} \neq y_{\varepsilon}$. Now apply clause (b)(ii) of the assumption to the set $X=\left\{\gamma_{\varepsilon}^{\prime}: \varepsilon<\theta\right\}$. We get a subset $Y$ of $X$ of order type $\kappa$ such that letting the sequence $\left\langle\gamma_{\varepsilon}: \varepsilon<\kappa\right\rangle$ list $Y$ in increasing order, we have (letting $\gamma_{\varepsilon}=\gamma(\varepsilon)$ ): 
(**) for every $\zeta<\kappa$ for some $\xi \in(\zeta, \kappa)$ the set $\left\{g\left(y_{\gamma_{\varepsilon}}\right): \varepsilon<\zeta\right\}$ belongs to $\mathcal{P}_{\beta_{\gamma(\xi)}}$

[Why? as the $\alpha$ given by clause (b)(ii) is $\min \left(E \backslash g\left(y_{\gamma(\xi)}\right)\right)$ which is $\beta_{\xi}$ by its definition in clause (ii) above, by (*) above the set $\left\{\gamma_{\varepsilon}: \varepsilon<\zeta\right\}$ belongs to $N_{\alpha}$. Also, as in the analysis in $(*), g\left\lceil\left\{y_{\gamma_{\varepsilon}}: \varepsilon<\zeta\right\}\right.$ is included in a one-to-one function from $N_{\alpha}$ hence $\left\{y_{\gamma_{\varepsilon}}: \varepsilon<\zeta\right\}$ belongs to $\left.N_{\alpha}\right]$.

Hence for every $\zeta<\kappa, \sup \left\{y_{\gamma_{\varepsilon}}: \varepsilon<\zeta\right\}$ belongs to $\mathcal{B}_{\alpha}^{\prime}$, but each $y_{\gamma_{\varepsilon}}$ is disjoint to $x$ (in $\mathcal{B}^{\text {com }}$ ) together it belongs to $J$. By our inductive choice of $y_{\gamma}$ for $\gamma<\theta$, we have $y_{\gamma_{\xi}} \not \leq \sup \left\{y_{\gamma_{\varepsilon}}: \varepsilon<\zeta\right\}$. As this holds for every $\zeta<\kappa$ and $\kappa$ is regular we have gotten a contradiction to $\mathcal{B}$, hence $\mathcal{B}^{\text {com }}$ satisfying the $\kappa$-cc, so really clause $(\gamma)$ holds.

We are left with proving clause $(\delta)$ there. We repeat the proof of clause $(\gamma)$, only changing clause (ii) in the inductive choice of $y_{\gamma}$ to

$\left(\right.$ ii) ${ }^{\prime} y_{\varepsilon}$ does not belong to the ideal (of $\mathcal{B}_{\alpha}^{\prime}$ ) generated by $\left\{y_{\zeta}: \zeta<\varepsilon\right\}$.

Again if we are stuck at some $\varepsilon<\theta$ we get the desired conclusion, so assume toward contradiction that $y_{\varepsilon}$ is defined for every $\gamma<\theta$. Now first assume that possibility $(*)_{1}$ from clause $(\delta)$ holds, so clearly for some club $C$ of $\theta$ we have: if $\zeta<\xi \in C$ and $u$ is a subset of $\varepsilon$ of cardinality $<\kappa$ and $\sup \left\{y_{\gamma_{\varepsilon}}: \varepsilon<\zeta\right\}$ belongs to the ideal of $\mathcal{B}_{\alpha}^{\prime}$ generated by $\left\{y_{\gamma_{\varepsilon}}: \varepsilon<\theta\right\}$, then it belongs to the ideal of $\mathcal{B}_{\alpha}^{\prime}$ generated by $\left\{y_{\gamma_{\varepsilon}}: \varepsilon<\xi\right\}$. Now choose an ordinal $\zeta \in \operatorname{acc}(C)$ of cofinality $\kappa$ and continue as in the proof of clause $(\gamma)$.

So clause $(\delta)$ holds when possibility $(*)_{1}$ holds, so assume that possibility $(*)_{2}$ holds. Let $\left\langle u_{\varepsilon}: \varepsilon<\kappa\right\rangle$ list the family $\mathcal{P}^{*}$ of subsets of $\theta$ of cardinality $<\kappa$ each appearing $\kappa$ times. We change the construction by adding to clause (ii):

(ii) ${ }^{+}$if there is $\xi<\theta$ satisfying: $u_{\xi}$ is a subset of $\varepsilon$ and $\sup \left\{y_{\zeta}: \zeta \in u_{\varepsilon}\right\}$ belongs to $\mathcal{B}_{\alpha}^{\prime}$ but does not belong to the ideal of $\mathcal{B}_{\alpha}^{\prime}$ generated by $\left\{y_{\zeta}\right.$ : $\zeta<\varepsilon\}$ then $y_{\varepsilon}$ is equal to such sup for the minimal possible $\xi$.

Note that we probably lose $\zeta<\xi<\theta \Rightarrow \beta_{\zeta} \leq \beta_{\xi}$.

Still, by $(*)_{2}$ applied to $X:=\left\{g\left(y_{\gamma_{\varepsilon}}\right): \varepsilon<\theta\right\}$ we get an unbounded subset $w$ of $\theta$ such that for every $v \in[w]^{<\kappa}$ for some $u \in[w]^{<\kappa}$ and $\varepsilon<\theta$ we have $v \subseteq u$ and $\left\{g\left(y_{\gamma_{\varepsilon}}\right): \varepsilon \in u\right\} \in \mathcal{P}_{\beta_{\varepsilon}}$. Let $v$ be a subset of $w$ of cardinality $<\kappa$ such that $\sup \left\{g\left(y_{\gamma_{\varepsilon}}\right): \varepsilon \in v\right\}$ is equal to $\sup \left\{g\left(y_{\gamma_{\varepsilon}}\right): \varepsilon \in w\right\}$, and let $u \in \mathcal{P}^{*}$ be as guaranteed by $(*)_{2}$. Let $\xi<\theta$ be such that $u_{\xi}=u$, so for every $\varepsilon<\theta$ large enough, $\xi$ satisfies the assumption in (ii) ${ }^{+}$above, but we do not use the same $\xi$ twice, so necessarily for some $\zeta<\theta$ we have $y_{\zeta}=\sup \left(\left\{y_{\gamma}: \gamma \in u_{\xi}\right\}\right.$ but then we can find $\varepsilon \in w \backslash(\zeta+1)$, so $y_{\varepsilon}$ belongs to the ideal generated by $\left\{y_{i}: i<\varepsilon\right\}$, contradiction. 
Proposition 8.4. Suppose that:

(a) $\lambda>\theta=\operatorname{cf}(\theta) \geq \kappa=\operatorname{cf}(\kappa)>\aleph_{0}$ and $\mu=\mu^{<\theta} \leq \lambda \leq 2^{\mu}$

(b) as in assumption (b) of 8.3 and either $(*)_{1}$ or $(*)_{2}$ of clause $(\delta)$ of 8.3 ,

(c) $\mathbb{B}$ is a $\kappa$-cc Boolean algebra of cardinality $\lambda$,

(d) every subalgebra $\mathbb{B}^{\prime} \subseteq \mathbb{B}^{\text {com }}$ of cardinality $<\lambda$ is $\mu$-linked (see Definition $8.2(1))$.

Then $\mathbb{B}$ is $\mu$-linked.

Proof. Let $\left\langle\mathbb{B}_{\alpha}^{\prime}: \alpha \in E\right\rangle, \mathbb{B}$ be as in the conclusion of 8.3. Without loss of generality we may assume that the set of elements $\mathbb{B}_{\alpha}^{\prime}$ is $\alpha$. For $\alpha \in E$, let $h_{\alpha}: \mathbb{B}_{\alpha}^{\prime} \backslash\{\mathbf{0}\} \rightarrow \mu$ be such that

$$
h_{\alpha}\left(x_{1}\right)=h_{\alpha}\left(x_{2}\right) \quad \Rightarrow \quad x_{1} \cap x_{2} \neq \mathbf{0}_{\mathbb{B}} .
$$

For each $x \in \mathbb{B}^{\prime} \backslash \mathbb{B}_{\min (E)}^{\prime}$ let $\alpha(x)=\max \left\{\alpha \in E: x \notin \mathbb{B}_{\alpha}^{\prime}\right\}$ (well defined as $\mathbb{B}^{\prime}=\bigcup_{\alpha \in E} \mathbb{B}_{\alpha}^{\prime}$ and $\left\langle\mathbb{B}_{\alpha}^{\prime}: \alpha \in E\right\rangle$ is increasing continuous), and let $Y_{x, \alpha} \subseteq \mathbb{B}_{\alpha}^{\prime}$ be such that $\left|Y_{x, \alpha}\right|<\theta$ and

$$
Y_{x} \subseteq J_{x}:=\left\{y \in \mathbb{B}_{\alpha}^{\prime}: y \cap x=\mathbf{0}_{\mathbb{B}}\right\}
$$

and $Y_{x}$ is cofinal in $J_{x}$ ( $Y_{x}$ exists by 8.3 , see clause $(\delta)$ ).

Define $u_{x}^{0}=\{0, \alpha(x)\}$, let $Y_{x}^{0}$ be the subalgebra of $\mathbb{B}^{\prime}$ generated by $\{x\}$, and $u_{x}^{n+1}=u_{x}^{n} \cup\left\{\alpha(y): y \in Y_{x}^{n} \backslash \min \left(\mathbb{B}_{\alpha}^{\prime}\right)\right\}$ and $Y_{x}^{n+1}$ be the subalgebra of $\mathbb{B}^{\prime}$ generated by

$$
Y_{x}^{n} \cup \bigcup\left\{Y_{x_{1}, \alpha}: x_{1} \in Y_{x}^{n} \text { and } \alpha \in u_{x}^{n}\right\} .
$$

Finally let $Y_{x}^{\omega}=\bigcup_{n<\omega} Y_{x}^{n}$ and $u_{x}=\bigcup_{n<\omega} u_{x}^{n}$. As $\theta$ is regular, $\left|Y_{x}^{n}\right|<\theta$ and as in addition $\theta$ is uncountable, $\left|Y_{x}^{\omega}\right|<\theta$. Let $u_{x}=\left\{\alpha(y): y \in Y_{x}^{\omega}\right\}$. We can find $A_{\zeta} \subseteq \mathbb{B}^{\prime} \backslash\{\mathbf{0}\}$ for $\zeta<\mu$ such that $\mathbb{B}^{\prime} \backslash\{\mathbf{0}\}=\bigcup_{\zeta<\mu} A_{\zeta}$ and

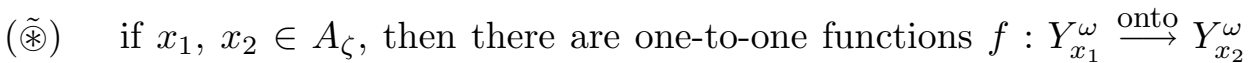
and $g: u_{x_{1}} \stackrel{\text { onto }}{\longrightarrow} u_{x_{2}}$ such that:

(i) $f, g$ preserve the order,

(ii) $f\left(x_{1}\right)=x_{2}$ and if $y \in Y_{x_{1}}^{\omega}$ then $g(\alpha(y))=\alpha(f(y))$,

(iii) if $\alpha \in u_{x_{1}}, y \in \mathbb{B}_{\alpha}^{\prime} \cap Y_{x_{1}}^{\omega}$ then $h_{\alpha}\left(x_{1}\right)=h_{g(\alpha)}\left(f\left(x_{1}\right)\right)$,

(iv) $f$ is an isomorphism (of Boolean algebras),

(v) $g$ is the identity on $u_{x_{1}} \cap u_{x_{2}}$,

(vii) $f$ is the identity on $Y_{x_{1}}^{\omega} \cap Y_{x_{2}}^{\omega}$.

[Why? By [2] or use $\left\langle\eta_{x}: x \in \mathbb{B}^{\prime}\right\rangle, \eta_{x} \in{ }^{\mu} 2$, with no repetitions.]

So it is enough to prove:

$$
x_{1}, x_{2} \in A_{\zeta} \quad \Rightarrow \quad x_{1} \cap x_{2} \neq 0_{\mathbb{B}} .
$$


Let $D_{1}$ be an ultrafilter of $Y_{x_{1}}^{\omega}$ to which $x_{1}$ belongs, and set $D_{2}:=\{f(y)$ : $\left.y \in Y_{x_{2}}^{\omega}\right\}$ (an ultrafilter on $Y_{x_{2}}^{\omega}$ to which $x_{2}$ belongs). It suffices to prove that for each $\alpha \in E$, the set $\left(D_{1} \cap \mathbb{B}_{\alpha}^{\prime}\right) \cup\left(D_{2} \cap \mathbb{B}_{\alpha}^{\prime}\right)$ generates a non-trivial filter on $\mathbb{B}_{\alpha}^{\prime}$. We do it by induction on $\alpha$ (note that if $\alpha \leq \beta$ this holds

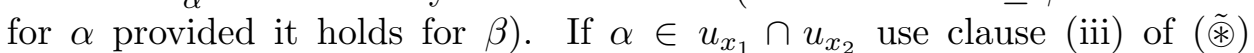
and the choice of $h_{\alpha}$-note that this includes the case when $\alpha=0$. For $\alpha \in \operatorname{acc}(E)$ it follows by the finiteness of the condition. In the remaining case $\beta=\sup (E \cap \alpha)<\alpha$ and if $Y_{x_{1}}^{\omega} \cap \mathbb{B}_{\alpha}^{\prime} \subseteq \mathbb{B}_{\beta}^{\prime}$ and $Y_{x_{2}}^{\omega} \cap \mathbb{B}_{\alpha}^{\prime} \subseteq \mathbb{B}_{\beta}^{\prime}$ this is trivial. So by symmetry we may assume that $\beta \in u_{x_{1}} \backslash u_{x_{2}}$ and use the definition of $Y_{y}$ for $y \in B_{\alpha} \cap Y_{x_{1}}^{\omega} \backslash \mathbb{B}_{\beta}^{\prime}$.

Proposition 8.5. Assume $\mu=\mu^{<\beth_{\omega}(\kappa)}$. Then for every $\lambda \in\left(\mu, 2^{\mu}\right]$ of cardinality $>\mu$, for every large enough regular $\theta<\beth_{\omega}(\kappa)$ clause $(\mathrm{b})$ of 8.3 holds.

Proof. By [25], for every $\tau \in[\mu, \lambda)$ for some $\theta_{\tau}<\beth_{\omega}(\kappa)$, we have:

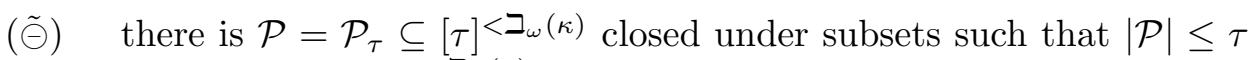
and every $X \in[\tau]<\beth_{\omega}(\kappa)$ is the union of $<\theta_{\tau}$ members of members of $\mathcal{P}_{\tau}$.

Now, as $\operatorname{cf}(\lambda)>\mu$ for some $n<\omega$, the set

$$
\Theta=\left\{\tau: \mu<\tau<\lambda, \theta_{\tau} \leq \beth_{n}(\kappa)\right\}
$$

is an unbounded subset of $\operatorname{Card} \cap(\mu, \lambda)$. Let $\theta<\left(\beth_{n+1}(\kappa)\right)$ be regular. Choose a club $E$ of $\lambda$ such that $\alpha \in \operatorname{nacc}(E) \Rightarrow|\alpha| \in \Theta$, and choose $\mathcal{P}_{\alpha} \subseteq[\alpha]^{<\kappa}$ increasing continuous with $\alpha \in E$ such that for $\alpha \in \operatorname{nacc}(E)$, for every $X \in[\alpha]^{\theta}$, for some $h: X \rightarrow \beth_{n}(\kappa)$, if $Y \subseteq X,|Y|<\kappa$ and $h \nmid Y$ is constant then $Y \in \mathcal{P}_{\alpha}$.

Now suppose $X \subseteq \lambda$, otp $(X)=\theta$, so let $X=\left\{\gamma_{\varepsilon}: \varepsilon<\theta\right\}$ with $\gamma_{\varepsilon}$ increasing with $\varepsilon$; let $\beta_{\varepsilon}=\min \left\{\alpha \in E: \gamma_{\varepsilon}<\beta\right\}$, so $\zeta<\varepsilon \Rightarrow \beta_{\zeta} \leq \beta_{\varepsilon}$ and $\beta_{\varepsilon} \in \operatorname{nacc}(E)$, and there is $h_{\varepsilon}:\{\zeta: \zeta<\varepsilon\} \rightarrow \beth_{n}(\kappa)$ such that for every $j<\beth_{n}(\kappa)$,

$$
u \in[\varepsilon]^{<\kappa} \&\left(h\lceil u \text { constant }) \Rightarrow\left\{\gamma_{\zeta}: \zeta \in u\right\} \in \mathcal{P}_{\beta_{\varepsilon}} .\right.
$$

Applying the Erdös-Rado theorem (i.e., $\left.\theta \rightarrow\left(\beth_{n}(\kappa)^{+}\right)_{\beth_{n}(\kappa)}\right)$ we get the desired result (the proof is an overkill).

Main Conclusion 8.6. Suppose that $\kappa$ is a regular uncountable cardinal, $\mu=\mu^{\beth_{\omega}(\kappa)}$ and $\mathbb{B}$ is a Boolean algebra satisfying the $\kappa$-cc.

(1) If $|\mathbb{B}| \leq 2^{\mu}$ then $\mathbb{B}$ is $\mu$-linked.

(2) If $\lambda$ is regular $\in\left(\mu, 2^{\mu}\right]$ then $\mathbb{B}$ satisfies the $\lambda$-Knaster condition.

Proof. (1) We prove this by induction on $\lambda=|\mathbb{B}|$. If $|\mathbb{B}| \leq \mu$ it is trivial and if cf. $(|\mathbb{B}|) \leq \mu$ it follows easily by the induction hypothesis. In other cases by 8.5 , for some $\theta^{*}<\beth_{\omega}(\kappa)$, for every regular $\theta \in\left(\theta^{*}, \beth_{\omega}(\kappa)\right)$, clause 
(b) of 8.3 holds. Choose $\theta=\left(\theta^{\kappa}\right)^{++}$, so for this $\theta$ both clause (b) of 8.3 and $(*)_{1}$ of clause $(\delta)$ of 8.3 hold. Thus by Proposition 8.4 we can prove the desired conclusion for $\lambda=|\mathbb{B}|$.

(2) Follows from (1).

Proposition 8.7. (1) In 8.6 we can replace the assumption $\mu=\mu^{\beth_{\omega}(\kappa)}$ by $\mu=\mu^{<\tau}$ if

$\otimes \quad$ for every $\lambda \in\left(\mu, 2^{\mu}\right)$ of cardinality $>\mu$, for some $\theta=\operatorname{cf}(\theta) \geq \kappa$ clause (b) of 8.3 and $(*)_{1} \vee(*)_{2}$ of clause $(\delta)$ of 8.3 hold.

(2) If $\lambda^{*} \in\left(\mu, 2^{\lambda}\right)$ and we want to have the conclusion of 8.6(1) with $|\mathbb{B}|=\lambda^{*}$ and 8.6(2) for $\lambda^{*}$-Knaster only then it suffices to restrict ourselves in $\otimes$ to $\lambda \leq \lambda^{*}$.

Proposition 8.8. In 8.3, if $(\forall \varepsilon<\theta)\left[|\varepsilon|^{<\kappa}<\theta\right]$ then we can weaken clause (ii) of assumption (b) to

(ii)' if $X \subseteq \lambda$ has order type $\theta$ then for some $\left\langle\gamma_{\varepsilon}: \varepsilon<\kappa\right\rangle$ we have $\gamma_{\varepsilon} \in X$ and

$$
(\forall \varepsilon<\kappa)(\exists \alpha)\left(\left\{\gamma_{\zeta}: \zeta<\varepsilon\right\} \in \mathcal{P}_{\alpha} \& \alpha=\min \left(E \backslash \sup \left\{\gamma_{\zeta}: \zeta<\varepsilon\right\}\right)\right) .
$$

Proof. Let $X=\left\{j_{\varepsilon}: \varepsilon<\theta\right\}$ be strictly increasing with $\varepsilon$, and let $\beta_{\varepsilon}=\min \left(E \backslash\left(j_{\varepsilon}+1\right)\right)$, so $\zeta<\varepsilon \Rightarrow \beta_{\zeta} \leq \beta_{\varepsilon}$. Let

$e:=\{\varepsilon<\theta: \varepsilon$ is a limit ordinal and

$$
\begin{aligned}
& \text { if } \varepsilon_{1}<\varepsilon \text { and } u \in\left[\varepsilon_{1}\right]^{<\kappa} \text { and }\left\{j_{\xi}: \xi \in u\right\} \in \bigcup_{\zeta<\theta} \mathcal{P}_{\beta_{\zeta}} \\
& \text { then } \left.\left\{j_{\varepsilon}: \varepsilon \in u\right\} \in \bigcup_{\zeta<\varepsilon} \mathcal{P}_{\beta_{\varepsilon}}\right\} .
\end{aligned}
$$

Now, $e$ is a club of $\theta$ as $\left(\theta\right.$ is regular and) $(\forall \varepsilon<\theta)\left[|\varepsilon|^{<\kappa}<\theta\right]$. So we can apply clause (ii) ${ }^{\prime}$ to $X^{\prime}:=\left\{j_{\varepsilon}: \varepsilon \in e\right\}$, and get a subset $\left\{\gamma_{\varepsilon}: \varepsilon<\kappa\right\}$ as there; it is as required in clause (ii).

Proposition 8.9. (1) Assume $\lambda>\theta=\operatorname{cf}(\theta) \geq \kappa=\operatorname{cf}(\kappa)>\aleph_{0}$. Then a sufficient condition for clause $(\mathrm{b})+(\delta)(*)_{1}$ of Claim 8.3 is

$\left(\otimes_{1}\right) \quad($ a) $\lambda>\theta=\operatorname{cf}(\theta)$,

(b) for arbitrarily large $\alpha<\lambda$ for some regular $\tau<\theta$ and $\lambda^{\prime} \leq \lambda$, for every $\mathfrak{a} \subseteq \operatorname{Reg} \cap|\alpha| \backslash \theta$ of cardinality $\leq \theta$ for some $\left\langle\mathfrak{b}_{\varepsilon}: \varepsilon<\right.$ $\left.\varepsilon^{*}<\tau\right\rangle$ we have $\mathfrak{a}=\bigcup_{\varepsilon<\varepsilon^{*}} \mathfrak{b}_{\varepsilon}$ and $\left[\mathfrak{b}_{\varepsilon}\right]^{<\kappa} \subseteq J_{\leq \lambda^{\prime}}[\mathfrak{a}]$ for every $\varepsilon<\varepsilon^{*}$

(c) $(\forall \varepsilon<\theta)\left[|\varepsilon|^{<\kappa}<\theta\right]$ or for every $\lambda^{\prime} \in[\mu, \lambda], \square_{\left\{\delta<\lambda^{\prime}: \mathrm{cf}(\delta)=\theta\right\}}$.

(2) Assume $\mu>\theta \geq \kappa=\operatorname{cf}(\kappa)>\aleph_{0}$. A sufficient condition for clause (b) of 8.3 to hold is:

- for every $\lambda \in\left[\mu, 2^{\mu}\right]$ of cofinality $>\mu$, for some $\theta^{\prime} \leq \theta,\left(\otimes_{1}\right)$ holds (with $\theta^{\prime}$ instead $\theta$ ).

Proof. (1) By [23], [18, 2.6], or [13]. (2) Should be clear. 
REMARK 8.10. So it is still possible that (assuming $\mathrm{CH}$ for simplicity) $\otimes \quad$ if $\mu=\mu^{\aleph_{1}}, \mathbb{B}$ is a c.c.c. Boolean algebra, $|\mathbb{B}| \leq 2^{\mu}$ then $\mathbb{B}$ is $\mu$-linked.

On the required assumption see [19, Hyp. 6.1(x)].

Note that the assumptions of the form $\lambda \in I[\lambda]$ if added save us a little on pcf hyp. (we mention it in 6.5). But if we are interested in $[\kappa$-cc $\Rightarrow \lambda$ Knaster], it can be waived.

\section{References}

[1] M. Džamonja and S. Shelah, Universal graphs at successors of singular strong limits.

[2] R. Engelking and M. Karłowicz, Some theorems of set theory and their topological consequences, Fund. Math. 57 (1965), 275-285.

[3] M. Gitik and S. Shelah, On densities of free products, Topology Appl. 88 (1998), 219-238.

[4] A. Hajnal, I. Juhász and S. Shelah, Splitting strongly almost disjoint families, Trans. Amer. Math. Soc. 295 (1986), 369-387.

[5] A. Hajnal, I. Juhász and Z. Szentmiklóssy, On the structure of CCC partial orders, Algebra Universalis, to appear.

[6] R. B. Jensen, The fine structure of the constructible hierarchy, Ann. Math. Logic 4 (1972), 229-308.

[7] A. Kanamori and M. Magidor, The evolution of large cardinal axioms in set theory, in: Higher Set Theory, Lecture Notes in Math. 669, Springer, 1978, 99-275.

[8] J. P. Levinski, M. Magidor and S. Shelah, Chang's conjecture for $\aleph_{\omega}$, Israel J. Math. 69 (1990), 161-172.

[9] M. Magidor and S. Shelah, When does almost free imply free? (For groups, transversal etc.), J. Amer. Math. Soc. 7 (1994), 769-830.

[10] D. Monk, Cardinal Invariants of Boolean Algebras, Lectures in Mathematics, ETH Zurich, Birkhäuser, Basel, 1990.

[11] —, Cardinal Invariants of Boolean Algebras, Progr. Math., Birkhäuser, Basel, 1996.

[12] S. Shelah, Categoricity of an abstract elementary class in two successive cardinals, Israel J. Math. accepted;. math.LO/9805146 $\left({ }^{1}\right)$.

[13] —, PCF and infinite free subsets, Arch. Math. Logic, accepted; math.LO/9807177.

[14] -, Remarks on Boolean algebras, Algebra Universalis 11 (1980), 77-89.

[15] —, Simple unstable theories, Ann. Math. Logic 19 (1980), 177-203.

[16] -, On saturation for a predicate, Notre Dame J. Formal Logic 22 (1981), 239-248.

[17] - Products of regular cardinals and cardinal invariants of products of Boolean algebras, Israel J. Math. 70 (1990), 129-187.

[18] -, Advances in Cardinal Arithmetic, in: Finite and Infinite Combinatorics in Sets and Logic, N. W. Sauer et al. (eds.), Kluwer, 1993, 355-383.

[19] —, More on cardinal arithmetic, Arch. Math. Logic 32 (1993), 399-428.

[20] -, $\aleph_{\omega+1}$ has a Jonsson algebra, Chapter II of [20].

[21] - Basic: Cofinalities of small reduced products, Chapter I of [20].

[22] —, Cardinal Arithmetic, Oxford Logic Guides 29, Oxford Univ. Press, 1994.

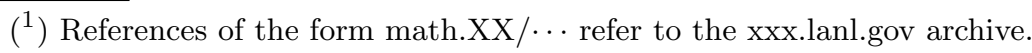


[23] S. Shelah, Further cardinal arithmetic, Israel J. Math. 95 (1996), 61-114; math. $\mathrm{LO} / 9610226$.

[24] -, Colouring and non-productivity of $\aleph_{2}-c c$, Ann. Pure Appl. Logic 84 (1997), 153-174; math.LO/9609218.

[25] - , The Generalized Continuum Hypothesis revisited, Israel J. Math. 116 (2000), 285-321; math.LO/9809200.

[26] R. M. Solovay, Strongly compact cardinals and the GCH, in: Proc. Tarski Symposium (Berkeley, 1971), Proc. Sympos. Pure Math. 25, Amer. Math. Soc., 1974, $365-372$.

Institute of Mathematics

The Hebrew University of Jerusalem

91904 Jerusalem, Israel

E-mail: shelah@math.huji.ac.il
Department of Mathematics Rutgers University

New Brunswick, NJ 08854, U.S.A. URL: http://www.math.rutgers.edu/ shelah

Received 2 September 1996;

in revised form 9 August 1999 